\title{
Modular Synthesis of Cyclic Peptidomimetics Inspired by ЪTurns
}

Senthil Kumar Ramanathan, John Keeler, Huey-Lih Lee, D. Srinivasa Reddy, Gerald Lushington, and Jeffrey Aube*

Department of Medicinal Chemistry, 1251 Wescoe Hall Drive, Malott Hall, Room 4070, University of Kansas, Lawrence, KS 66045-7582, USA and the KU Chemical Methodologies and Library Development Center of Excellence, University of Kansas, 1501 Wakarusa Drive, Lawrence, $K S$, 66047.

\author{
Supporting Information
}

\author{
Experimental Details
}

Copies of ${ }^{1} \mathrm{H}$ - and ${ }^{13} \mathrm{C}-\mathrm{NMR}$ Spectra 


\section{Experimental Details}

General Procedure for Reduction of Amino Acids. ${ }^{1}$ Phenylalaninol. To a cold solution of $\mathrm{LiBH}_{4}(1.31 \mathrm{~g}, 60.5 \mathrm{mmol}, 2$ equiv) in $40 \mathrm{~mL}$ of THF was added trimethyl silyl chloride (15.3 mL, $60.5 \mathrm{mmol}, 2$ equiv). The ice/water bath was removed and the mixture allowed to stir at room temperature for $20 \mathrm{~min}$. Then the mixture was recooled to $0{ }^{\circ} \mathrm{C}$ and phenylalanine (5 g, $30.2 \mathrm{mmol}, 1$ equiv) was added and the reaction mixture was stirred for $12 \mathrm{~h}$. The reaction mixture was quenched with methanol and the reaction mixture was concentrated. The residue was partitioned between 2.5 M aqueous sodium hydroxide solution and methylene chloride, washed with brine, and dried over anhydrous $\mathrm{Na}_{2} \mathrm{SO}_{4}$. The organic layers were filtered and concentrated to afford an oil that was used without further purification.

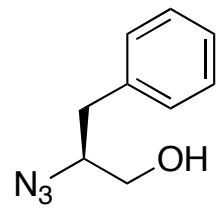

\section{General Procedure for Azidation of Amino Alcohols. ${ }^{2}$}

(S)-2-Azido-3-phenylpropan-1-ol (1c). $\mathrm{NaN}_{3}$ (11.2 g, $172 \mathrm{mmol}, 4.9$ equiv) was dissolved in $35 \mathrm{~mL}$ of water and cooled to $0{ }^{\circ} \mathrm{C} . \mathrm{CH}_{2} \mathrm{Cl}_{2}(40 \mathrm{~mL})$ was added to the vigorously stirred solution followed by trifluromethanesulfonic anhydride $(10.0 \mathrm{~g}, 5.96 \mathrm{~mL}, 35.4 \mathrm{mmol})$. The reaction mixture was allowed to stir for $2 \mathrm{~h}$ at $0{ }^{\circ} \mathrm{C}$. The organic phase was separated and the aqueous layer extracted with methylene chloride $(2 \square 10 \mathrm{~mL})$. The combined organic layers were washed with sodium bicarbonate solution and water and dried over anhydrous sodium sulfate. The yield was taken to be $50 \%$ for the purpose of determining the amounts of triflyl azide used in subsequent reactions (base titration of triflic acid formed during reaction indicated that about $50 \%$ of the anhydride had hydrolyzed during the reaction). To a solution of phenylalaninol (1.41 g, $9.2 \mathrm{mmol}, 1$ equiv) in methylene chloride $(5 \mathrm{~mL})$ was added 4-(dimethylamino)pyridine (4.96 g, 40.6 mmol, 4.4 equiv) at room temperature. Subsequently triflyl azide was added dropwise. The reaction mixture was allowed to stir at room temperature for $2 \mathrm{~h}$. The reaction mixture was concentrated and the known azido alcohol 1c was isolated using flash chromatography. Colorless oil; yield 93\%; $R_{f} 0.66$ (1:1, EtOAc/hexane); [ $\left.\square\right]_{\mathrm{D}} \square 2.4$ (c 1.0, $\mathrm{CHCl}_{3}$ ). IR (neat): 3375, $2105 \mathrm{~cm}^{-1}$. ${ }^{1} \mathrm{H}$ NMR (400 MHz, $\mathrm{CDCl}_{3}$ ): 2.94-2.83 (m, 2H); 3.2 (br s, 1H); 3.62-3.57 (dd, $J=$ 7.8, $12.2 \mathrm{~Hz}, 1 \mathrm{H}) ; 3.78-3.73(\mathrm{t}, J=8.4 \mathrm{~Hz}, 2 \mathrm{H}) ; 7.41-7.29(\mathrm{~m}, 5 \mathrm{H}) .{ }^{13} \mathrm{C} \mathrm{NMR}(100.6 \mathrm{MHz}$, $\left.\mathrm{CDCl}_{3}\right): 37.4,64.8,65.7,127.6,128.9,129.8,137.6$. 


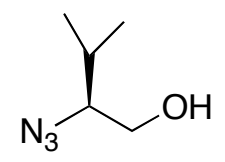

(S)-2-Azido-3-methylbutan-1-ol (1a). Colorless oil; yield 90\%; $R_{f} 0.86$ (1:1,

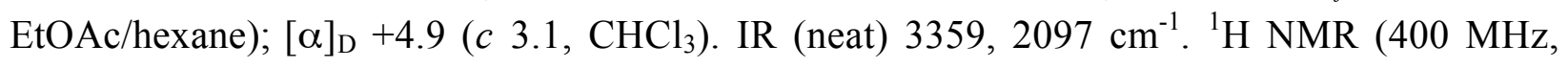
$\mathrm{CDCl}_{3}$ ): $0.95(\mathrm{~d}, J=3.4 \mathrm{~Hz}, 3 \mathrm{H}) ; 0.97$ (d, $\left.J=3.7 \mathrm{~Hz}, 3 \mathrm{H}\right) ; 1.87-1.76$ (septet, $\left.J=6.6 \mathrm{~Hz}, 1 \mathrm{H}\right)$; 2.87 (br s, $1 \mathrm{H}) ; 3.27-3.24(\mathrm{~m}, 1 \mathrm{H}) ; 3.62-3.56(\mathrm{~m}, 1 \mathrm{H}) ; 3.75-3.71(\mathrm{~m}, 1 \mathrm{H}) .{ }^{13} \mathrm{C} \mathrm{NMR}(100.6 \mathrm{MHz}$, $\mathrm{CDCl}_{3}$ ): 18.8, 19.9, 30.2, 63.9, 71.1; MS (EI) calcd for $\mathrm{C}_{5} \mathrm{H}_{11} \mathrm{~N}_{3} \mathrm{O}$ 129.0; found, m/e 129.0.

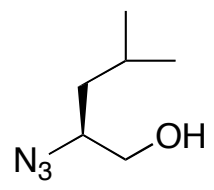

(S)-2-Azido-4-methylpentan-1-ol (1b). Colorless oil; yield 86\%; $R_{f} 0.83$ (1:1,

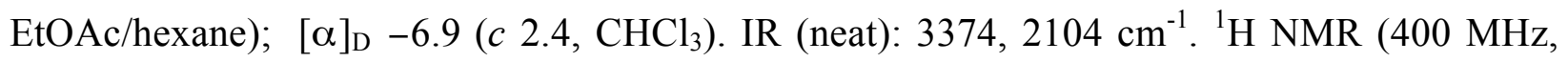
$\left.\mathrm{CDCl}_{3}\right): 0.92(\mathrm{~d}, J=1.2 \mathrm{~Hz}, 3 \mathrm{H}) ; 0.94(\mathrm{~d}, J=1.3 \mathrm{~Hz}, 3 \mathrm{H}) ; 1.29-1.22(\mathrm{~m}, 1 \mathrm{H}) ; 1.45-1.38$ (m, $1 \mathrm{H}$ ); 1.77-1.74 (septet, $J=1.8 \mathrm{~Hz}, 1 \mathrm{H}) ; 2.95$ (br s, $1 \mathrm{H}) ; 3.52-3.46$ (m, 2H); 4.11-4.04 (m, 1H). ${ }^{13} \mathrm{C}$ NMR $\left(100.6 \mathrm{MHz}, \mathrm{CDCl}_{3}\right): 22.3,23.4,25.3,39.7,63.9,65.9$; MS (EI) calcd for $\mathrm{C}_{6} \mathrm{H}_{13} \mathrm{~N}_{3} \mathrm{O}$ 143.10; found, $m / e$ 143.0.

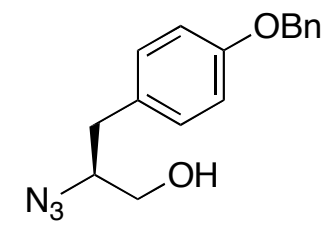

(S)-2-Azido-3-(4-(benzyloxy)phenyl)propan-1-ol (1d). White solid; yield 87\%; mp

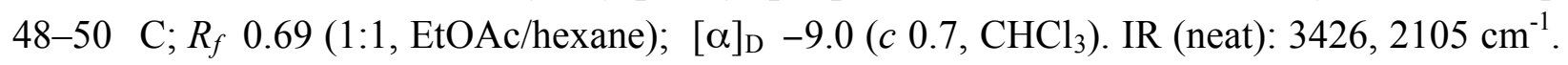
${ }^{1} \mathrm{H}$ NMR (400 MHz, $\left.\mathrm{CDCl}_{3}\right): 2.46(\mathrm{~s}, 1 \mathrm{H}) ; 2.88-2.77(\mathrm{~m}, 2 \mathrm{H}) ; 3.57$ (br s, $\left.1 \mathrm{H}\right) ; 3.74-3.66(\mathrm{~m}, 2 \mathrm{H})$; $5.09(\mathrm{~s}, 2 \mathrm{H}) ; 6.97(\mathrm{~d}, J=8.3 \mathrm{~Hz}, 2 \mathrm{H}) ; 7.17(\mathrm{~d}, J=8.3 \mathrm{~Hz}, 2 \mathrm{H}), 7.49-7.38(\mathrm{~m}, 5 \mathrm{H}) .{ }^{13} \mathrm{C}$ NMR (100.6 MHz, $\left.\mathrm{CDCl}_{3}\right)$ : 40.3, 54.7, 66.6, 70.5, 115.4, 127.9, 128.4, 129.0, 130.6, 131.4, 137.5, 157.9. HRMS calcd for $\mathrm{C}_{16} \mathrm{H}_{17} \mathrm{~N}_{3} \mathrm{O}_{2}\left(\mathrm{M}^{+}+\mathrm{H}\right), 283.1321$; found, 283.1319 . 


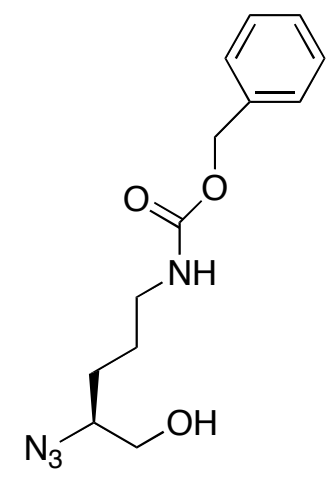

(S)-Benzyl-4-azido-5-hydroxypentylcarbamate (1e). Colorless oil; yield 71\%; $R_{f} 0.64$

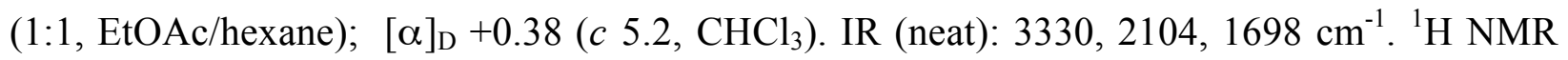
$\left(400 \mathrm{MHz}, \mathrm{CDCl}_{3}\right): 1.59-1.43(\mathrm{~m}, 4 \mathrm{H}) ; 3.16(\mathrm{~d}, J=5.1 \mathrm{~Hz}, 2 \mathrm{H}) ; 3.28(\mathrm{~s}, 1 \mathrm{H}) ; 3.55(\mathrm{~d}, J=7.1$ $\mathrm{Hz}, 1 \mathrm{H}) ; 3.67(\mathrm{~d}, J=2.5 \mathrm{~Hz}, 1 \mathrm{H}) ; 4.15(\mathrm{~s}, 1 \mathrm{H}) ; 5.07(\mathrm{~s}, 2 \mathrm{H}) ; 5.48-5.24(\mathrm{~m}, 1 \mathrm{H}) ; 7.33(\mathrm{~s}, 5 \mathrm{H}) .{ }^{13} \mathrm{C}$ NMR (100.6 MHz, $\left.\mathrm{CDCl}_{3}\right)$ : 26.8, 28.0, 41.0, 63.3, 65.3, 66.9, 128.4, 128.7, 129.0, 136.9, 157.3. HRMS calcd for $\mathrm{C}_{13} \mathrm{H}_{19} \mathrm{~N}_{4} \mathrm{O}_{3}\left(\mathrm{M}^{+}+\mathrm{H}\right), 279.1457$; found, 279.1458 .

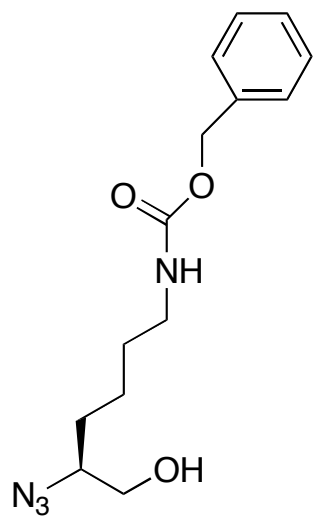

(S)-Benzyl-5-azido-6-hydroxyhexylcarbamate (1f). Colorless oil; yield 93\%; $R_{f} 0.60$

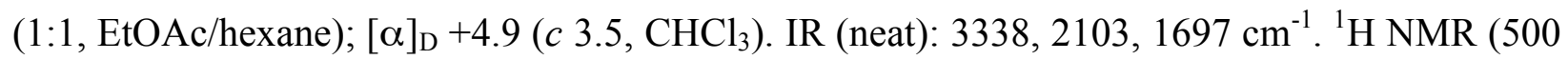
$\left.\mathrm{MHz}_{\mathrm{CDCl}}\right): 1.50-1.28(\mathrm{~m}, 6 \mathrm{H}) ; 3.18(\mathrm{~s}, 2 \mathrm{H}) ; 3.44(\mathrm{~s}, 1 \mathrm{H}) ; 3.55$ (d, J= $\left.7 \mathrm{~Hz}, 1 \mathrm{H}\right) ; 3.67$ (d, $J=$ $8.6 \mathrm{~Hz}, 1 \mathrm{H}) ; 3.8$ (br s, $1 \mathrm{H}) ; 5.09(\mathrm{~s}, 2 \mathrm{H}) ; 5.17$ (s, 1H); 7.35 (s, 5H). ${ }^{13} \mathrm{C} \mathrm{NMR}(125.75 \mathrm{MHz}$, $\left.\mathrm{CDCl}_{3}\right): 22.9,29.6,29.9,40.5,63.9,64.8,66.9,128.0,128.08,128.47,136.38,156.6$. HRMS calcd for $\mathrm{C}_{14} \mathrm{H}_{21} \mathrm{~N}_{4} \mathrm{O}_{3}\left(\mathrm{M}^{+}+\mathrm{H}\right)$ : 293.1614; found, 293.1613 . 


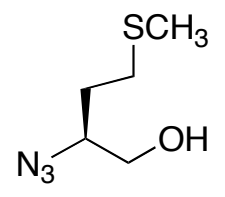

(S)-2-Azido-4-(methylthio)butan-1-ol (1g). Colorless oil; yield 82\%; $R_{f} 0.74$ (1:1, EtOAc/hexane); [0] $\square 30$ (c 2.9, $\mathrm{CHCl}_{3}$ ). IR (neat): 3392, $2123 \mathrm{~cm}^{-1} .{ }^{1} \mathrm{H}$ NMR $(500 \mathrm{MHz}$, $\left.\mathrm{CDCl}_{3}\right): 1.61-1.54(\mathrm{~m}, 2 \mathrm{H}) ; 1.93(\mathrm{~s}, 3 \mathrm{H}) ; 2.49-2.36(\mathrm{~m}, 2 \mathrm{H}) ; 3.46-3.40(\mathrm{~m}, 2 \mathrm{H}) ; 3.54$ (dd, $J=$ 2.6, $10.2 \mathrm{~Hz}, 1 \mathrm{H}$ ); 3.79 (br s, $1 \mathrm{H}) .{ }^{13} \mathrm{C}$ NMR $\left(125.7 \mathrm{MHz}, \mathrm{CDCl}_{3}\right): 15.1,29.5,30.2,62.6,64.8$. HRMS calcd for $\mathrm{C}_{5} \mathrm{H}_{12} \mathrm{~N}_{3} \mathrm{O}_{1} \mathrm{~S}_{1}\left(\mathrm{M}^{+}+\mathrm{H}\right): 162.0701$; found, 162.0676 .

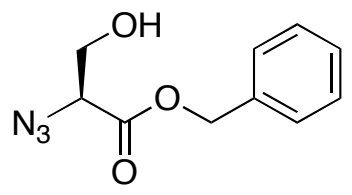

(S)-Benzyl-2-azido-3-hydroxypropanoate (1h). Colorless oil; yield 90\%; $R_{f} 0.81$ (1:1 EtOAc/hexane); [ $\square]_{\mathrm{D}} \square 26$ (c 1.4, $\mathrm{CHCl}_{3}$ ). IR (neat): 3435, 2112, $1743 \mathrm{~cm}^{-1} .{ }^{1} \mathrm{H}$ NMR $(500 \mathrm{MHz}$, $\left.\mathrm{CDCl}_{3}\right): 2.83$ (br s, $\left.1 \mathrm{H}\right) ; 3.94(\mathrm{~d}, J=4.5 \mathrm{~Hz}, 2 \mathrm{H}) ; 4.11(\mathrm{t}, J=4.5 \mathrm{~Hz}, 1 \mathrm{H}) ; 5.31(\mathrm{~s}, 2 \mathrm{H}) ; 7.40-$ $7.30(\mathrm{~m}, 5 \mathrm{H}) .{ }^{13} \mathrm{C}$ NMR $\left(125.75 \mathrm{MHz}, \mathrm{CDCl}_{3}\right): 62.6,63.4,67.7,127.0,128.3,128.6,134.8$, 164.7. HRMS calcd for $\mathrm{C}_{14} \mathrm{H}_{21} \mathrm{~N}_{4} \mathrm{O}_{3}\left(\mathrm{M}^{+}+\mathrm{NH}_{4}\right)$ : 239.1144; found, 239.1146 .

Ketones 2a-2c. Compounds $2 \mathrm{a}$ and $2 \mathrm{c}$ are commercially available from Aldrich. Compound $\mathbf{2 b}$ was synthesized according to literature procedure. ${ }^{3}$

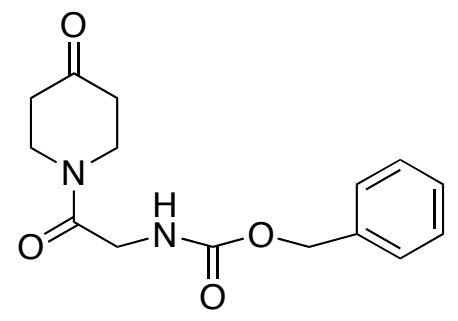

Benzyl-2-oxo-2-(4'-oxopiperidin-1-yl)ethylcarbamate (2d). To a stirred solution of 4piperidone hydrochloride $(5 \mathrm{~g}, 32.5 \mathrm{mmol})$ in THF $(50 \mathrm{~mL})$ was added $\mathrm{Et}_{3} \mathrm{~N}(18.2 \mathrm{~mL}, 130$ $\mathrm{mmol})$. The solution was transferred to a flask containing 2-benzyloxycarbonylacetic acid (8.17 g, $39.1 \mathrm{mmol})$, DCC (10.14 g, $49 \mathrm{mmol})$, and HOBt $(6.59 \mathrm{~g}, 48 \mathrm{mmol})$. After $12 \mathrm{~h}$ the reaction mixture was diluted with ethyl acetate $(250 \mathrm{~mL})$, filtered to remove dicylohexylurea, and concentrated. Ethyl acetate $(250 \mathrm{~mL})$ was added to the residue, which was then washed with water, saturated aqueous sodium bicarbonate, $1 \mathrm{~N} \mathrm{HCl}$, and brine. The organic layer was dried 
over anhydrous sodium sulfate and concentrated in vacuo. Column chromatography afforded the title compound. White solid; mp $115-117^{\circ} \mathrm{C}$, yield 72\%; $\mathrm{R}_{\mathrm{f}} 0.5$ (EtOAc, 100\%); IR (neat): 1718, 1642, $1425 \mathrm{~cm}^{-1} .{ }^{1} \mathrm{H}$ NMR (500 MHz, $\left.\mathrm{CDCl}_{3}\right): 2.51$ (s, 4H); 3.71 (s, 2H), 3.92 (s, 2H), 4.14 (s, $2 \mathrm{H}) ; 5.15(\mathrm{~s}, 2 \mathrm{H}) ; 5.80(\mathrm{~s}, 1 \mathrm{H}), 7.74-7.28(\mathrm{~m}, 5 \mathrm{H}) .{ }^{13} \mathrm{C} \mathrm{NMR}\left(125.75 \mathrm{MHz}, \mathrm{CDCl}_{3}\right): 40.5,40.6$, $41.0,42.5,42.7,66.9,127.9,128.1,128.4,136.2,156.1,166.8,205.6$. HRMS calcd for $\mathrm{C}_{15} \mathrm{H}_{19} \mathrm{~N}_{2} \mathrm{O}_{4}\left(\mathrm{M}^{+}+\mathrm{H}\right): 291.1345$; found, 291.1365 .

General Procedure for the Syntheses of 1,4-Diazepin-5-ones. To a solution of $\mathrm{N}$ benzyl-4-piperidone $(0.100 \mathrm{~g}, 0.52 \mathrm{mmol})$ and azido alcohol (1) -h; $0.15 \mathrm{~g}, 0.84 \mathrm{mmol})$ in $\mathrm{CH}_{2} \mathrm{Cl}_{2}(2 \mathrm{~mL})$ was added trifluromethanesulfonic acid $(0.39 \mathrm{~g}, 0.23 \mathrm{~mL}, 2.64 \mathrm{mmol})$ dropwise at $0{ }^{\circ} \mathrm{C}$. The solution was allowed to warm to room temperature and stirred for $12 \mathrm{~h}$. The solution was concentrated under reduced pressure and a saturated solution of $\mathrm{NaHCO}_{3}(20 \mathrm{~mL})$ was added. The reaction was stirred for an additional $12 \mathrm{~h}$ at room temperature whereupon $25 \mathrm{~mL}$ of $\mathrm{CH}_{2} \mathrm{Cl}_{2}$ was added. The organic layers were washed with water, dried over anhydrous $\mathrm{Na}_{2} \mathrm{SO}_{4}$, and concentrated to give a crude yellow oil. Column chromatography afforded the desired compounds.

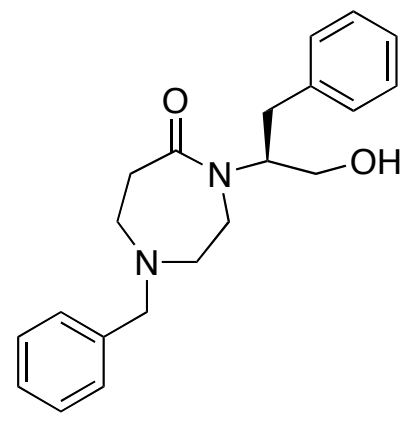

(S)-1-Benzyl-4-(1'-hydroxy-3'-phenylpropan-2'-yl)-1,4-diazepan-5-one (3a). Colorless oil; yield 84\%; $R_{f} 0.13(\mathrm{MeOH} / \text { EtOAc, 1:9); [ } \square]_{\mathrm{D}} \square 40$ (c 4.5, $\left.\mathrm{CHCl}_{3}\right)$. IR (neat): 3380, $1623 \mathrm{~cm}^{-1} .{ }^{1} \mathrm{H}$ NMR (400 MHz, $\left.\mathrm{CDCl}_{3}\right): 2.35$ (s, 2H); 2.49 (d, J=5.1 Hz, 2H); 2.58 (s, 2H); 2.91-2.80 (m, 2H); $3.31(\mathrm{~d}, J=3.9 \mathrm{~Hz}, 2 \mathrm{H}) ; 3.44(\mathrm{~s}, 2 \mathrm{H}) ; 3.94-3.63(\mathrm{~m}, 2 \mathrm{H}) ; 4.25$ (br s, $1 \mathrm{H})$; $4.72(\mathrm{~s}, 1 \mathrm{H}) ; 7.31-7.19(\mathrm{~m}, 10 \mathrm{H}) .{ }^{13} \mathrm{C} \mathrm{NMR}\left(100.6 \mathrm{MHz}, \mathrm{CDCl}_{3}\right)$ : 35.3, 39.0, 46.4, 50.9, 56.1, 59.5, 63.2, 63.3, 126.9, 127.9, 128.8, 128.9, 129.2, 129.4, 138.4, 138.6, 176.7. HRMS calcd for $\mathrm{C}_{21} \mathrm{H}_{27} \mathrm{~N}_{2} \mathrm{O}_{2}\left(\mathrm{M}^{+}+\mathrm{H}\right): 339.2073$; found, 339.2058. 


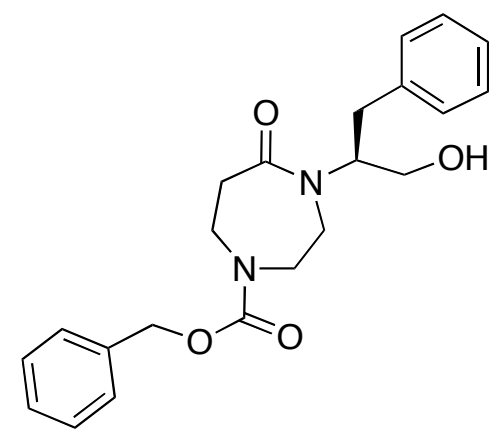

\section{(S)-1-Benzyl-4-(1'-hydroxy-3'-phenylpropan-2'-yl)-5-oxo-1,4-diazepan-1-}

carboxylate (3b). Colorless oil; yield 46\%; $R_{f} 0.13(\mathrm{MeOH} / \mathrm{EtOAc}, 1: 9) ;[\square]_{\mathrm{D}}[3.7$ (c 0.9, $\mathrm{CHCl}_{3}$ ). IR (neat): 3400, $1644 \mathrm{~cm}^{-1} .{ }^{1} \mathrm{H}$ NMR (400 MHz, $\left.\mathrm{CDCl}_{3}\right): 2.57$ (m, 2H); 2.88 (m, 2H); 3.75-3.29 (complex, 9H); 4.73 (br s, 1H); 5.11 (s, 2H); 7.36-7.18 (m, 10H). ${ }^{13} \mathrm{C}$ NMR (100.6 $\left.\mathrm{MHz}, \mathrm{CDCl}_{3}\right):$ 21.5, 35.1, 40.0, 42.1, 47.3, 59.8, 63.5, 67.9, 128.4, 128.8, 128.9, 129.2, 129.6, 136.7, 138.2, 155.7, 176.0. HRMS calcd for $\mathrm{C}_{22} \mathrm{H}_{27} \mathrm{~N}_{2} \mathrm{O}_{4}\left(\mathrm{M}^{+}+\mathrm{H}\right)$ : 383.1971; found, 383.1958.

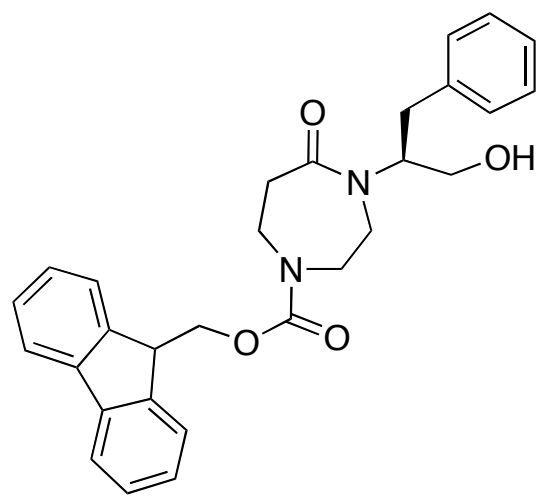

\section{(S)-[9H-Fluoren-9马yl]methyl-4-[1Ghydroxy-3Gphenylpropan-2马yl]-5-oxo-1,4-}

diazepane-1-carboxylate (3c). White foam; yield 74\%; $R_{f} 0.13(\mathrm{MeOH} / \mathrm{EtOAc}, 1: 9) ;[\square]_{\mathrm{D}} \square 2.8$ (c 1, $\mathrm{CHCl}_{3}$ ). IR (neat): 3416, 1700, $1629 \mathrm{~cm}^{-1} .{ }^{1} \mathrm{H}$ NMR (400 MHz, $\left.\mathrm{CDCl}_{3}\right): 2.32$ (m, 1H); 2.46 $(\mathrm{m}, 1 \mathrm{H}) ; 2.88(\mathrm{~d}, J=8.3 \mathrm{~Hz}, 2 \mathrm{H}) ; 3.34-2.87$ (complex, 9H); $4.73(\mathrm{~m}, 1 \mathrm{H}) ; 5.08$ (s, 2H); 7.367.15 (m, 10H). ${ }^{13} \mathrm{C}$ NMR (100.6 MHz, $\left.\mathrm{CDCl}_{3}\right)$ : 35.0, 39.9, 42.1, 47.4, 47.8, 64.0, 67.6, 120.4, 125.2, 127.2, 127.5, 128.1, 129.1, 129.3, 138.2, 141.8, 144.2, 175.8 HRMS calcd for $\mathrm{C}_{29} \mathrm{H}_{31} \mathrm{~N}_{2} \mathrm{O}_{4}$ $\left(\mathrm{M}^{+}+\mathrm{H}\right):$ 471.2284; found, 471.2276 . 


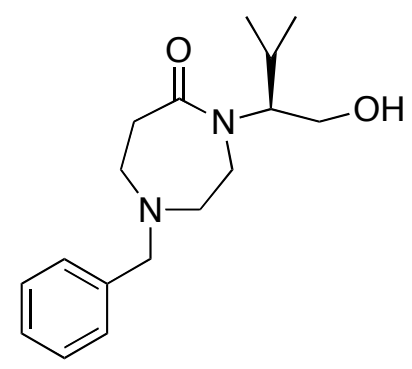

(S)-1-Benzyl-4-(1'-hydroxy-3'-methylbutan-2'-yl)-1,4-diazepan-5-one (4). Yellow oil; yield 89\%; $R_{f} 0.9\left(\mathrm{CH}_{2} \mathrm{Cl}_{2}: \mathrm{MeOH}, 9: 1\right)$; [0]D $\square 13.6$ (c 2.1, $\left.\mathrm{CHCl}_{3}\right)$. IR (neat): 3394, $1627 \mathrm{~cm}^{-1}$. ${ }^{1} \mathrm{H}$ NMR $\left(400 \mathrm{MHz}, \mathrm{CDCl}_{3}\right): 0.88(\mathrm{~d}, J=6.5 \mathrm{~Hz}, 3 \mathrm{H}) ; 0.97(\mathrm{~d}, J=6.5 \mathrm{~Hz}, 3 \mathrm{H}) ; 1.84-1.78(\mathrm{~m}$, $1 \mathrm{H}) ; 2.78-2.61(\mathrm{~m}, 6 \mathrm{H}) ; 3.41(\mathrm{~d}, J=3.3 \mathrm{~Hz}, 2 \mathrm{H}) ; 3.61-3.52(\mathrm{~m}, 4 \mathrm{H}) ; 3.82(\mathrm{dd}, J=3.2,11.6 \mathrm{~Hz}$, 1H), 4.11-4.07 (m, 1H); 7.30-7.25 (m, 5H). $\left.{ }^{13} \mathrm{C} \mathrm{NMR} \mathrm{(100.6} \mathrm{MHz,} \mathrm{CDCl}_{3}\right)$ : 20.3, 20.8, 27.4, $38.7,45.5,50.8,55.9,62.3,63.1,63.8,127.7,128.8,129.4,138.3$, 176.9. HRMS calcd for $\mathrm{C}_{17} \mathrm{H}_{27} \mathrm{~N}_{2} \mathrm{O}_{2}\left(\mathrm{M}^{+}+\mathrm{H}\right): 291.2073$; found, 291.2073 .

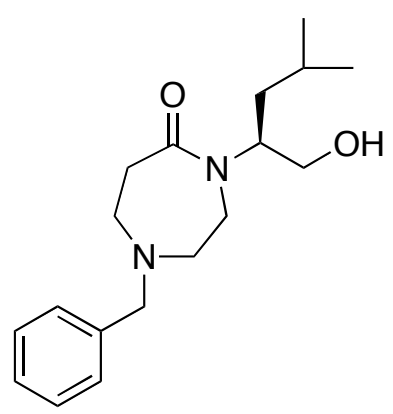

(S)-1-Benzyl-4-(1'-hydroxy-3'-methylpentan-2'-yl)-1,4-diazepan-5-one (5). Yellow oil; yield 87\%; $R_{f} 0.36$ (MeOH/EtOAc 1:9); [ $\left.\square\right]_{\mathrm{D}} \square 5.2$ (c 11.2, $\mathrm{CHCl}_{3}$ ). IR (neat): 3394, 1627 $\mathrm{cm}^{-1} .{ }^{1} \mathrm{H}$ NMR $\left(500 \mathrm{MHz}, \mathrm{CDCl}_{3}\right): 0.90(\mathrm{~d}, J=6.9 \mathrm{~Hz}, 3 \mathrm{H}) ; 0.92(\mathrm{~d}, J=3.6 \mathrm{~Hz}, 3 \mathrm{H}) ; 1.20-1.15$ (septet, $J=4.7 \mathrm{~Hz}, 1 \mathrm{H}) ; 1.34-1.29(\mathrm{~m}, 1 \mathrm{H}) ; 1.51(\mathrm{br} \mathrm{s}, 1 \mathrm{H}), 2.56(\mathrm{~s}, 2 \mathrm{H}) ; 2.63(\mathrm{~s}, 2 \mathrm{H}) ; 2.70(\mathrm{~s}$, $2 \mathrm{H}) ; 3.32(\mathrm{~s}, 2 \mathrm{H}) ; 3.60-3.35(\mathrm{~m}, 5 \mathrm{H}) ; 4.69(\mathrm{t}, J=4.1 \mathrm{~Hz}, 1 \mathrm{H}), 7.31-7.25(\mathrm{~m}, 5 \mathrm{H}) .{ }^{13} \mathrm{C}$ NMR (125.75 MHz, $\left.\mathrm{CDCl}_{3}\right): 22.1,22.8,25.0,37.2,38.5,43.4,50.6,54.1,55.9,62.7,63.6,127.1$, 128.3, 128.9, 138.0, 176.4. HRMS calcd for $\mathrm{C}_{18} \mathrm{H}_{29} \mathrm{~N}_{2} \mathrm{O}_{2}\left(\mathrm{M}^{+}+\mathrm{H}\right)$ : 305.2229; found, 305.2204 . 


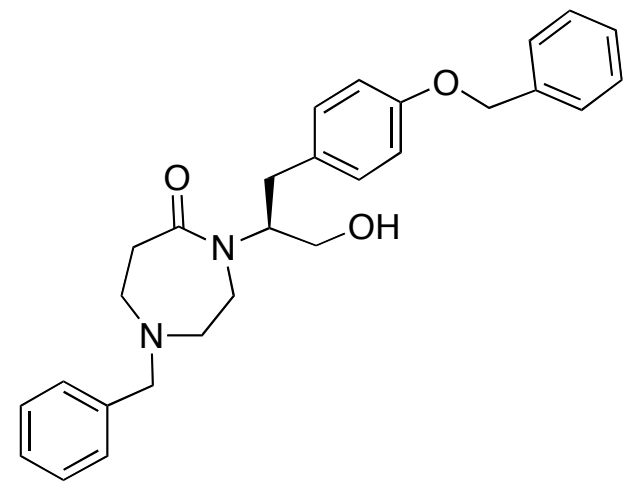

(S)-1-Benzyl-4-[3马(4G(benzyloxy)phenyl)-1'-hydroxypropan-2'-yl]-1,4-diazepan-5one (6). Yellow oil; yield 63\%; $R_{f} 0.39$ (MeOH/EtOAc 1:9); [ $\left.\square\right]_{\mathrm{D}} \square 39.3$ (c 2.9, $\mathrm{CHCl}_{3}$ ). IR (neat): 3373, $1627 \mathrm{~cm}^{-1} .{ }^{1} \mathrm{H}$ NMR (400 MHz, $\left.\mathrm{CDCl}_{3}\right): 2.40(\mathrm{~s}, 2 \mathrm{H}) ; 2.54(\mathrm{~d}, J=5.6 \mathrm{~Hz}, 1 \mathrm{H})$; $2.63(\mathrm{~s}, 2 \mathrm{H}) ; 2.83(\mathrm{~d}, J=6.5 \mathrm{~Hz}, 2 \mathrm{H}) ; 3.31(\mathrm{~d}, J=5.1 \mathrm{~Hz}, 2 \mathrm{H}) ; 3.75-3.45(\mathrm{~m}, 6 \mathrm{H}) ; 4.62(\mathrm{~d}, J=$ $4.7 \mathrm{~Hz}, 1 \mathrm{H}), 5.05$ (s, 2H), 6.93 (d, $J=1.4 \mathrm{~Hz}, 2 \mathrm{H}), 7.13$ (d, $J=8.4 \mathrm{~Hz}, 2 \mathrm{H}), 7.45-7.28(\mathrm{~m}, 10 \mathrm{H})$. ${ }^{13} \mathrm{C}$ NMR (100.6 MHz, $\left.\mathrm{CDCl}_{3}\right): 34.3,39.0,46.8,50.9,56.0,60.3,63.2,63.8,70.4,115.3,127.9$, $128.4,128.8,128.9 ; 129.5,129.7,130.4,138.3,157.9,176.8$. HRMS calcd for $\mathrm{C}_{28} \mathrm{H}_{33} \mathrm{~N}_{2} \mathrm{O}_{3}$ $\left(\mathrm{M}^{+}+\mathrm{H}\right)$ : 445.2491; found, 445.2469 .

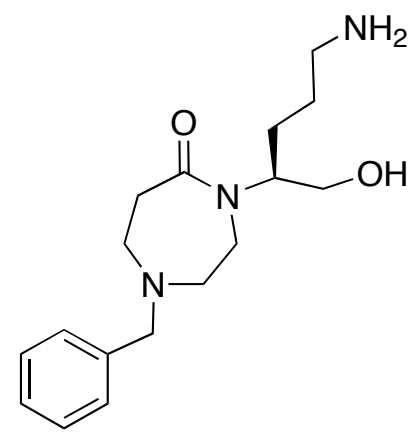

(S)-4-(5马Amino-1Ghydroxypentan-2Gyl)-1-benzyl-1,4-diazepan-5-one (7). Yellow oil; yield 84\%; $R_{f} 0.51$ (MeOH/EtOAc, 1:9); [0]D $\square 7.8$ (c 1.2, $\mathrm{CHCl}_{3}$ ). IR (neat): $3411,1623 \mathrm{~cm}^{-1}$. ${ }^{1} \mathrm{H}$ NMR $\left(500 \mathrm{MHz}, \mathrm{CDCl}_{3}\right): 1.71-1.68(\mathrm{~m}, 1 \mathrm{H}) ; 2.81-2.61(\mathrm{~m}, 2 \mathrm{H}) ; 3.90-3.41(\mathrm{~s}, 6 \mathrm{H}) ; 4.68(\mathrm{~s}$, $1 \mathrm{H})$; 7.35-7.07 (m, 5H). ${ }^{13} \mathrm{C}$ NMR (125.75 MHz, $\left.\mathrm{CDCl}_{3}\right)$ : 24.4, 28.2, 38.9, 44.1, 50.9, 52.9, 56.6, 57.0, 62.8, 63.0, 127.2, 128.7, 129.0, 138.2, 174.6. MS (EI) calcd for $\mathrm{C}_{17} \mathrm{H}_{27} \mathrm{~N}_{3} \mathrm{O}_{2}$ 305.2; found, $\mathrm{m} / \mathrm{e} 305.0$. 


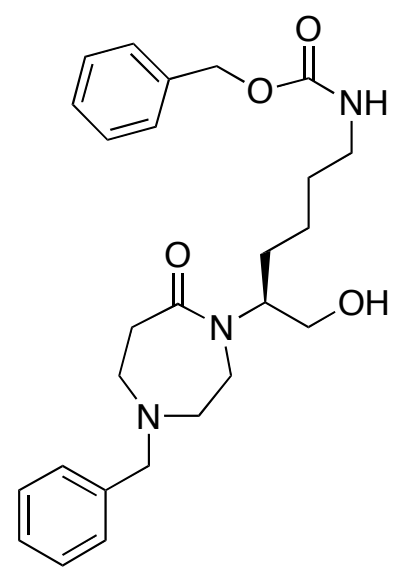

(S)-Benzyl-5-(4'-benzyl-7'-oxo-1',4'-diazepan-1'-yl)-6-hydroxyhexylcarbamate (8). Yellow oil; yield 88\%; $R_{f} 0.54$ (MeOH/EtOAc, 1:9); [ $\left.\square\right]_{\mathrm{D}} \square 6.9$ (c 0.97, $\mathrm{CHCl}_{3}$ ). IR (neat): 3329, 1716, $1628 \mathrm{~cm}^{-1} .{ }^{1} \mathrm{H}$ NMR (500 MHz, $\left.\mathrm{CDCl}_{3}\right): 1.68-1.33$ (m, 6H); 2.72-2.63 (m, 6H); 3.22-3.18 $(\mathrm{m}, 2 \mathrm{H}) ; 3.38(\mathrm{~s}, 1 \mathrm{H}) ; 3.95-3.49(\mathrm{~m}, 3 \mathrm{H}) ; 4.51(\mathrm{~s}, 1 \mathrm{H}) ; 4.93(\mathrm{~s}, 1 \mathrm{H}) ; 5.10(\mathrm{~s}, 2 \mathrm{H}) ; 7.37-7.11(\mathrm{~m}$, 10H). ${ }^{13} \mathrm{C}$ NMR (125.75 MHz, $\left.\mathrm{CDCl}_{3}\right): 23.1,27.6,29.4,38.3,40.5,44.1,50.4,55.8,56.4,62.7$, 63.6, 66.5, 127.2, 128.0, 128.3, 128.4, 128.9, 136.5, 137.8 (2C), 156.4, 176.3. HRMS calcd for $\mathrm{C}_{26} \mathrm{H}_{36} \mathrm{~N}_{3} \mathrm{O}_{4}\left(\mathrm{M}^{+}+\mathrm{H}\right)$ : 454.2706; found, 454.2723.

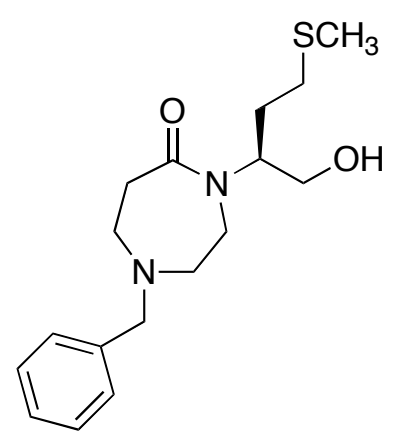

(S)-1-Benzyl-4-(1'-hydroxy-4'-(methylthio)butan-2'-yl)-1,4-diazepan-5-one (9). Yellow oil; yield 55\%; $R_{f} 0.28$ (EtOAc/MeOH, 9:1); [ $]_{\mathrm{D}} \square 2.0$ (c 1.1, $\mathrm{CHCl}_{3}$ ). IR (neat): 3411, $1623 \mathrm{~cm}^{-1} .{ }^{1} \mathrm{H}$ NMR (500 MHz, $\left.\mathrm{CDCl}_{3}\right): 1.83-1.27(\mathrm{~m}, 2 \mathrm{H}), 2.10(\mathrm{~s}, 3 \mathrm{H}) ; 2.78-2.47$ (m, 8H), $3.44(\mathrm{t}, J=4.6 \mathrm{~Hz}, 1 \mathrm{H}), 4.56-4.45(\mathrm{~m}, 6 \mathrm{H}) ; 4.56$ (s, 1H), 7.37-7.26 (m, 5H). ${ }^{13} \mathrm{C}$ NMR $(125.75$ $\left.\mathrm{MHz}, \mathrm{CDCl}_{3}\right)$ : 15.5, 27.9, 30.9, 41.2, 50.5, 51.2, 52.8, 61.9, 62.7, 63.2, 127.2, 128.3, 128.9, 137.7, 176.3. HRMS calcd for $\mathrm{C}_{17} \mathrm{H}_{27} \mathrm{~N}_{2} \mathrm{O}_{2} \mathrm{~S}\left(\mathrm{M}^{+}+\mathrm{H}\right)$ : 323.1793 ; found 323.1782. 


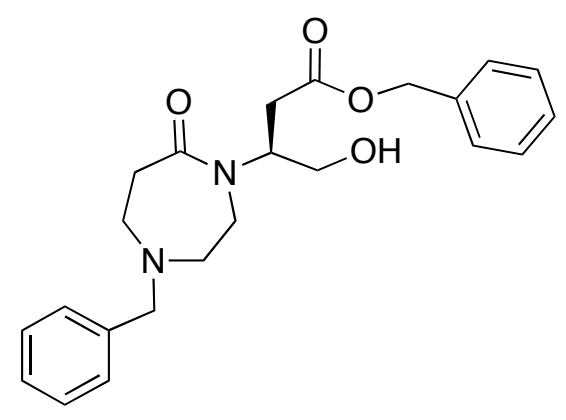

(S)-Benzyl-3-(4'-benzyl-7'-oxo-1',4'-diazepan-1'-yl)-6-hydroxybutanoate (10). Yellow

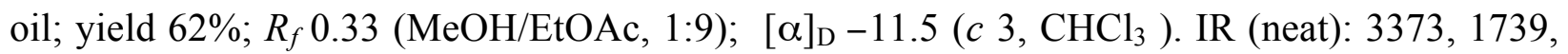
$1650 \mathrm{~cm}^{-1}$. ${ }^{1} \mathrm{H}$ NMR (400 MHz, $\left.\mathrm{CDCl}_{3}\right): 2.75-2.28(\mathrm{~m}, 6 \mathrm{H}) ; 3.59-3.44(\mathrm{~m}, 4 \mathrm{H}) ; 4.06-3.94(\mathrm{~m}$, $2 \mathrm{H}) ; 4.98(\mathrm{~d}, J=4.5 \mathrm{~Hz}, 1 \mathrm{H}) ; 5.18(\mathrm{dd}, J=1.4,12.8 \mathrm{~Hz}, 2 \mathrm{H}) ; 7.55-7.29(\mathrm{~m}, 10 \mathrm{H}) .{ }^{13} \mathrm{C}$ NMR $\left(100.6 \mathrm{MHz}, \mathrm{CDCl}_{3}\right)$ : 38.0, 48.7, 50.3, 55.6, 60.9, 61.4, 62.8, 67.2, 127.3, 128.4, 128.5, 128.6, 128.9, 129.1, 135.2, 137.9, 169.8, 175.7. FAB calcd for $\mathrm{C}_{23} \mathrm{H}_{28} \mathrm{~N}_{2} \mathrm{O}_{4}\left(\mathrm{M}^{+}-\mathrm{CH}_{2} \mathrm{Ph}+\mathrm{H}\right): 306.16$; found 307.1.

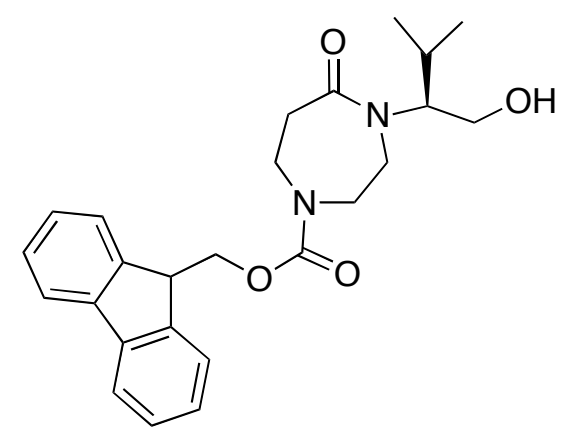

(S)-(9H-Fluoren-9 Gyl)methyl-4-(1Ghydroxy-3Gmethylbutan-2Gyl)-5-oxo-1,4-

diazepane-1-carboxylate (11). White foam; yield 63\%; $R_{f} 0.2(\mathrm{EtOAc} / \mathrm{MeOH}, 9: 1)$; $[\square]$ D $\square 1.7$ (c 1, $\mathrm{CHCl}_{3}$ ). IR (neat): 3456, 1695, $1638 \mathrm{~cm}^{-1} .{ }^{1} \mathrm{H} \mathrm{NMR}\left(400 \mathrm{MHz}, \mathrm{CDCl}_{3}\right): 0.87$ (d, $J=6.6 \mathrm{~Hz}$, $3 \mathrm{H}), 0.99(\mathrm{~d}, J=6.5 \mathrm{~Hz}, 3 \mathrm{H}), 1.84(\mathrm{~m}, 1 \mathrm{H}), 2.09$ (m, 1H), 2.52 (m, 2H), 3.58-3.34 (complex, $6 \mathrm{H}), 4.09(\mathrm{~m}, 1 \mathrm{H}), 4.23(\mathrm{~m}, 2 \mathrm{H}), 7.33(\mathrm{t}, J=7.4 \mathrm{~Hz}, 2 \mathrm{H}), 7.41(\mathrm{t}, J=7.4 \mathrm{~Hz}, 2 \mathrm{H}), 7.57(\mathrm{~d}, J=$ $7.4 \mathrm{~Hz}, 2 \mathrm{H}), 7.77$ (d, $J=7.5 \mathrm{~Hz}, 2 \mathrm{H}) .{ }^{13} \mathrm{C} \mathrm{NMR}\left(100.6 \mathrm{MHz}, \mathrm{CDCl}_{3}\right): 20.3,20.7,27.3,39.8$, $42.4,46.2$, 47.5, 47.9, 62.1, 63.6, 67.3, 67.6, 120.4, 125.2, 127.5, 128.2, 141.8, 144.3, 155.8, 175.9. HRMS calcd for $\mathrm{C}_{25} \mathrm{H}_{31} \mathrm{~N}_{2} \mathrm{O}_{4}\left(\mathrm{M}+\mathrm{H}^{+}\right)$: 423.2275, found 423.2284. 


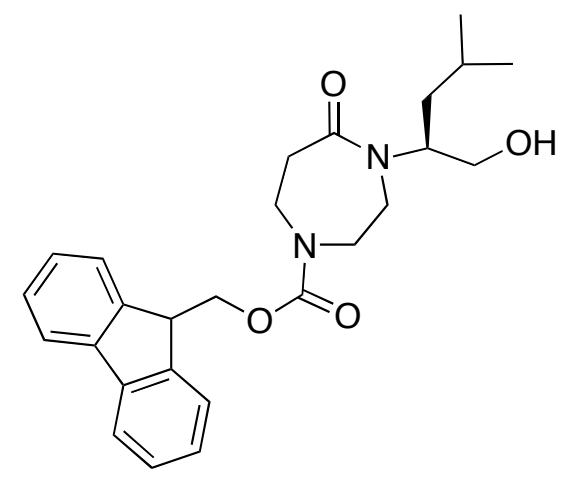

\section{(S)-(9H-Fluoren-9Gyl)methyl-4-(1Ghydroxy-3马methylpentan-2 Gyl)-5-oxo-1,4-}

diazepane-1-carboxylate (12). Colorless oil, yield 68\%; $R_{f} 0.22(\mathrm{EtOAc} / \mathrm{MeOH}, 9: 1)$; [ $]_{\mathrm{D}}$ $\square 0.8$ (c 1.05, $\left.\mathrm{CHCl}_{3}\right)$. IR (neat): $3441,1685,1629 \mathrm{~cm}^{-1} .{ }^{1} \mathrm{H}$ NMR (400 MHz, $\left.\mathrm{CDCl}_{3}\right): 0.92$ (d, $J$ $=6.4,6 \mathrm{H}), 1.18(\mathrm{~m}, 1 \mathrm{H}), 1.32(\mathrm{~m}, 1 \mathrm{H}), 2.46(\mathrm{~m}, 1 \mathrm{H}), 2.51(\mathrm{~m}, 3 \mathrm{H}), 3.74-2.97$ (complex, 8H), $4.23(\mathrm{t}, J=5.6 \mathrm{~Hz}, 1 \mathrm{H}), 4.57$ (quartet, $J=7.4,5.9 .2 \mathrm{H}), 4.72(\mathrm{~m}, 1 \mathrm{H}), 7.32(\mathrm{t}, J=7.4,2 \mathrm{H}), 7.40$ $(\mathrm{t}, J=7.3,2 \mathrm{H}), 7.56(\mathrm{~d}, J=7.2,2 \mathrm{H}), 7.76(\mathrm{~d}, J=7.5,2 \mathrm{H}) .{ }^{13} \mathrm{C} \mathrm{NMR}\left(100.6 \mathrm{MHz}, \mathrm{CDCl}_{3}\right): 22.6$, 23.7, 25.5, 37.6, 39.9, 42.5, 44.7, 47.9, 54.8, 64.3, 67.6, 120.4, 125.2, 127.5, 128.2, 141.8, 144.2. HRMS calcd for $\mathrm{C}_{26} \mathrm{H}_{32} \mathrm{~N}_{2} \mathrm{O}_{4}\left(\mathrm{M}^{+}+\mathrm{H}^{+}\right): 437.2418$, found 437.2418.

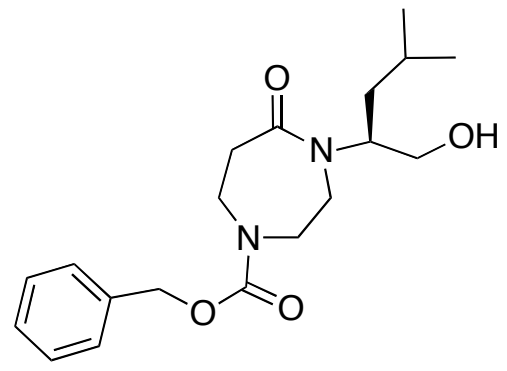

\section{(S)-Benzyl-4-(1Ghydroxy-4Gmethylpentan-2马yl)-5-oxo-1,4-diazepan-1-carboxylate}

(13). Colorless oil, yield 52\%; $R_{f} 0.31$ (EtOAc/MeOH. 9:1); [ $]_{\mathrm{D}} \square 1.1$ (c 1.03, $\mathrm{CHCl}_{3}$ ). IR (neat): 3436, 1695, $1649 \mathrm{~cm}^{-1} .{ }^{1} \mathrm{H}$ NMR $\left(400 \mathrm{MHz}, \mathrm{CDCl}_{3}\right): 0.90(\mathrm{~d}, J=3.9,3 \mathrm{H}), 0.91(\mathrm{~d}, J=$ 3.7, 3H), $1.17(\mathrm{~m}, 2 \mathrm{H}), 1.33(\mathrm{~m}, 1 \mathrm{H}), 1.47(\mathrm{~m}, 1 \mathrm{H}), 2.68(\mathrm{~m} \mathrm{2H}), 3.63-3.35$ (complex, 7H), 3.74 (dd, $J=14.7,6.6,2 \mathrm{H}), 4.74(\mathrm{~m}, 1 \mathrm{H}), 5.13(\mathrm{~s}, 2 \mathrm{H}), 7.34(\mathrm{~m}, 5 \mathrm{H}) .{ }^{13} \mathrm{C} \mathrm{NMR}\left(100.6 \mathrm{MHz}, \mathrm{CDCl}_{3}\right)$ : 22.6, 23.7, 25.5, 37.7, 40.0, 42.3, 44.5, 47.8, 54.6, 63.8, 68.0, 128.4, 128.9, 129.4, 136.7, 155.8, 176.1 HRMS calcd for $\mathrm{C}_{22} \mathrm{H}_{27} \mathrm{~N}_{2} \mathrm{O}_{4}\left(\mathrm{M}^{+}+\mathrm{H}\right): 349.2127$, found 349.2121. 


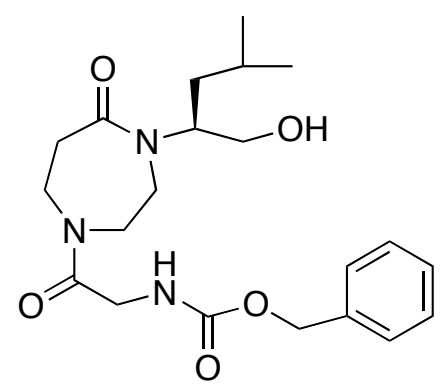

(S)-Benzyl-2-[(4'-(1'-hydroxy-4'-methylpentan-2'-yl)-5-oxo-1,4-diazepan-1-yl]-2-

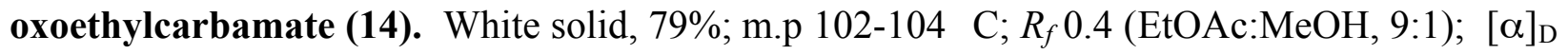
$\square 23.1$ (c 3.1, $\mathrm{CHCl}_{3}$ ). IR (neat): 3406, 1718, $1637 \mathrm{~cm}^{-1} .{ }^{1} \mathrm{H}$ NMR (500 MHz, $\mathrm{CDCl}_{3}$ ): 0.93 (d, $J$ $=3.5 \mathrm{~Hz}, 3 \mathrm{H}) ; 0.94(\mathrm{~d}, J=3.1 \mathrm{~Hz}, 3 \mathrm{H}) ; 1.26-1.21$ (septet, $J=5 \mathrm{~Hz}, 1 \mathrm{H}) ; 1.51-1.37$ (m, 2H); 1.70 (br s, 2H); 2.09 (br s, 1H); 2.78-2.73 (m, 2H); 3.59-3.37 (m, 6H); 4.12-4.06 (m, 2H), 4.78-4.75 (q, $J=4.5 \mathrm{~Hz}, 1 \mathrm{H}), 5.14(\mathrm{~s}, 2 \mathrm{H}) ; 5.83(\mathrm{~s}, 1 \mathrm{H}) ; 7.39-7.28(\mathrm{~m}, 5 \mathrm{H}) .{ }^{13} \mathrm{C}$ NMR $(125.75 \mathrm{MHz}$, $\mathrm{CDCl}_{3}$ ): 22.1, 23.2, 25.1, 37.1, 39.2, 42.6; 43.8, 45.3, 47.7, 54.3, 63.8, 66.9, 127.9, 128.3, 128.4, 136.2, 156.2, 167.1, 175.1. HRMS calcd for $\mathrm{C}_{21} \mathrm{H}_{32} \mathrm{~N}_{3} \mathrm{O}_{5}\left(\mathrm{M}^{+}+\mathrm{H}\right): 406.2342$; found, 406.2344 .

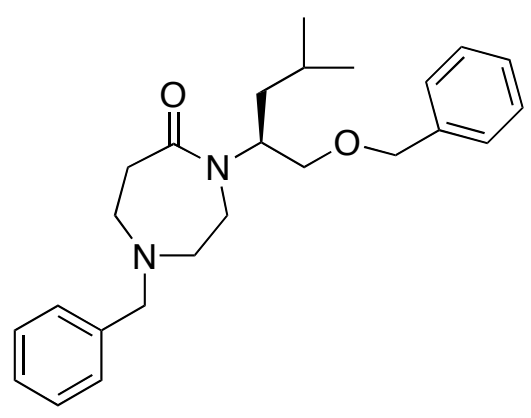

(S)-1-Benzyl-4-[1'-(benzyloxy)-4'-methylpentan-2'-yl]-1,4-diazepan-5-one. Sodium hydride (60\% in mineral oil, $0.272 \mathrm{~g}, 11.3 \mathrm{mmol})$ was suspended in DMF $(5 \mathrm{~mL})$ and hexane (1 $\mathrm{mL})$, cooled to $-78{ }^{\circ} \mathrm{C}$, and $5(1.15 \mathrm{~g}, 3.7 \mathrm{mmol})$ in DMF was added dropwise. The resulting mixture was stirred for $15 \mathrm{~min}$, at which times time benzyl bromide $(0.71 \mathrm{~g}, 0.23 \mathrm{~mL}, 4.2 \mathrm{mmol})$ was added dropwise and the reaction mixture was allowed to stir at room temperature for $3 \mathrm{~h}$. The reaction mixture was diluted with ethyl acetate $(50 \mathrm{~mL})$, washed with ammonium chloride solution, and dried over anhydrous $\mathrm{Na}_{2} \mathrm{SO}_{4}$. The crude mixture was purified through column chromatography to afford the title compound. Yellow oil; yield 81\%; $\mathrm{R}_{\mathrm{f}} 0.78$ (EtOAc/hexane 
1:1); $[\square]_{\mathrm{D}} \square 11.4$ (c 11, $\mathrm{CHCl}_{3}$ ). IR (neat): $1640 \mathrm{~cm}^{-1} .{ }^{1} \mathrm{H}$ NMR (500 MHz, $\left.\mathrm{CDCl}_{3}\right): 0.92$ (d, $J=$ $6.6 \mathrm{~Hz}, 3 \mathrm{H}) ; 0.95(\mathrm{~d}, J=6.4 \mathrm{~Hz}, 3 \mathrm{H}) ; 1.23-1.19(\mathrm{~m}, 1 \mathrm{H}) ; 1.51-1.42(\mathrm{~m}, 2 \mathrm{H}) ; 2.71-2.45(\mathrm{~m}, 6 \mathrm{H})$; $3.52-3.32(\mathrm{~m}, 6 \mathrm{H}), 4.40(\mathrm{~d}, J=11.1 \mathrm{~Hz}, 1 \mathrm{H}) ; 4.50(\mathrm{~d}, J=11.3 \mathrm{~Hz}, 1 \mathrm{H}) ; 4.92(\mathrm{~s}, 1 \mathrm{H}) ; 7.33-7.13$ $(\mathrm{m}, 10 \mathrm{H}) .{ }^{13} \mathrm{C} \mathrm{NMR}\left(125.75 \mathrm{MHz}, \mathrm{CDCl}_{3}\right): 22.2,23.4,25.0,37.7,38.8,43.7,50.6,51.0,56.3$, $62.8,71.5,72.7,127.1,127.5,127.6,128.3,128.6,128.9,138.0,138.2$, 175.1. HRMS calcd for $\mathrm{C}_{25} \mathrm{H}_{35} \mathrm{~N}_{2} \mathrm{O}_{2}\left(\mathrm{M}^{+}+\mathrm{H}\right):$ 395.2699; found, 395.2679.

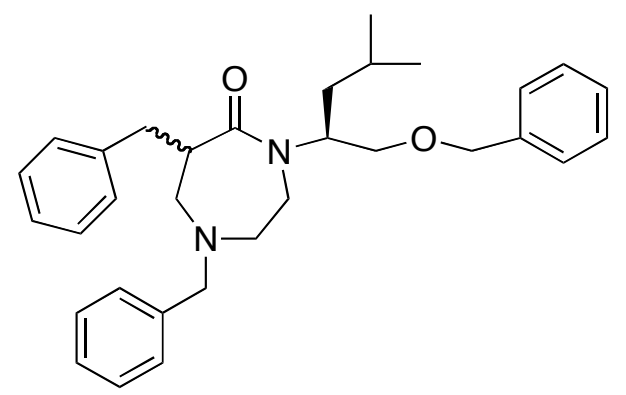

(S)-1,6-Dibenzyl-4-[1'-(benzyloxy)-4'-methylpentan-2'-yl]-1,4-diazepan-5-one. To a solution of the above $O$-benzyl 1,4-diazepan-5-one (0.540 g, $1.36 \mathrm{mmol})$ in THF/HMPA mixture $(5 \mathrm{~mL} / 1 \mathrm{~mL})$ at $-78{ }^{\circ} \mathrm{C}$ was added LiHMDS (1.15 g, $\left.6.84 \mathrm{mmol}\right)$. After being allowed to stir for $15 \mathrm{~min}$, benzyl bromide $(0.325 \mathrm{~mL}, 2.73 \mathrm{mmol})$ was added dropwise. The reaction mixture was allowed to stir at $-78{ }^{\circ} \mathrm{C}$ for $1 \mathrm{~h}$, when it was allowed to warm to room temperature and allowed to stir for $2 \mathrm{~h}$. The reaction mixture was diluted with saturated aqueous $\mathrm{NH}_{4} \mathrm{Cl}$ and extracted with ethyl acetate $(50 \mathrm{~mL})$. The organic layer was washed with brine and water and dried over anhydrous $\mathrm{Na}_{2} \mathrm{SO}_{4}$. Column chromatography afforded the desired compound as a mixture of diastereomers (2:1). Yellow oil; yield 56\%; $R_{f} 0.70$ (EtOAc, 100\%); IR (neat): $1646 \mathrm{~cm}^{-1} .{ }^{1} \mathrm{H}$ NMR (500 MHz, $\left.\mathrm{CDCl}_{3}\right)$ : 1.08-0.85 (m, 6H); $1.31(\mathrm{~m}, 1 \mathrm{H}) ; 1.32-1.31(\mathrm{~m}, 2 \mathrm{H}) ; 2.09$ (br s, 2H); 2.84 (br s, $1 \mathrm{H}) ; 2.86(\mathrm{~d}, J=9.5 \mathrm{~Hz}, 2 \mathrm{H}) ; 3.16(\mathrm{~m}, 1 \mathrm{H}) ; 3.56-3.36(\mathrm{~m}, 8 \mathrm{H}) ; 4.60-4.48(\mathrm{~m}, 2 \mathrm{H})$; 4.60 (br s, 1H) 7.93-7.22 (m, 15H). ${ }^{13} \mathrm{C} \mathrm{NMR}\left(125.75 \mathrm{MHz}, \mathrm{CDCl}_{3}\right): 22.1,23.2,25.0,36.0,37.5$, $38.3,42.7,46.1,50.6,55.2,56.0,56.4,62.6,71.1,71.9,72.8,125.8,125.8,126.9,127.5,127.6$, $127.7,128.2,128.2,129.0,138.2,140.3,175.9,176.3$. HRMS calcd for $\mathrm{C}_{32} \mathrm{H}_{41} \mathrm{~N}_{2} \mathrm{O}_{2}\left(\mathrm{M}^{+}+\mathrm{H}\right)$ : 485.3168; found, 485.3141 . 


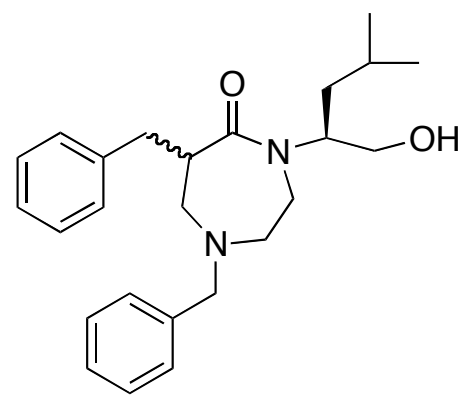

(S)-1,6-Dibenzyl-4-(1'-hydroxy-4'-methylpentan-2'-yl)-1,4-diazepan-5-one (15). Mixture of diastereomers (2:1), yellow oil; yield 63\%; $R_{f} 0.27$ (EtOAc, 100\%); IR (neat): 3324 , $1639 \mathrm{~cm}^{-1} .{ }^{1} \mathrm{H}$ NMR (500 MHz, $\left.\mathrm{CDCl}_{3}\right)$ : 0.95-0.87 (m, 6H); 1.01-1.00 (m, 1H); 1.43 (m, 2H); $1.84(\mathrm{~s}, 1 \mathrm{H}) ; 2.68 .2 .66(\mathrm{~m}, 1 \mathrm{H}) ; 3.01-2.89(\mathrm{~m}, 1 \mathrm{H}) ; 3.46-3.29(\mathrm{~m}, 6 \mathrm{H}) ; 4.60-4.44(\mathrm{~m}, 2 \mathrm{H}) ; 5.08$ (br s, 1H). ${ }^{13} \mathrm{C}$ NMR (125.75 MHz, $\mathrm{CDCl}_{3}$ ): 21.9, 22.5, 23.1, 25.0, 35.7, 37.5, 38.3, 47.6, 48.5, $70.9,72.7,72.8,125.8,127.5,128.3,129.1,137.9,138.1,140.3,175.6$. HRMS calcd for $\mathrm{C}_{25} \mathrm{H}_{35} \mathrm{~N}_{2} \mathrm{O}_{2}\left(\mathrm{M}^{+}+\mathrm{H}\right):$ 395.2699; found, 395.2688.

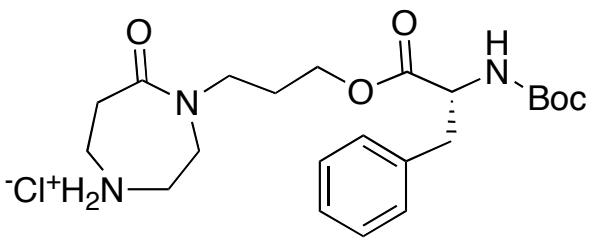

\section{Solid Phase Synthesis of (S)-3-[7-Oxo-1,4-diazepan-1-yl]propyl-2-(tert-} butoxycarbonyl)-3-phenylpropanoate hydro chloride (16). A mixture of Merrifield resin (2 g, $3 \mathrm{mmol})$, piperidone hydrochloride $(1.4 \mathrm{~g}, 9 \mathrm{mmol})$ and diisopropylethylamine $(2.6 \mathrm{~mL}, 15$ $\mathrm{mmol})$ in DMF $(15 \mathrm{~mL})$ was shaken at $50{ }^{\circ} \mathrm{C}$ for $12 \mathrm{~h}$. The resin was filtered and then resubjected to the above procedure to ensure complete loading. The resin was then washed with DMF (100 $\mathrm{mL}), \mathrm{CH}_{2} \mathrm{Cl}_{2}(100 \mathrm{~mL})$, and ether $(100 \mathrm{~mL})$ and dried under vacuum for several hours to a constant weight of $2.42 \mathrm{~g}$. To a suspension of above resin and 1-azidopropan-3-ol (1.7 g, 9 mmol) in $\mathrm{CH}_{2} \mathrm{Cl}_{2}$ was added $\mathrm{BF}_{3} \bullet \mathrm{Et}_{2} \mathrm{O}(1.9 \mathrm{~mL}, 15 \mathrm{mmol})$ at room temperature. After shaking for 1 day, it was filtered and treated with $15 \% \mathrm{KOH}(10 \mathrm{~mL})$ in THF $(10 \mathrm{~mL})$ for $2 \mathrm{~h}$. The resin was filtered, washed with water $(200 \mathrm{~mL})$, THF $(100 \mathrm{~mL}), \mathrm{CH}_{2} \mathrm{Cl}_{2}(100 \mathrm{~mL})$, and ether (100 $\mathrm{mL})$, and then dried. A mixture of the resulting resin (1.1 g, $1.2 \mathrm{mmol}, 1$ equiv) and BocN-Phe$\mathrm{OH}$ (0.95 g, $3.6 \mathrm{mmol}$ ), and DMAP (0.04 g, $0.3 \mathrm{mmol}, 0.1$ equiv) in $\mathrm{CH}_{2} \mathrm{Cl}_{2}$ (10mL) was shaken together at room temperature for a day. The resin was filtered, washed with water $(100 \mathrm{~mL})$, aqueous $\mathrm{NaHCO}_{3}(100 \mathrm{~mL}), \mathrm{MeOH}(100 \mathrm{~mL})$, THF $(100 \mathrm{~mL}), \mathrm{CH}_{2} \mathrm{Cl}_{2}(100 \mathrm{~mL})$ and ether (100 $\mathrm{mL})$, and dried to constant weight. The resin was suspended in $\mathrm{CH}_{2} \mathrm{Cl}_{2}(10 \mathrm{~mL})$ and allowed to 
stand for $5 \mathrm{~min}$. Chloroethylchloroformate $(0.4 \mathrm{~mL}, 3.7 \mathrm{mmol})$ was introduced and the suspension allowed to stir for $12 \mathrm{~h}$, after which time the resin was filtered off and washed with $\mathrm{CH}_{2} \mathrm{Cl}_{2}$. The residue obtained after the concentration of filtrate was subjected to flash chromatography with EtOAc to furnish $0.21 \mathrm{~g}$ of the chloroformate derivative. This was allowed to reflux in $5 \mathrm{~mL}$ of methanol for $1 \mathrm{~h}$, after which evaporation of the solvent furnished $0.19 \mathrm{~g}$ of title compound (33\% yield from piperidinone). White foam; yield 33\%; IR (neat): 3406, 1718, $1637 \mathrm{~cm}^{-1}$. ${ }^{1} \mathrm{H}$ NMR (400 MHz, $\left.\mathrm{CDCl}_{3}\right): 1.17-1.11(\mathrm{~m}, 2 \mathrm{H}) ; 1.41(\mathrm{~s}, 9 \mathrm{H}) ; 1.93-1.59(\mathrm{~m}, 6 \mathrm{H})$; $3.06(\mathrm{~s}, 4 \mathrm{H}) ; 3.78-3.37(\mathrm{~m}, 6 \mathrm{H}) ; 4.07$ (br s, 1H); $4.52-4.44(\mathrm{~m}, 2 \mathrm{H}), 5.08(\mathrm{~d}, J=7.3 \mathrm{~Hz}, 1 \mathrm{H})$; 7.31-7.17 (m, 5H). ${ }^{13} \mathrm{C}$ NMR (100.6 MHz, $\left.\mathrm{CDCl}_{3}\right): 25.3,25.9,27.5,28.7,34.3$, 34.6; 38.7; 46.2; 49.6, 55.0, 62.8, 80.4, 127.4, 129.0, 129.6, 136.4, 155.6, 172.3. HRMS calcd for $\mathrm{C}_{22} \mathrm{H}_{34} \mathrm{~N}_{3} \mathrm{O}_{5}$ $\left(\mathrm{M}^{+}+\mathrm{H}\right):$ 420.2498; found, 420.2487 .

\section{References}

1. (a) Giannis, A; Sandhoff, K. Angew Chem., Int. Ed. Engl. 1989, 28, 218-220. (b) Qualgliato, D.A.; Andrae, P.M.; Matelan, E.M. J. Org. Chem. 2000, 65, 5037-5042. (c) Meyers, A.I.; McKennon, M.J. J. Org. Chem. 1993, 58, 3568-3571.

2. (a). Zaloom, J.; Roberts, D.C. J. Org. Chem. 1981, 46, 5173-5176 (b). Vasella, A.; Witzig, C.; Chiara, J.L.; Lomas, M. M. Helv. Chim. Acta. 1991, 74, 2073-2077.

3. Cooper, C.S.; Klock, P.L.; Chu, D.T.W.; Hardy, D.J.; Swanson, R. N.; Plattner, J.J. J. Med. Chem. 1992, 35, 1392-1398. 

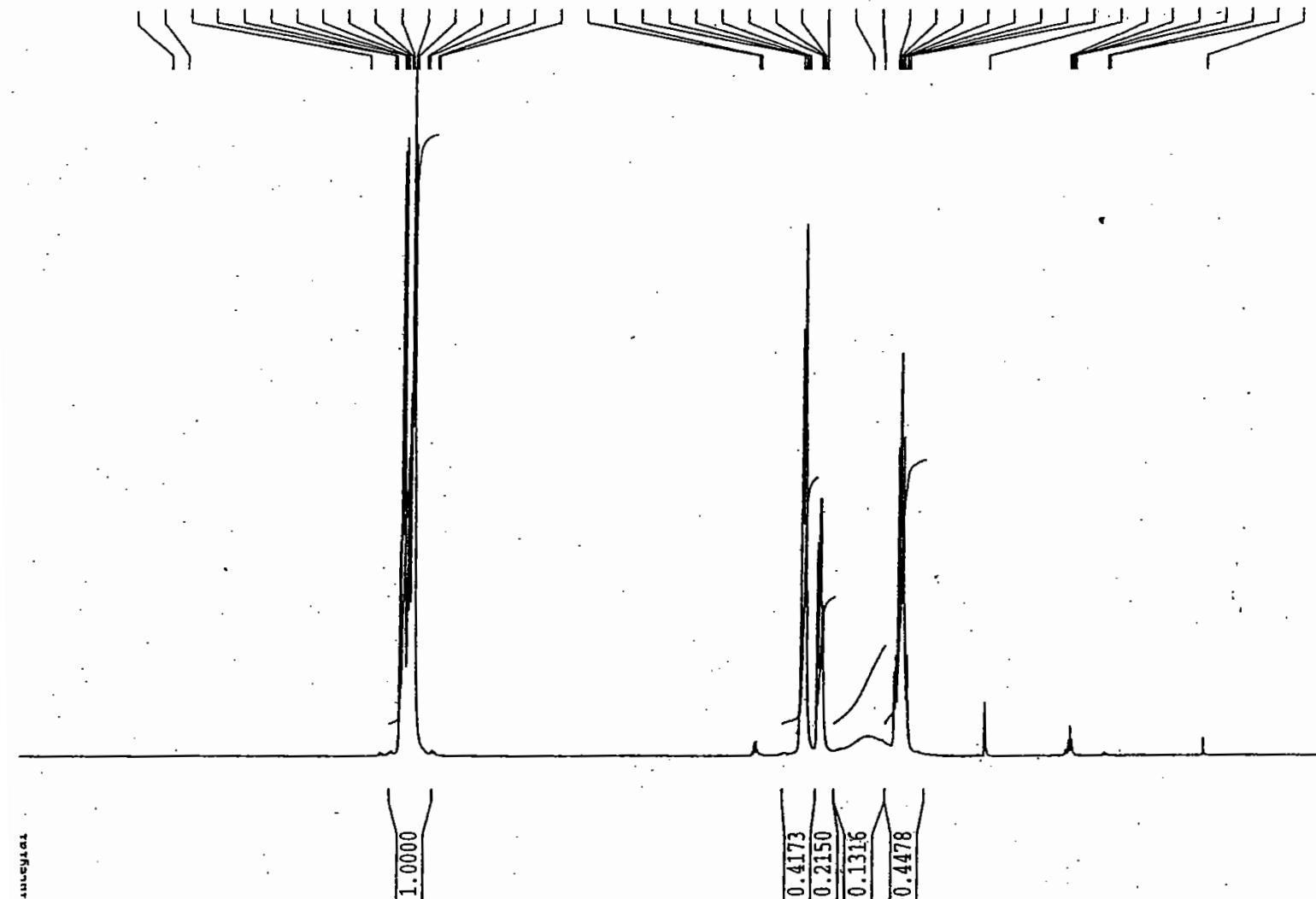<smiles>NC(CO)Cc1ccccc1</smiles>
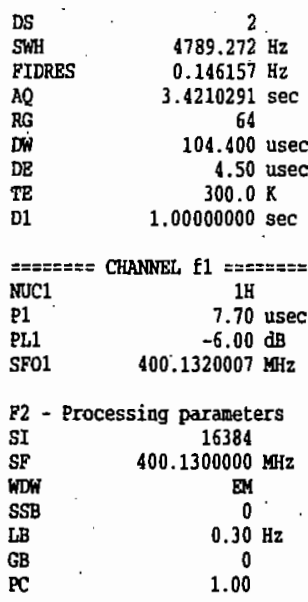

1D MIR plot parameters

$\begin{array}{lc}C X & 20.00 \mathrm{~cm} \\ \text { CY } & 12.50 \mathrm{~cm} \\ \text { F1P } & 11.000 \mathrm{ppm} \\ \text { F1 } & 4401.43 \mathrm{~Hz} \\ \text { F2P } & -1.000 \mathrm{ppm} \\ \text { F2 } & -400.13 \mathrm{~Hz} \\ \text { PFHCH } & 0.60000 \mathrm{ppm} / \mathrm{cm} \\ \text { HZCH } & 240.07800 \mathrm{~Hz} / \mathrm{cm}\end{array}$

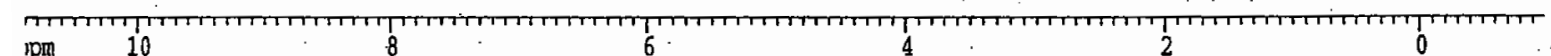
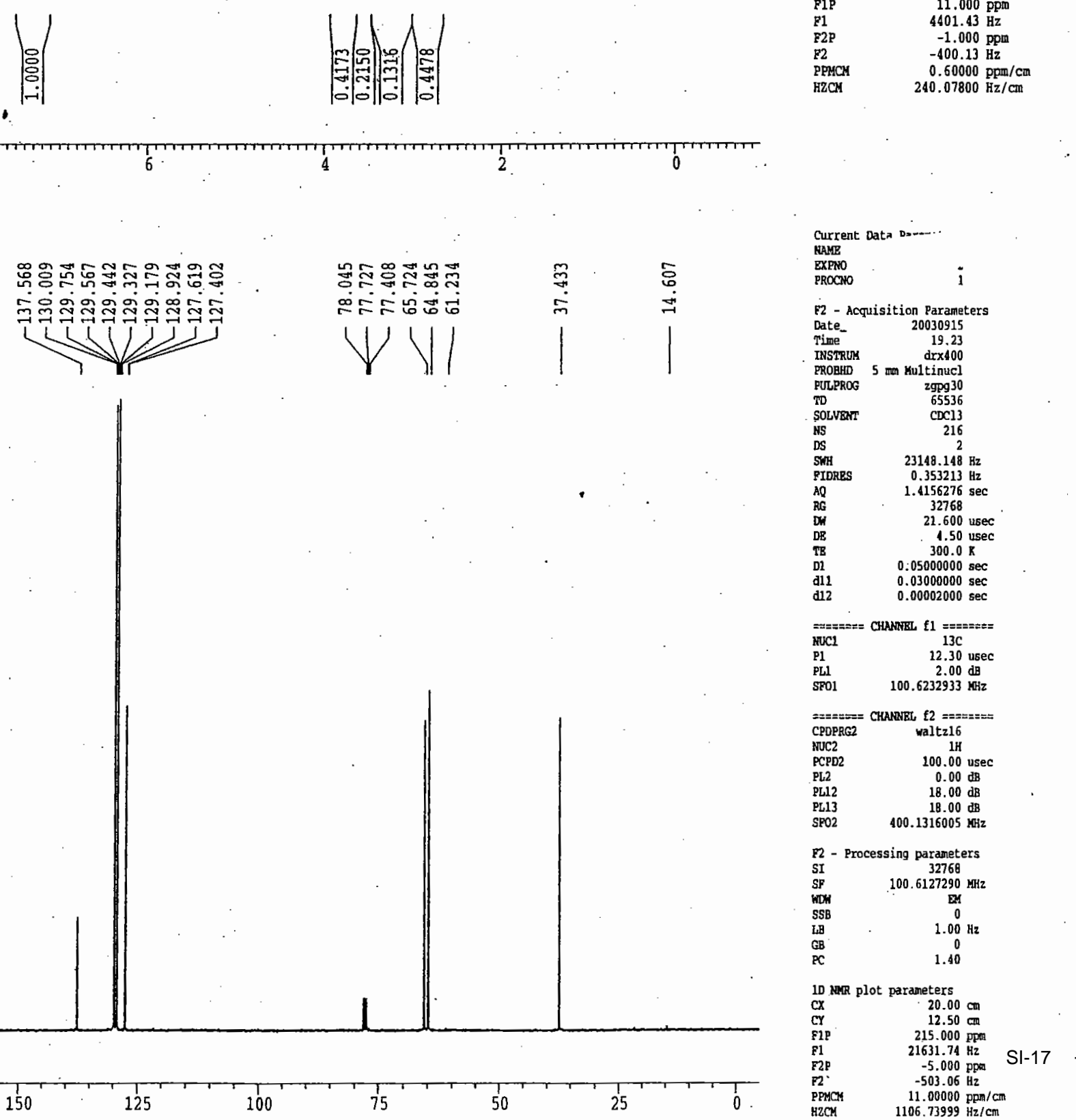

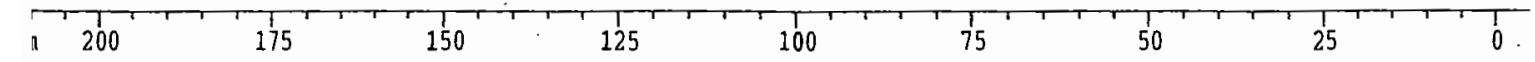



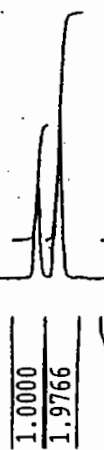

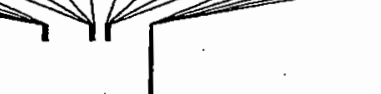

$\mathrm{N}_{3}$

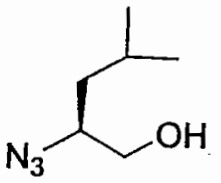

$1 \mathrm{~b}$

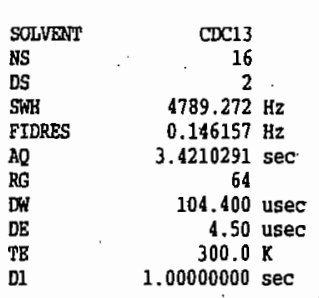

$==:= \pm==$ CHANEL EI =:s=s=s=

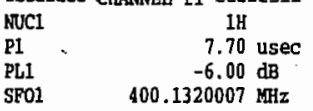

F2 - Processing paraneters

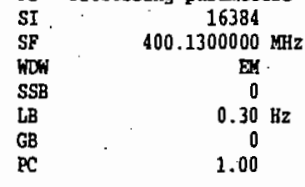

10 MR plot parameters

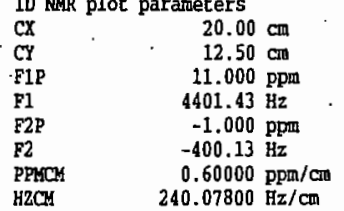

Girrant n....

sxim

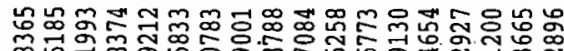

₹EЕ

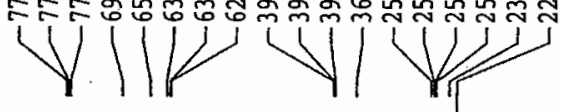

P2 - Acquisition Paraneters

Date_. 20030619

$\begin{array}{lr}\text { Time } & 19.13 \\ \text { INSTRUL } & \text { drx400 }\end{array}$ PROBHD 5 Multinucl $\begin{array}{lr}\text { POLPFOG } & \text { zgpg30 } \\ \text { TD } & 65536\end{array}$

Torn

SOLV

DS

SWF 23148.148

$23148.148 \mathrm{~Hz}$
FIDRRS $\quad 0.353213 \mathrm{~Hz}$

AQ $\quad 1.4156276 \mathrm{sec}$ 32768 21.600 usec 4.50 usec $300.0 \mathrm{~K}$ $0.05000000 \mathrm{sec}$

$0.03000000 \mathrm{sec}$ $0.00002000 \mathrm{sec}$

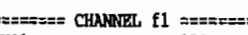

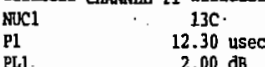
$\begin{array}{lr}\text { PL1. } & 2.00 \mathrm{~dB} \\ \text { SFO1 } & 100.6232933 \mathrm{dHz}\end{array}$ $==== \pm=3=$ CHANNRL $f 2$ =:==:=:= CPDPRG2 waltz16 NuC2 - $1 \mathrm{H}$ PCPD2 $\quad 100.00$ use PL2 $0.00 \mathrm{~dB}$ PL13 13 SFO2 400.1316005

22 - Processing paraneters $\begin{array}{lc}\text { SI } & 32768 \\ \text { SF } & 100.6127290 \text { tWH }\end{array}$ WDW 100.6127290 LB $\quad 1.00 \mathrm{~Hz}$ $\mathrm{GB}$

1D NTR plot parameters $\mathrm{CX} \quad 20.00 \mathrm{~cm}$ $\begin{array}{lr}\text { CY } & 12.50 \mathrm{~cm} \\ \text { FIP } & 215.000\end{array}$ F1 $\quad 21631.74 \mathrm{~Hz}$ $\mathrm{F2P} \quad-5.000 \mathrm{pp}$ 

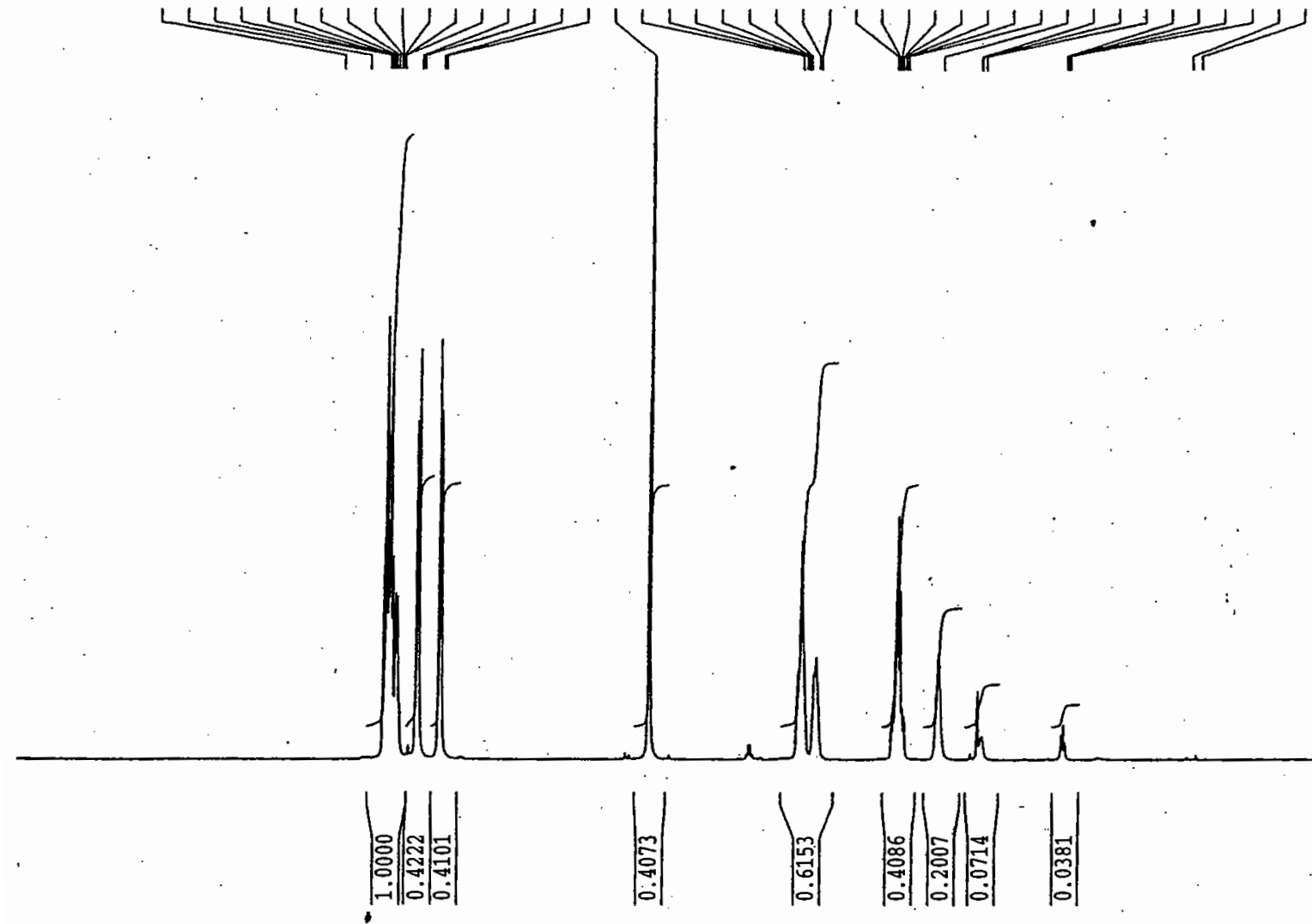

III. 10
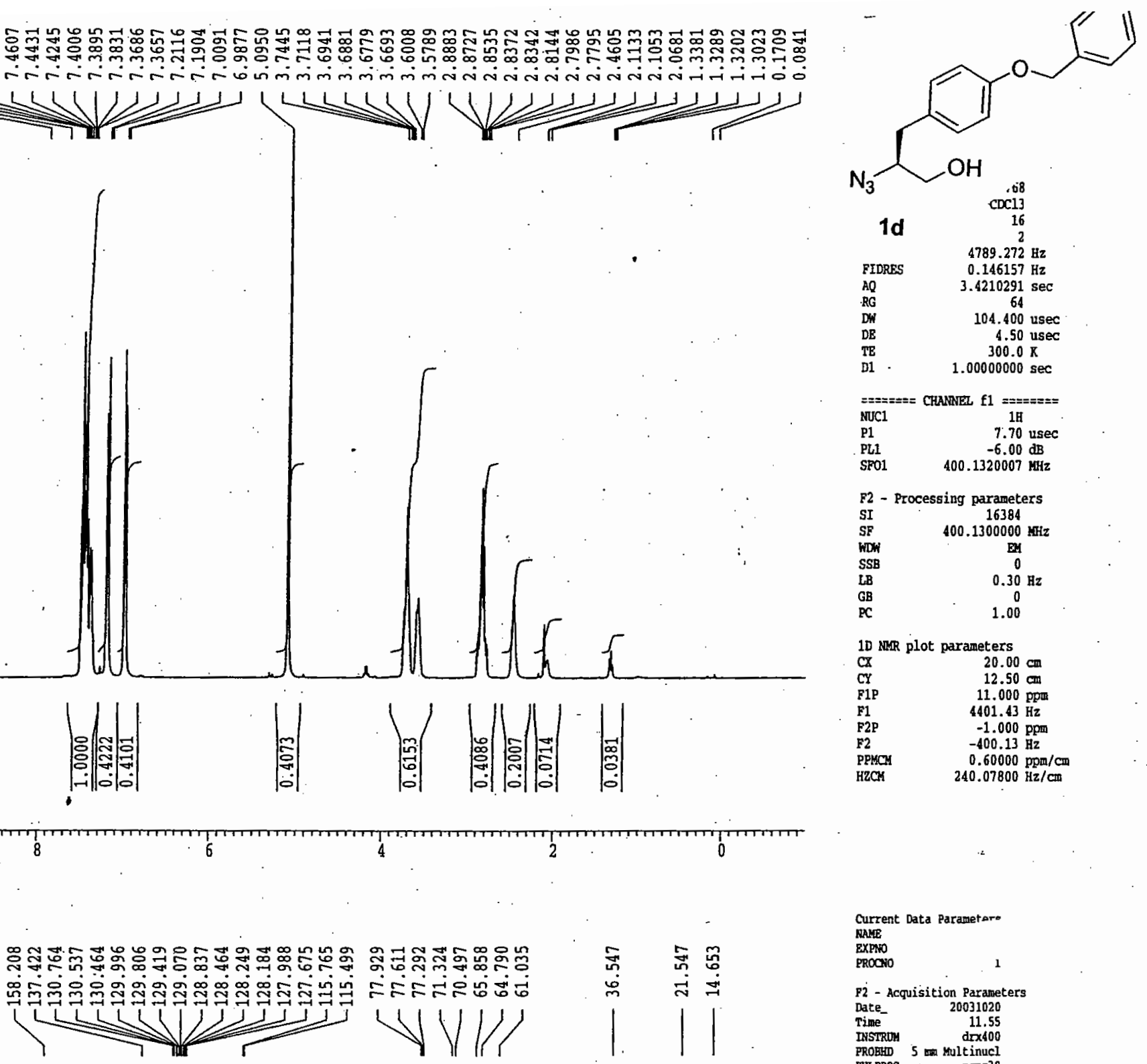

F2 - Processing parameters

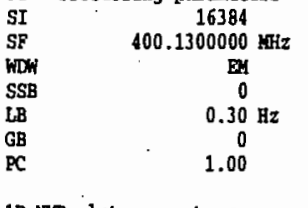

1D NaR plot parameters

$\begin{array}{lc}\text { 1D NaR plot paraneters } \\ \text { CX } & 20.00 \mathrm{~cm} \\ \text { CY } & 12.50 \mathrm{~cm} \\ \text { F1P } & 11.000 \mathrm{ppm} \\ \text { F1 } & 4401.43 \mathrm{~Hz} \\ \text { F2P } & -1.000 \mathrm{ppm} \\ \text { F2 } & -400.13 \mathrm{~Hz} \\ \text { PPHOM } & 0.60000 \mathrm{ppm} / \mathrm{cm} \\ \text { HZCM } & 240.07800 \mathrm{~Hz} / \mathrm{cm}\end{array}$

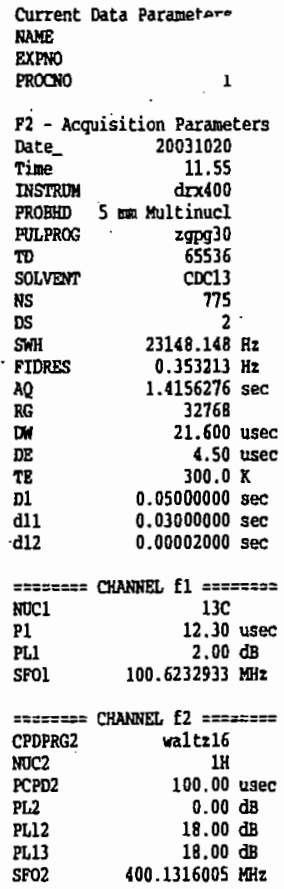

F2 - Processing paraneters

F2 - Processing paramete 32768

SF $\quad 100.6127290 \mathrm{MH}$

$\begin{array}{lc}\text { SSB } & 0 \\ \text { LB } & 1.00 \mathrm{~Hz}\end{array}$

$\mathrm{GB} \quad 1.40$

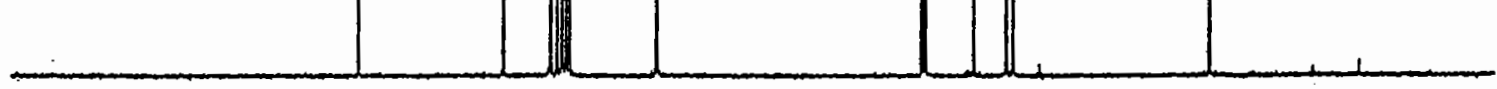

10 NAR plot parameters

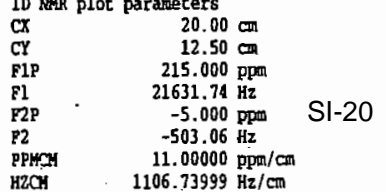




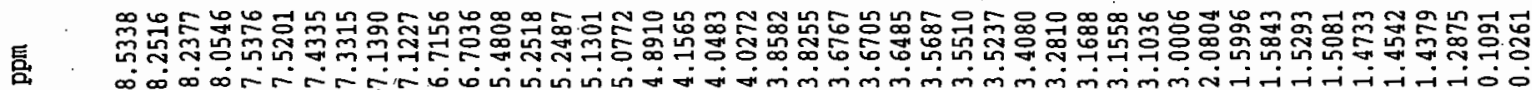

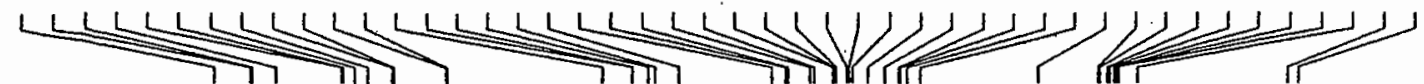

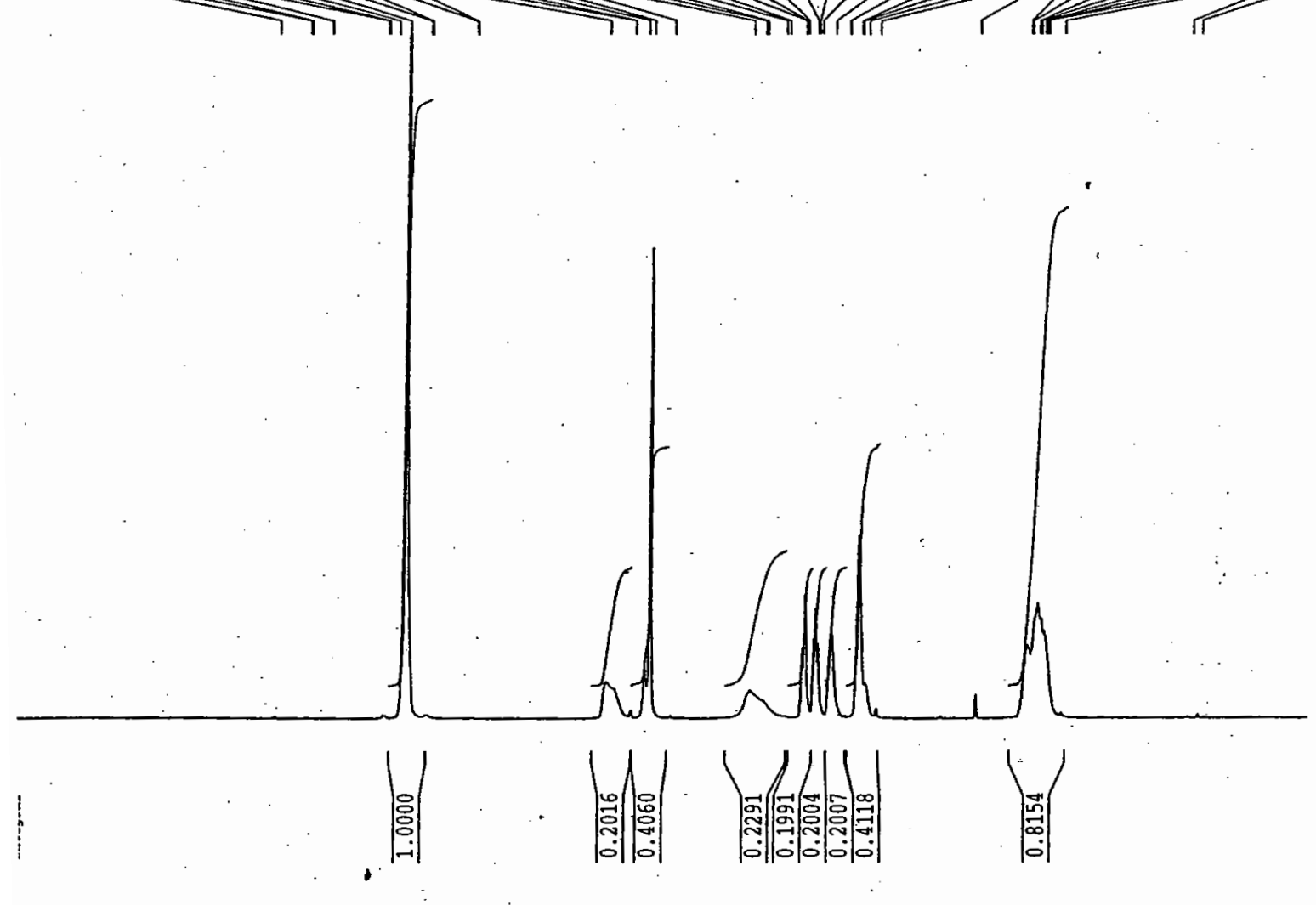

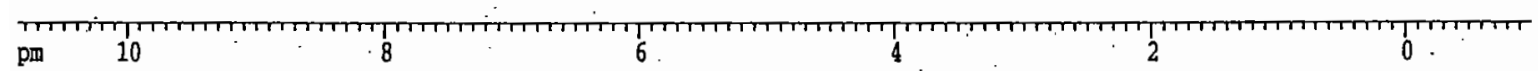

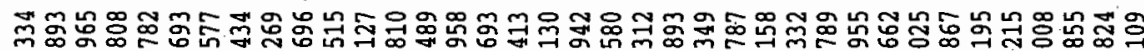

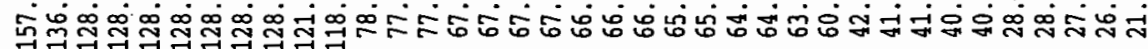
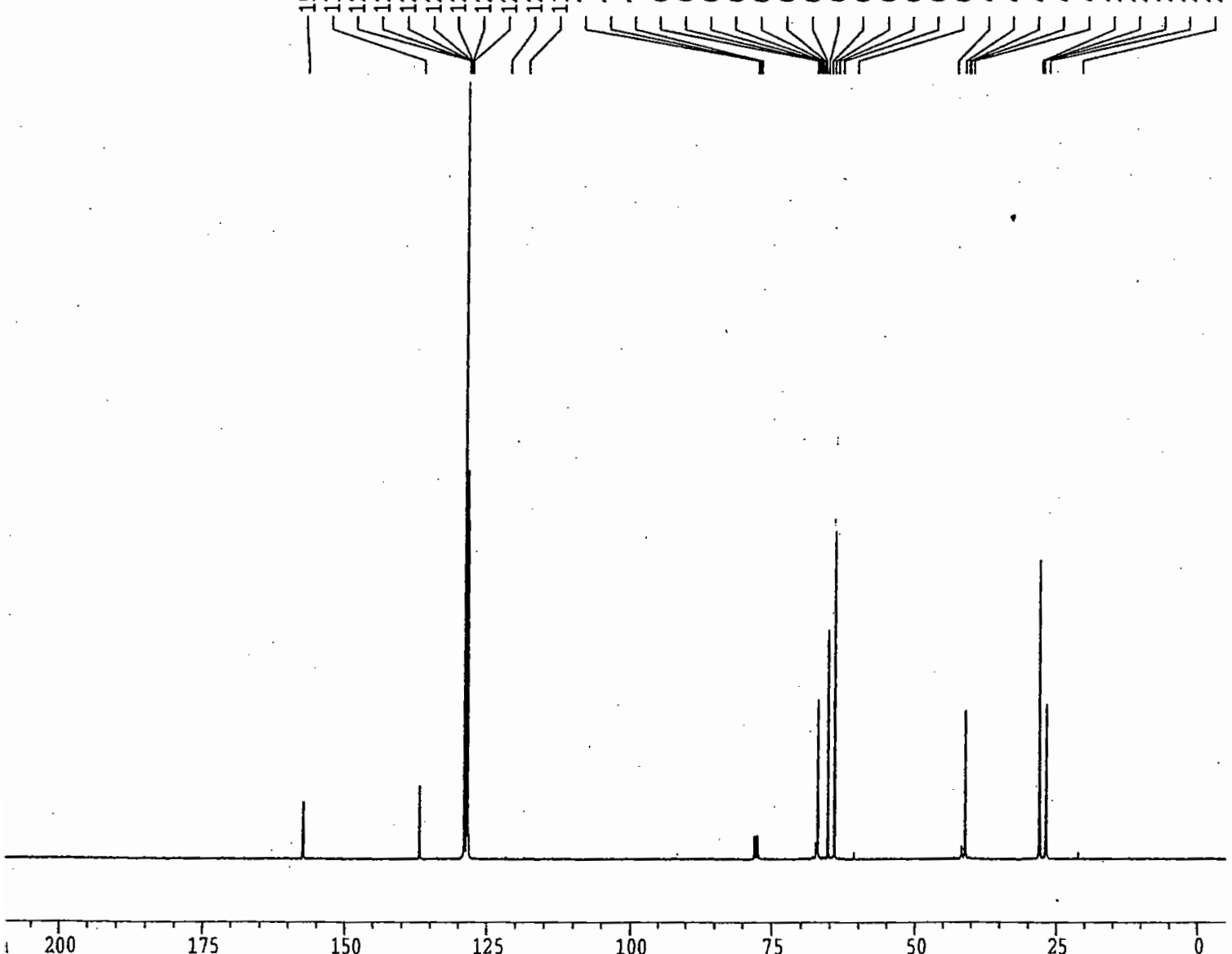


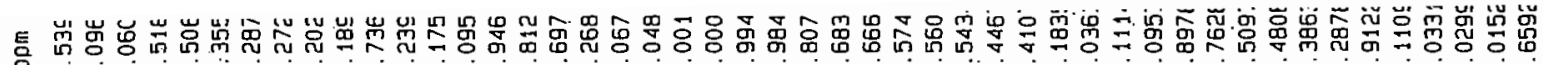
L

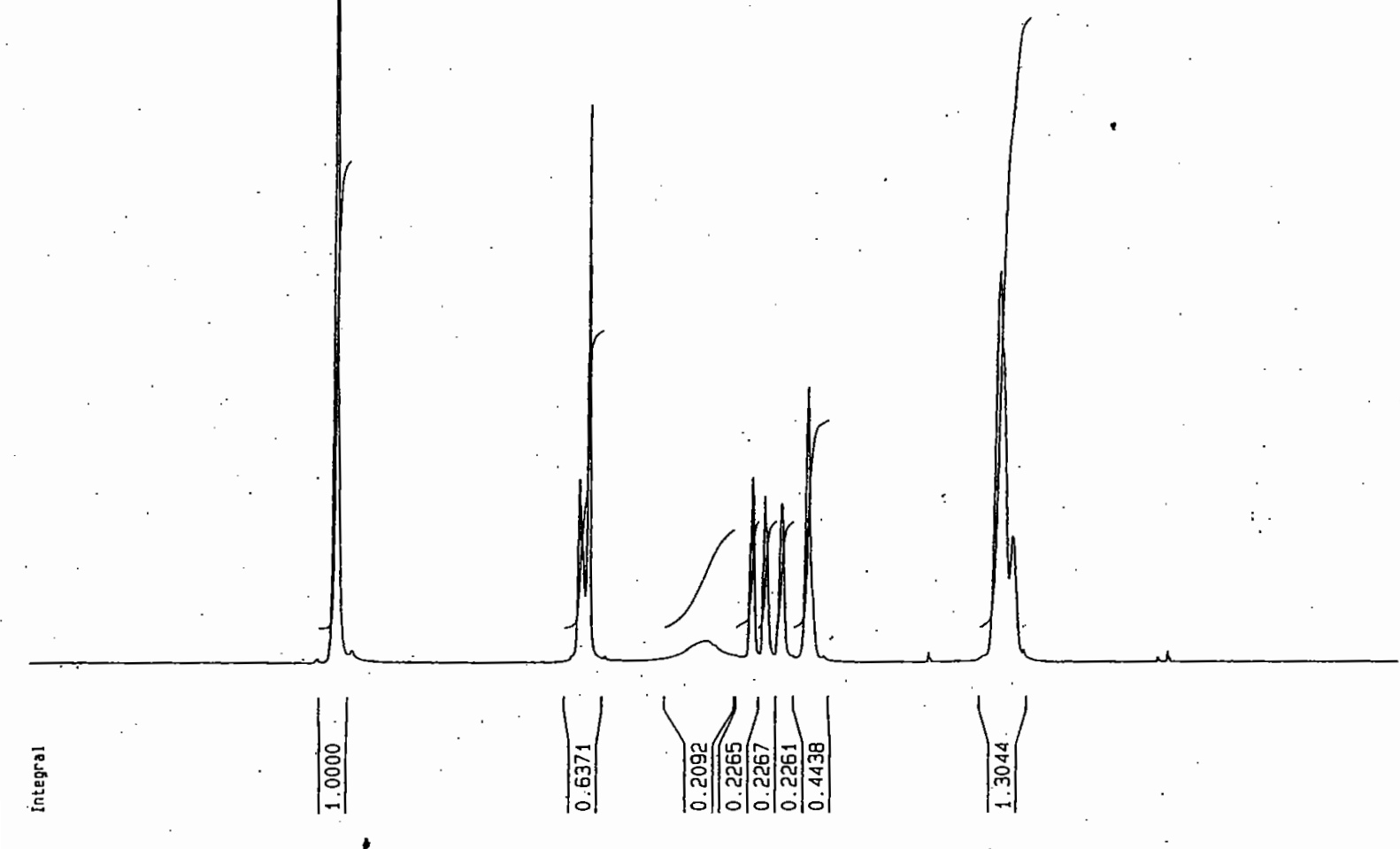

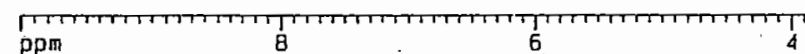

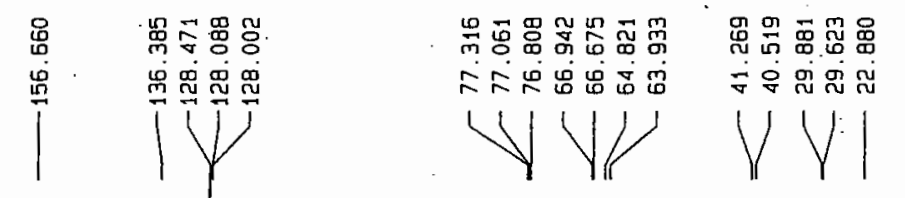

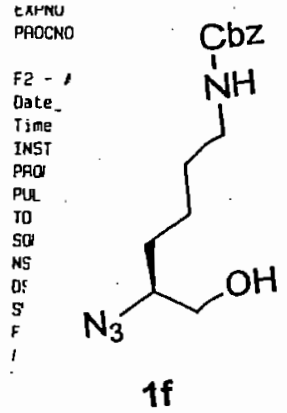

ot
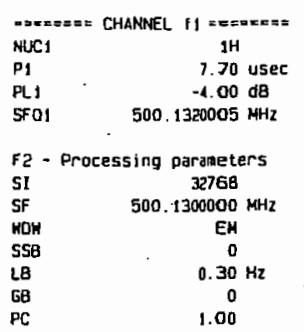

Fi- Processing parneters

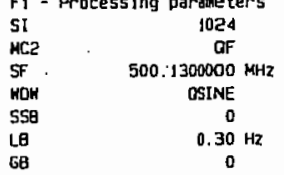

10 NMR plot parameters

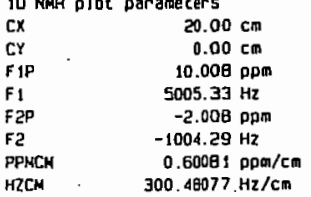

equ

F2 - Acquisstion Parageters

Date 20031229

The

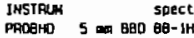

PUPFIS $\quad 209090$

To

solve

NS

sint

FIO
RO
RG

${ }_{10}$
$\mathrm{RG}$
$\mathrm{OW}$
$\mathrm{DE}$
$\mathrm{DE}$
$\mathrm{TE}$

$0 \mathrm{~W}$
$0 \mathrm{E}$
$\mathrm{TE}$
01
011
$d 1$

d12

$$
\begin{aligned}
& \text { PUC } \\
& P 1 \\
& P 1 \\
& \text { PFo }
\end{aligned}
$$

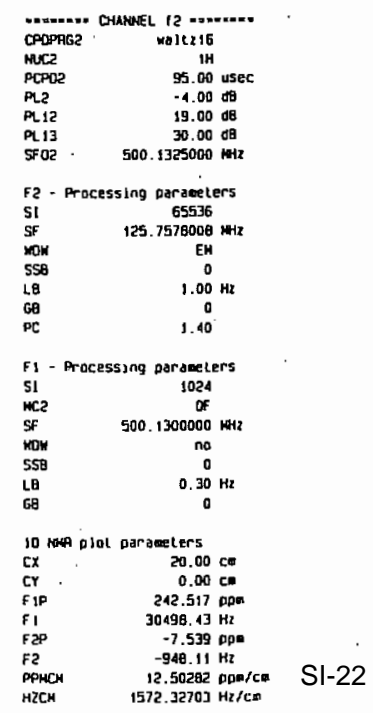



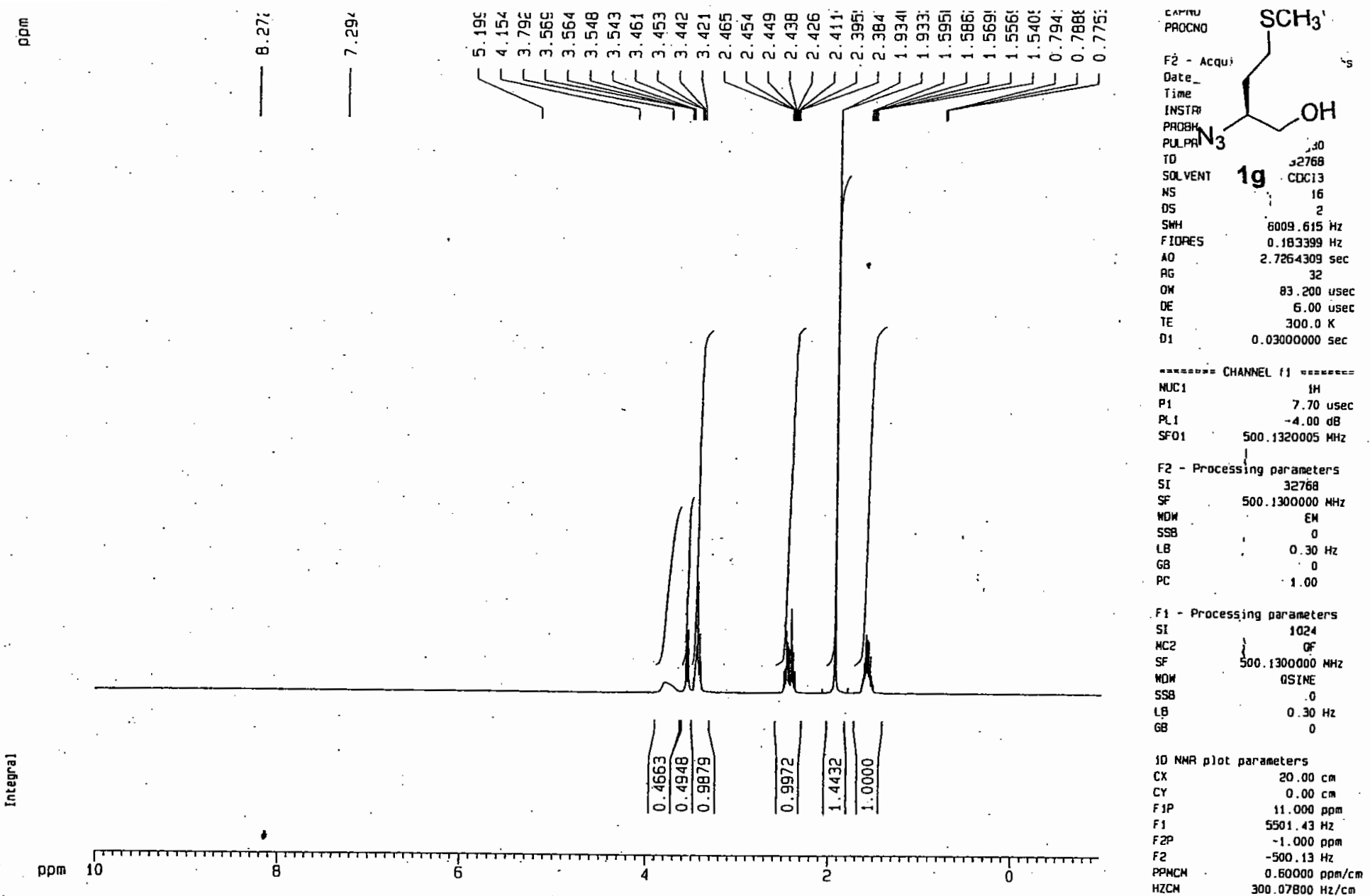

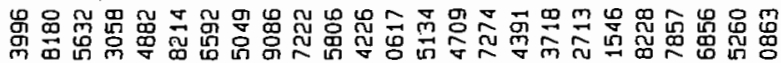

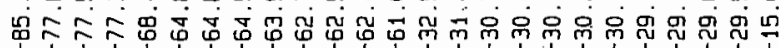

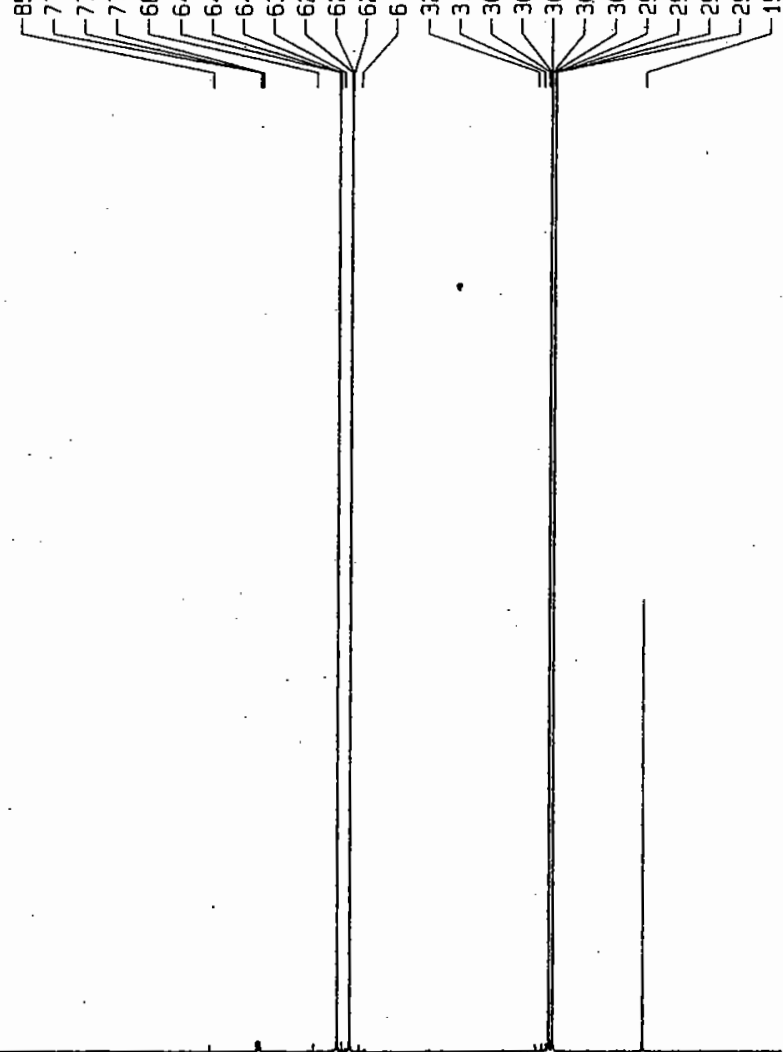




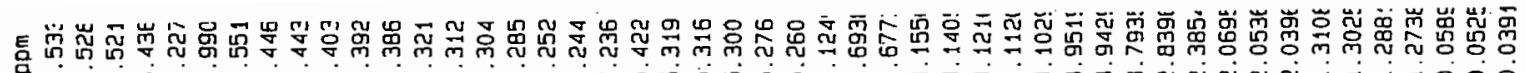

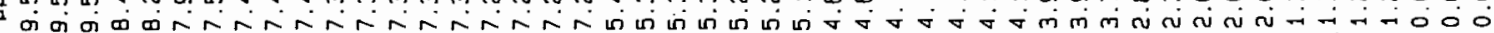
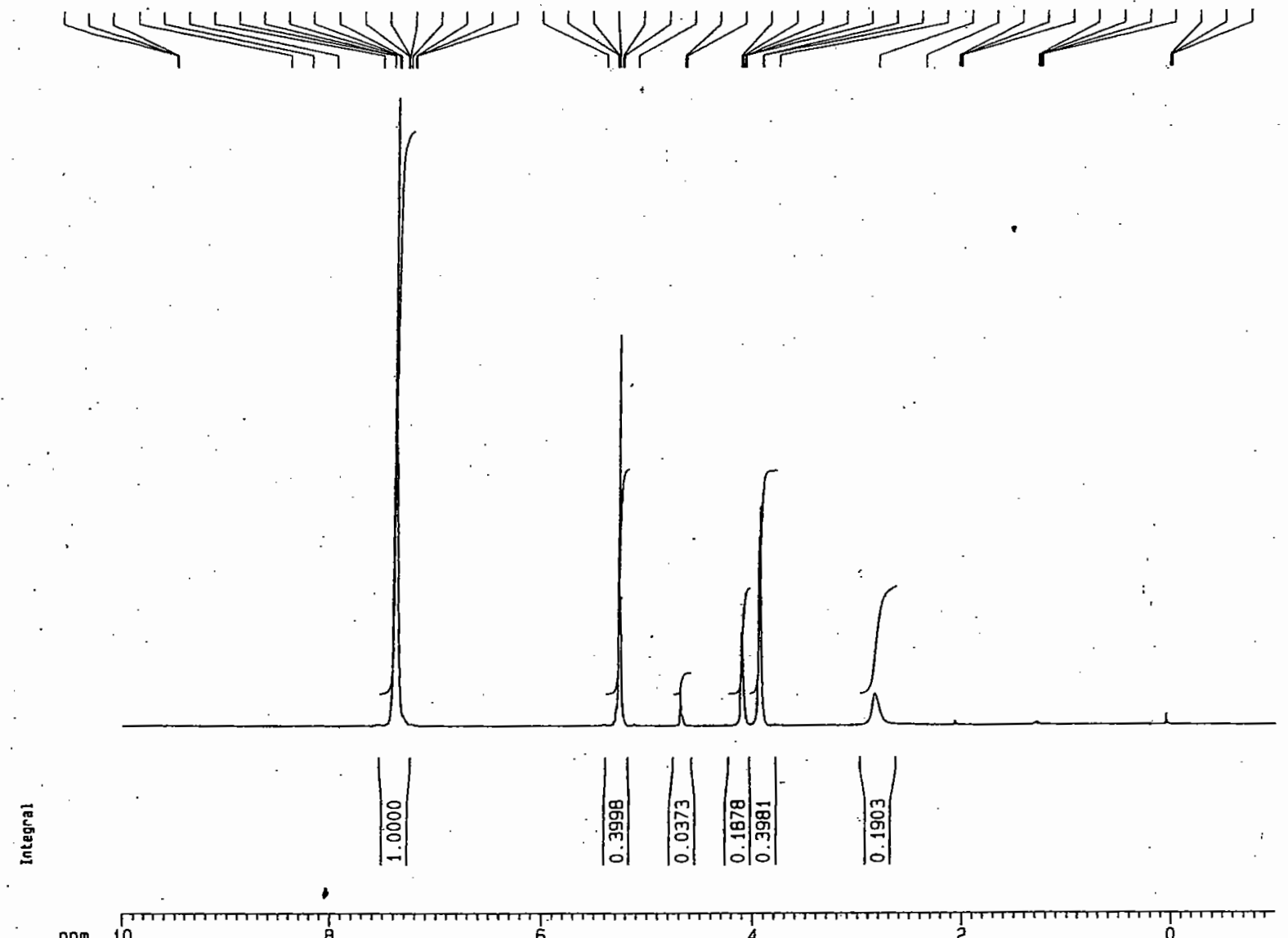<smiles>N[C@H](CO)C(=O)OCc1ccccc1</smiles>

1h

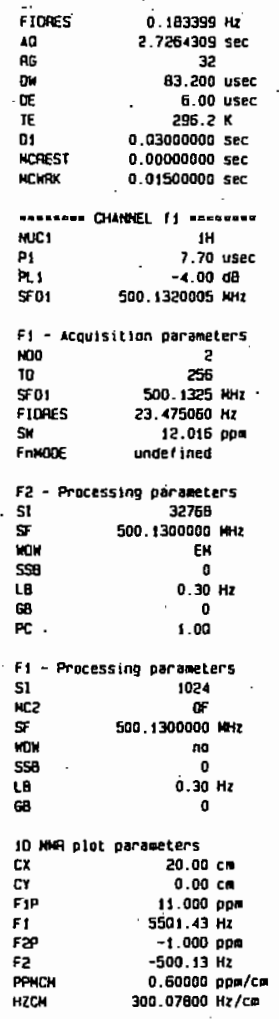

言

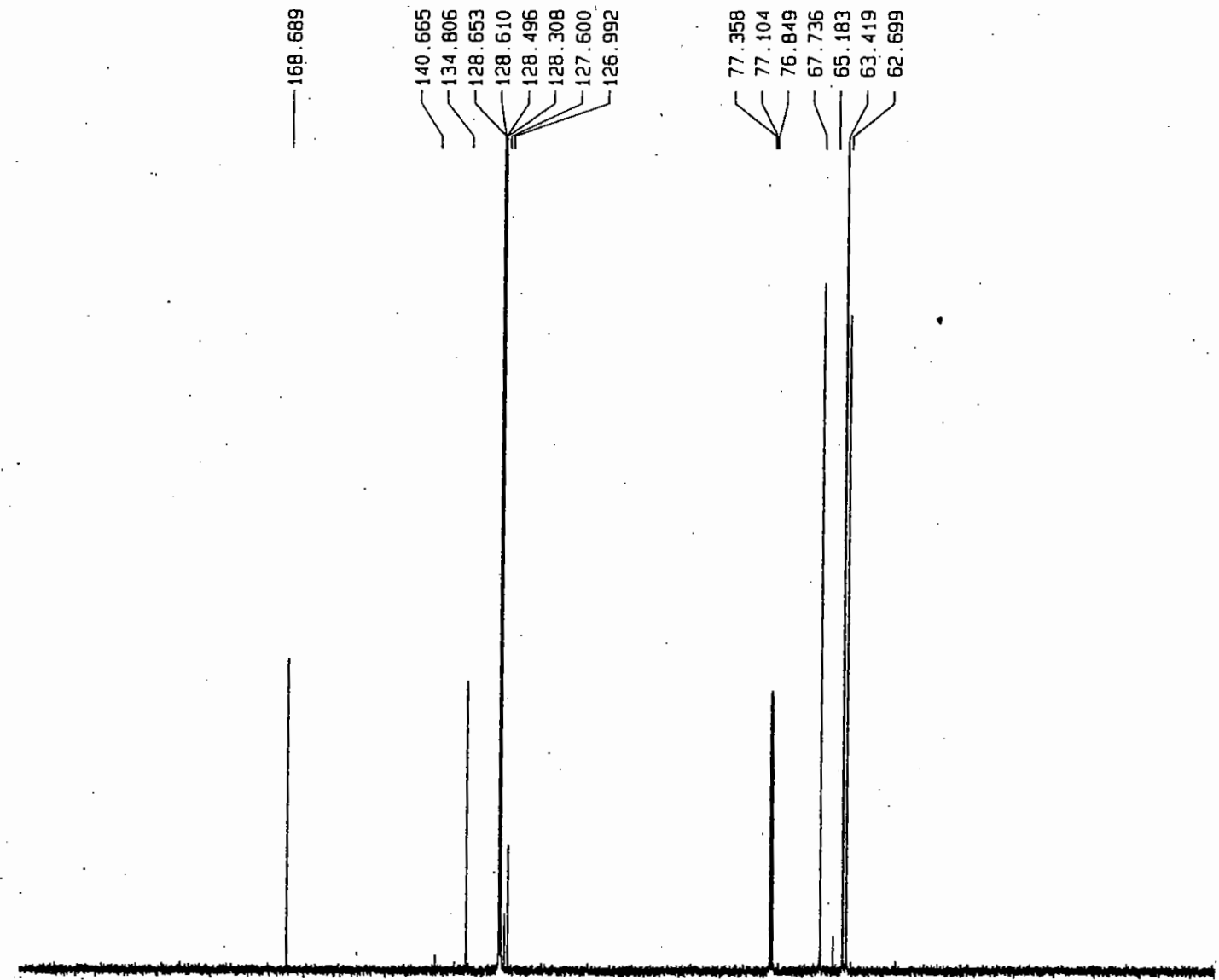

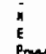

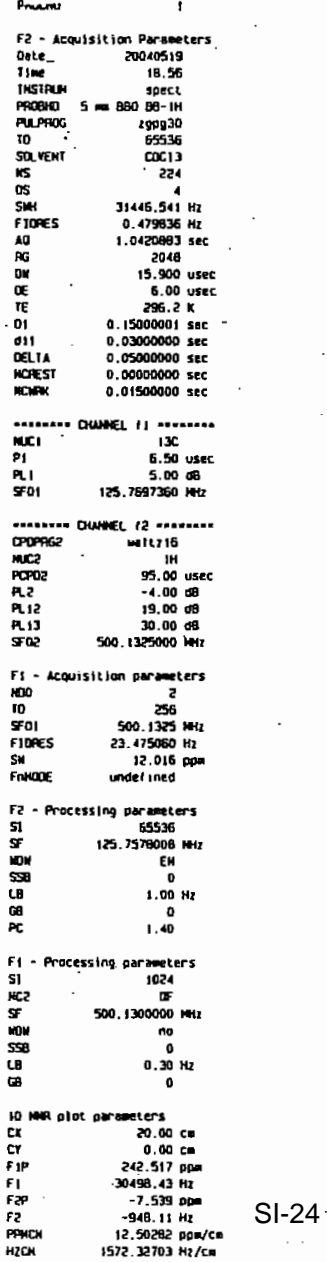



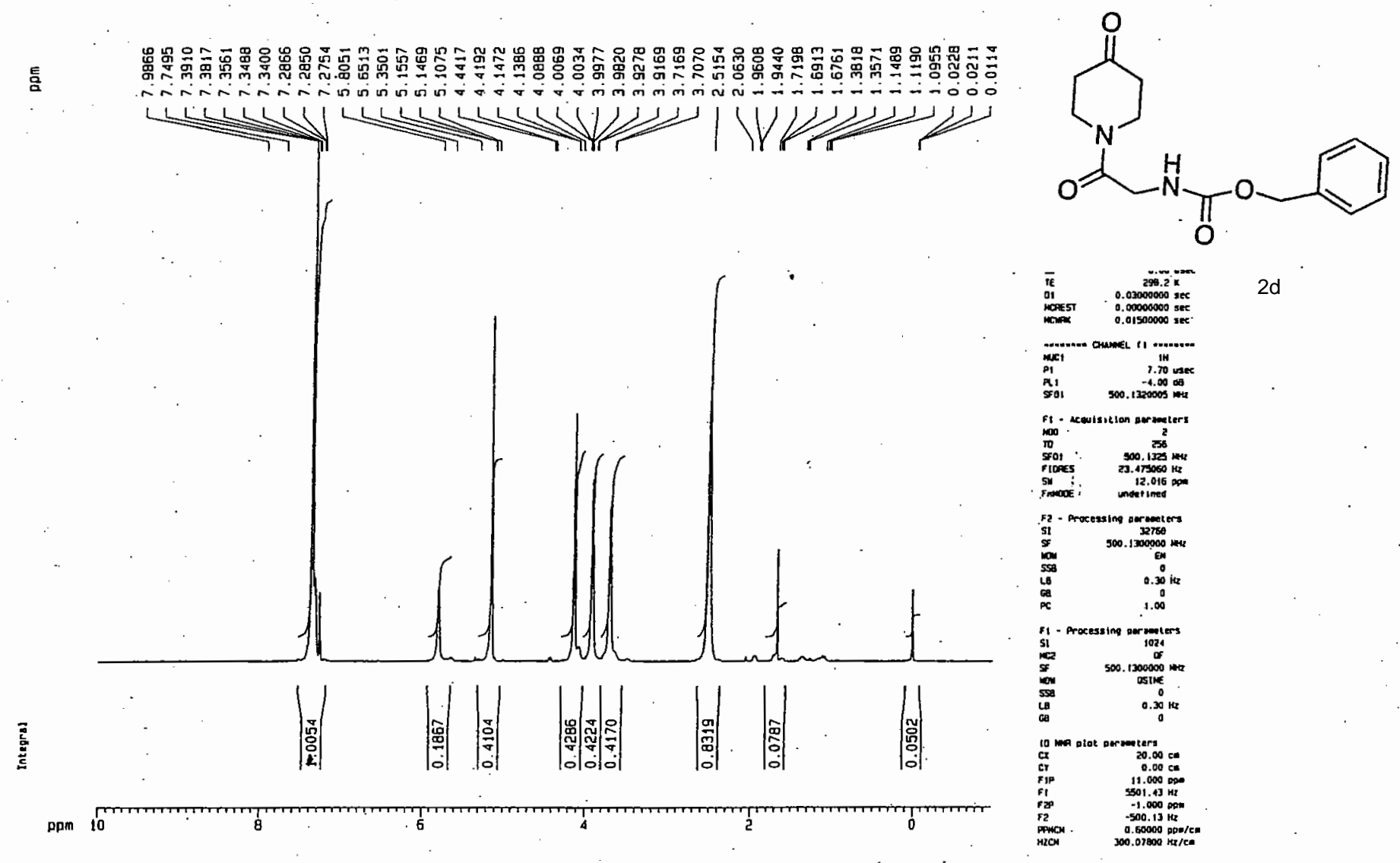

$-4.0060$

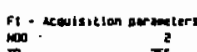

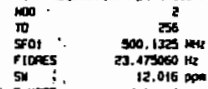

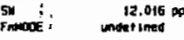

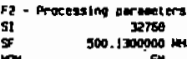

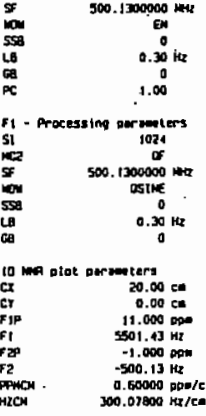

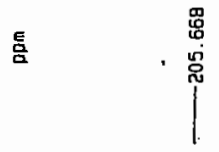
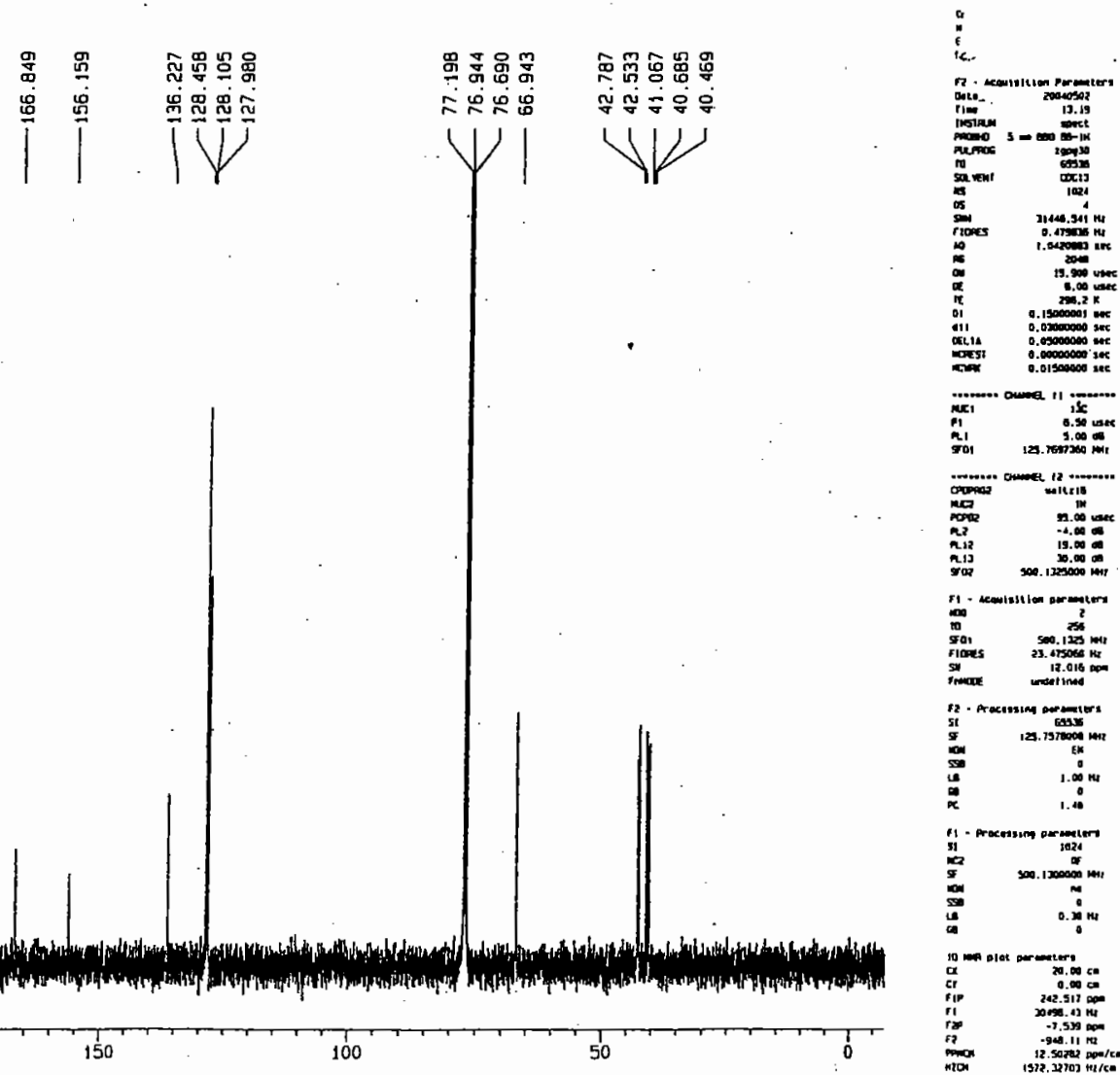


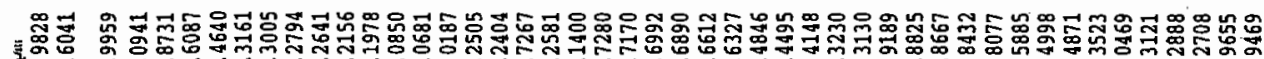

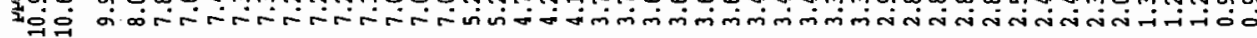

j) 1 (L)
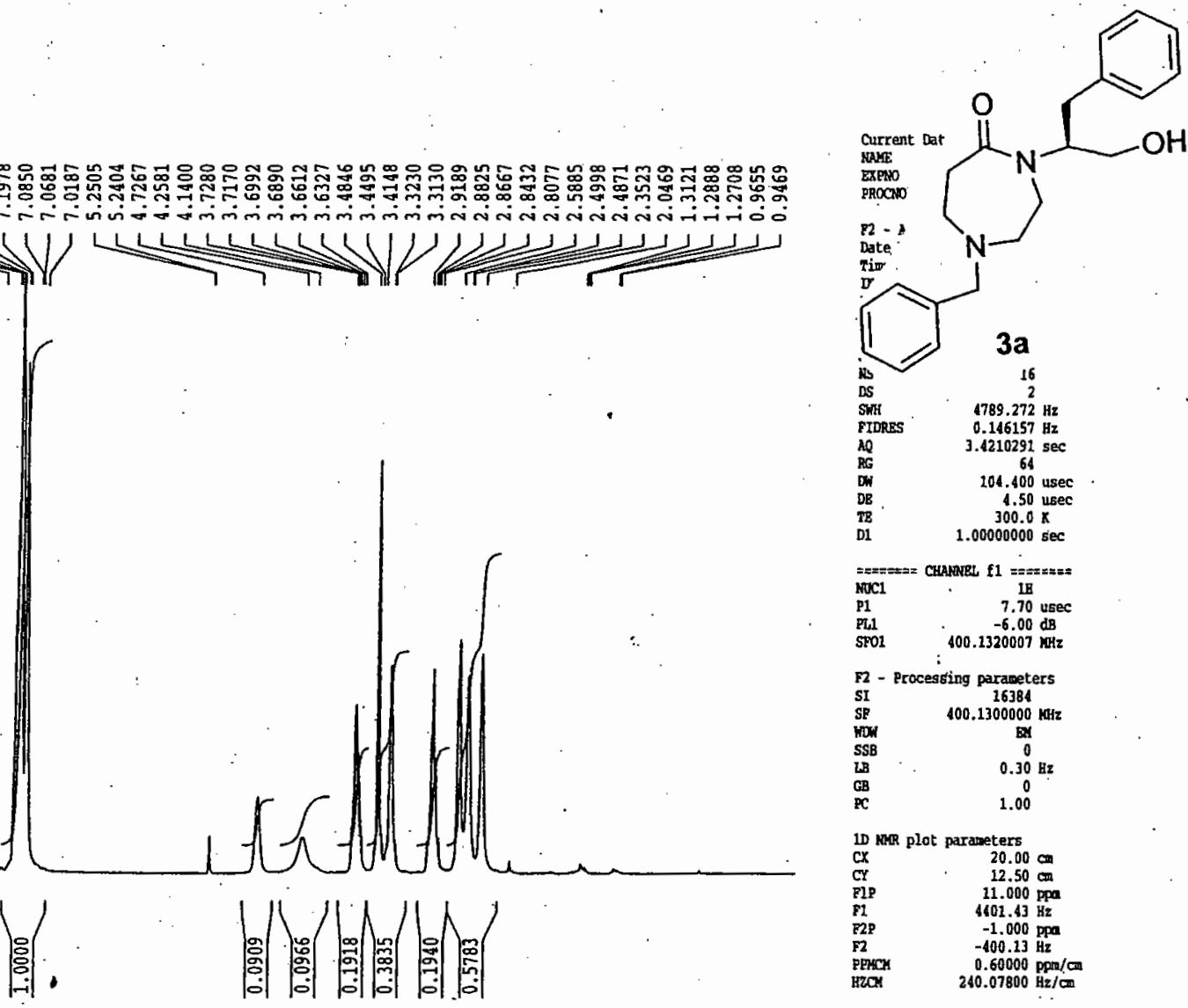

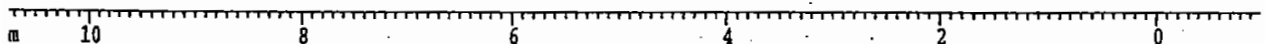
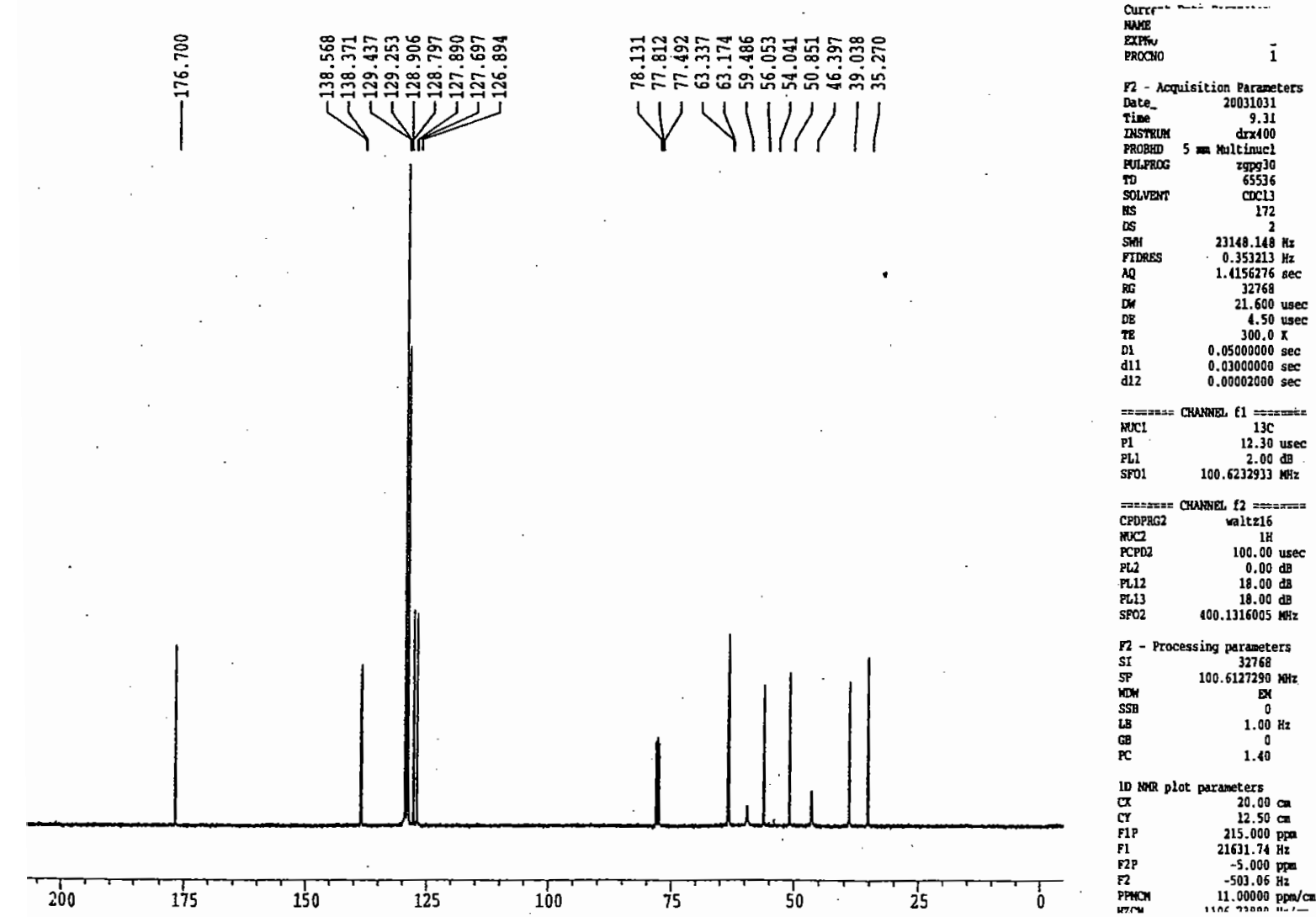


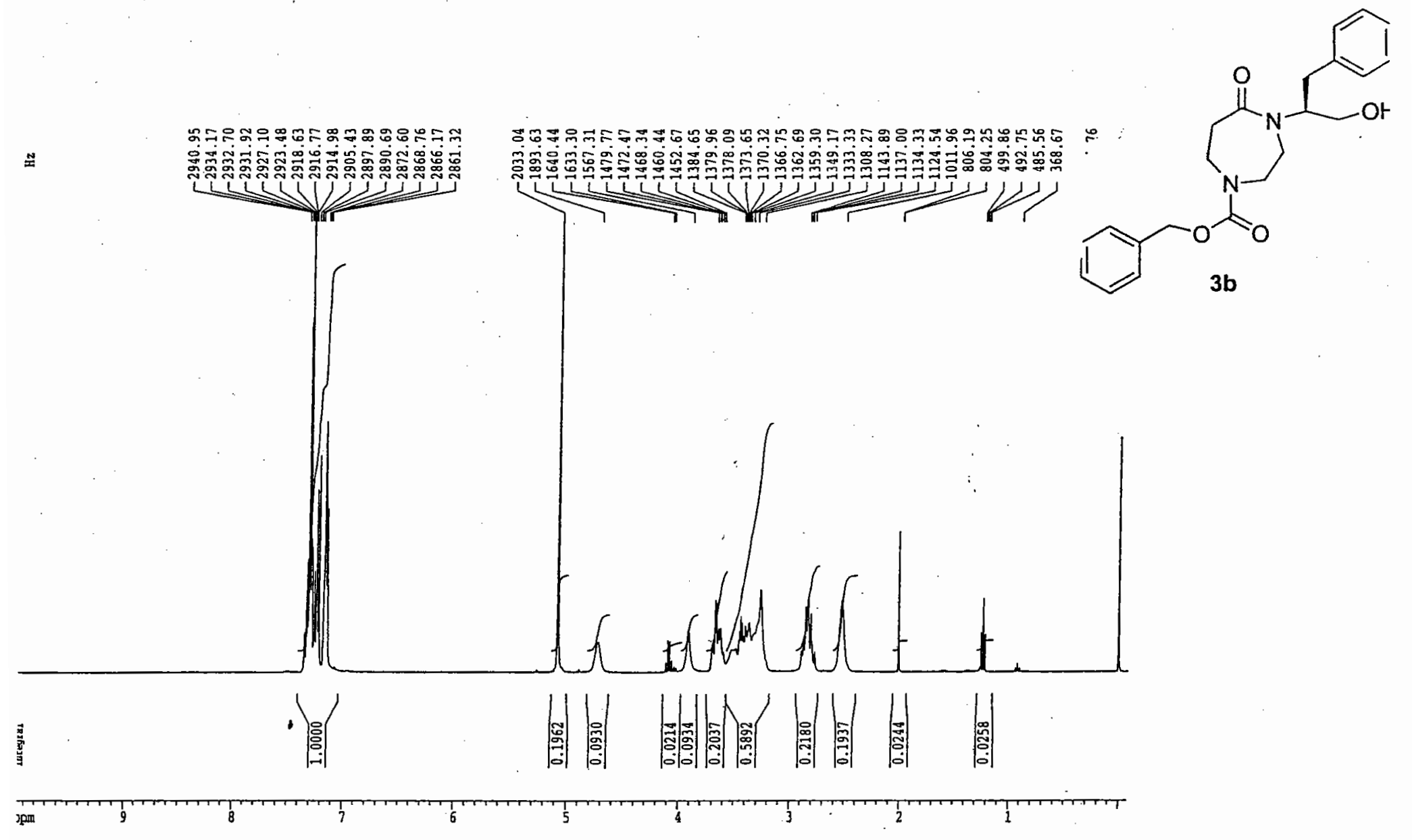

V.



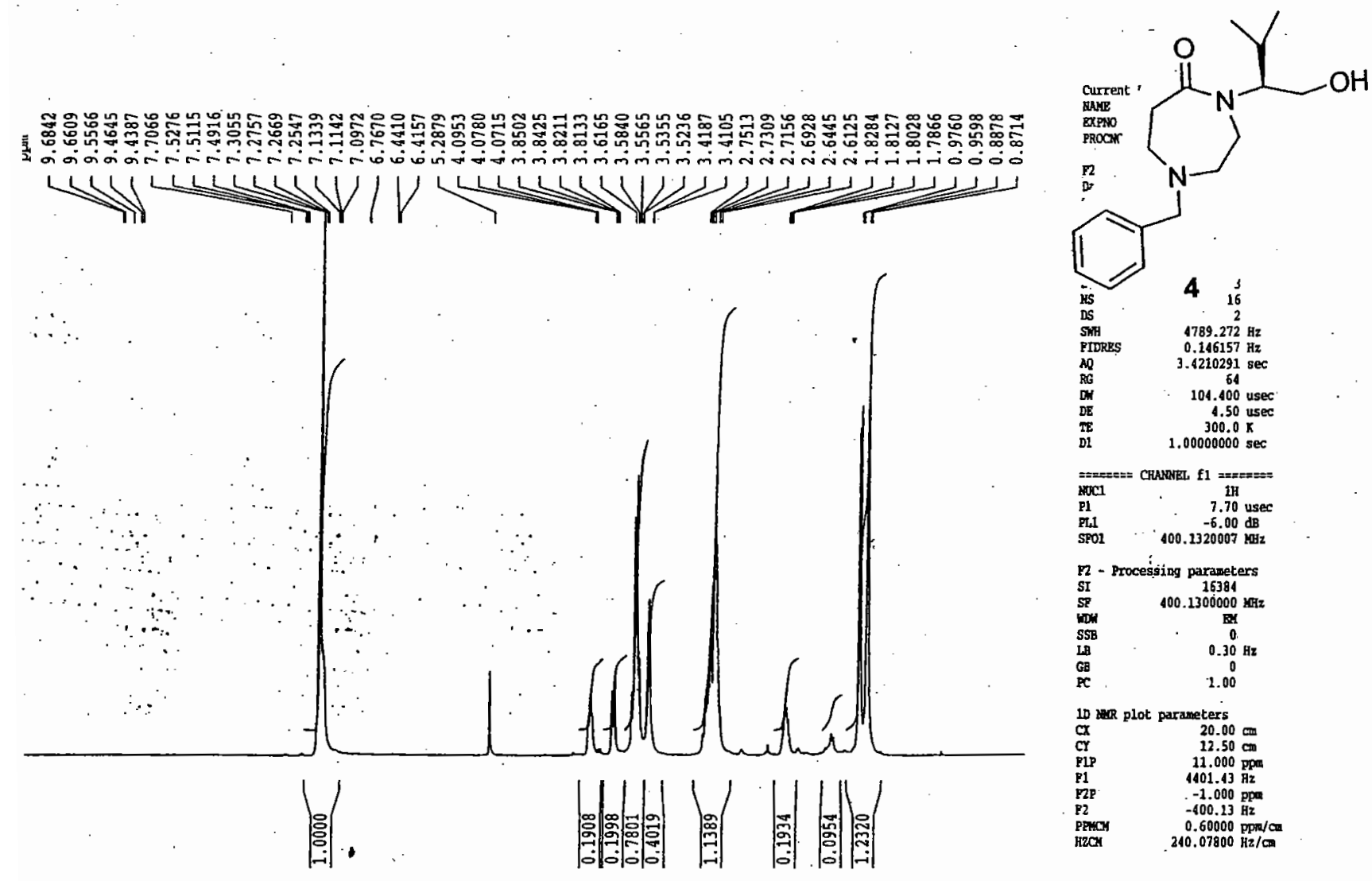

II 10 .

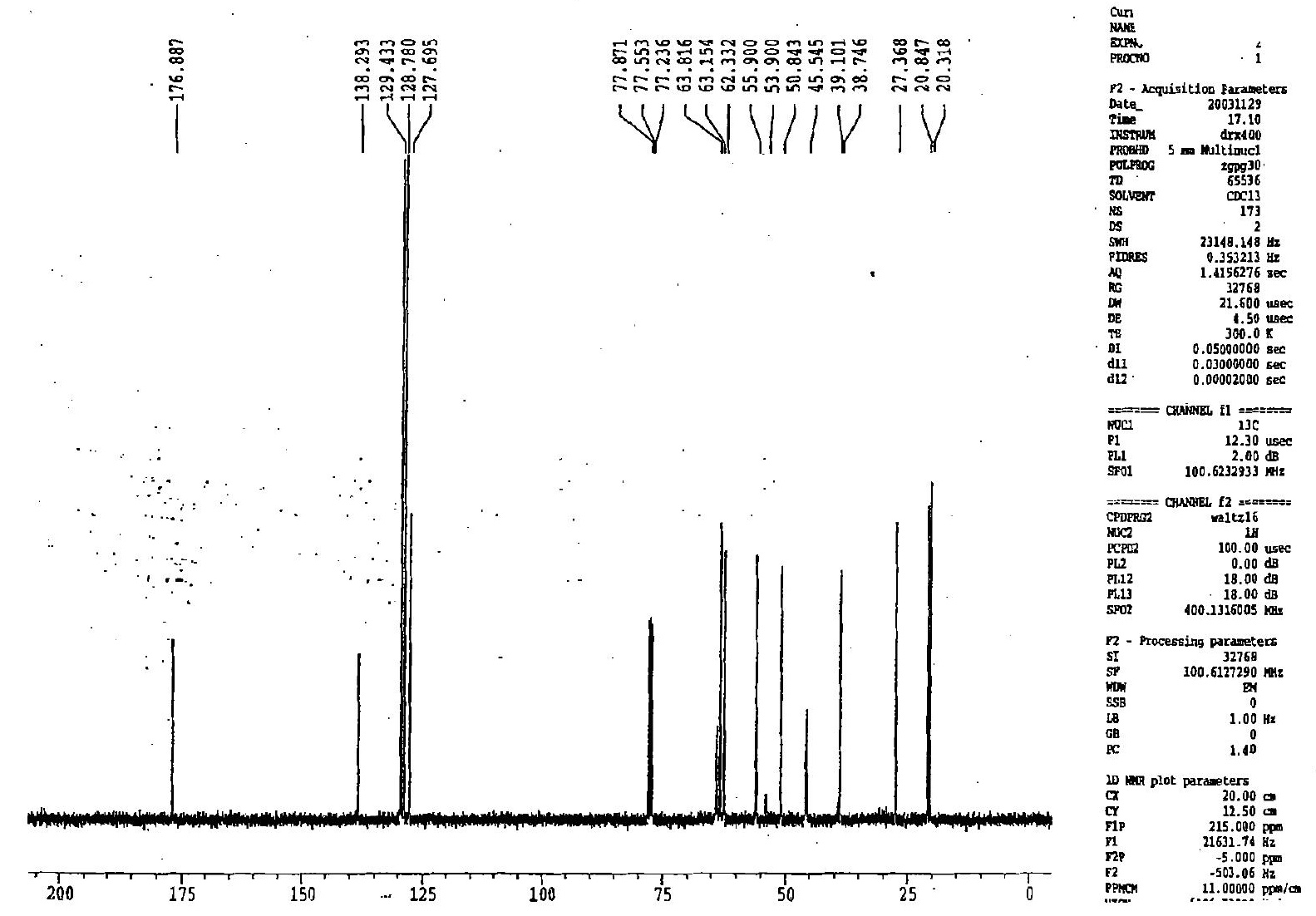



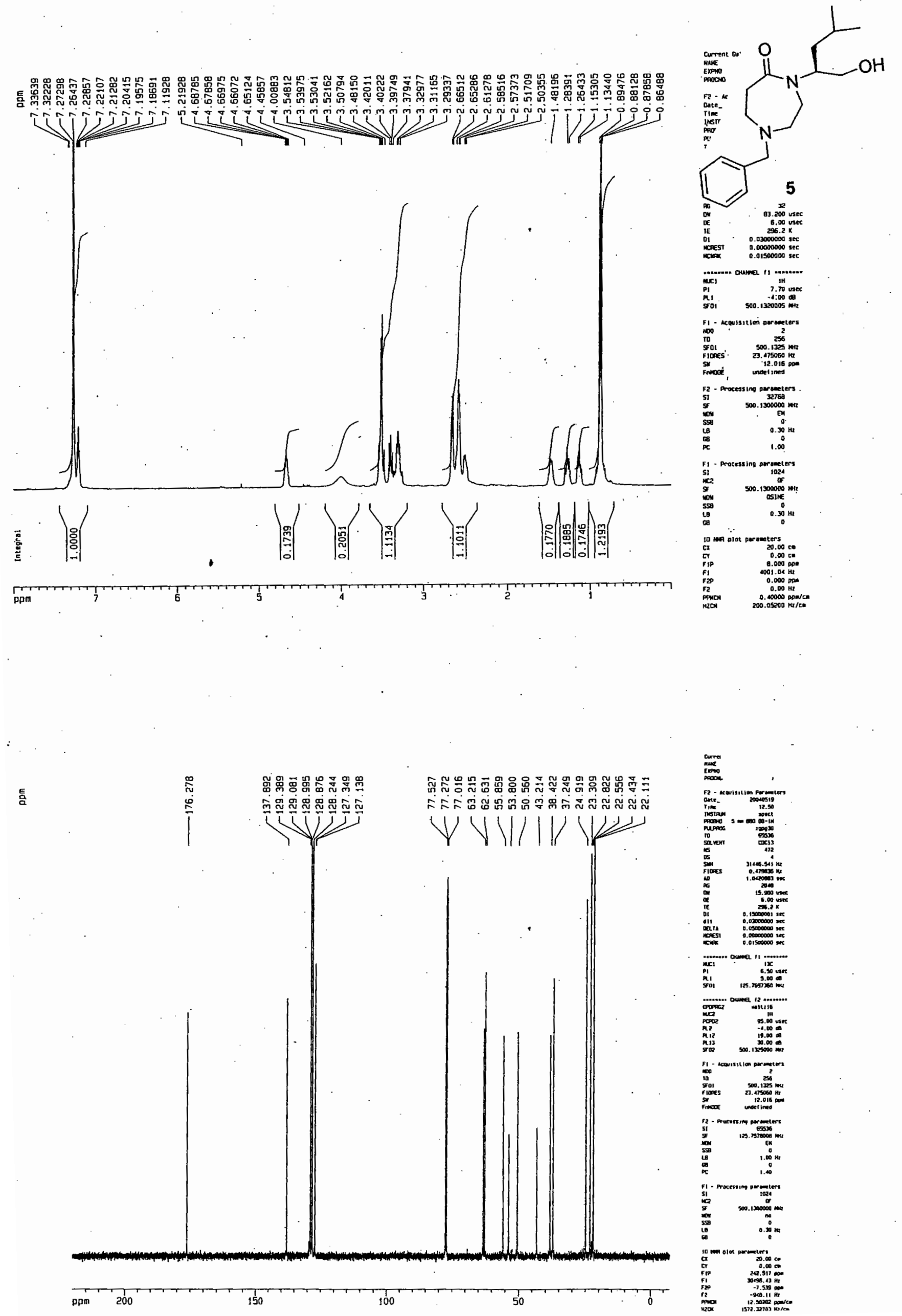

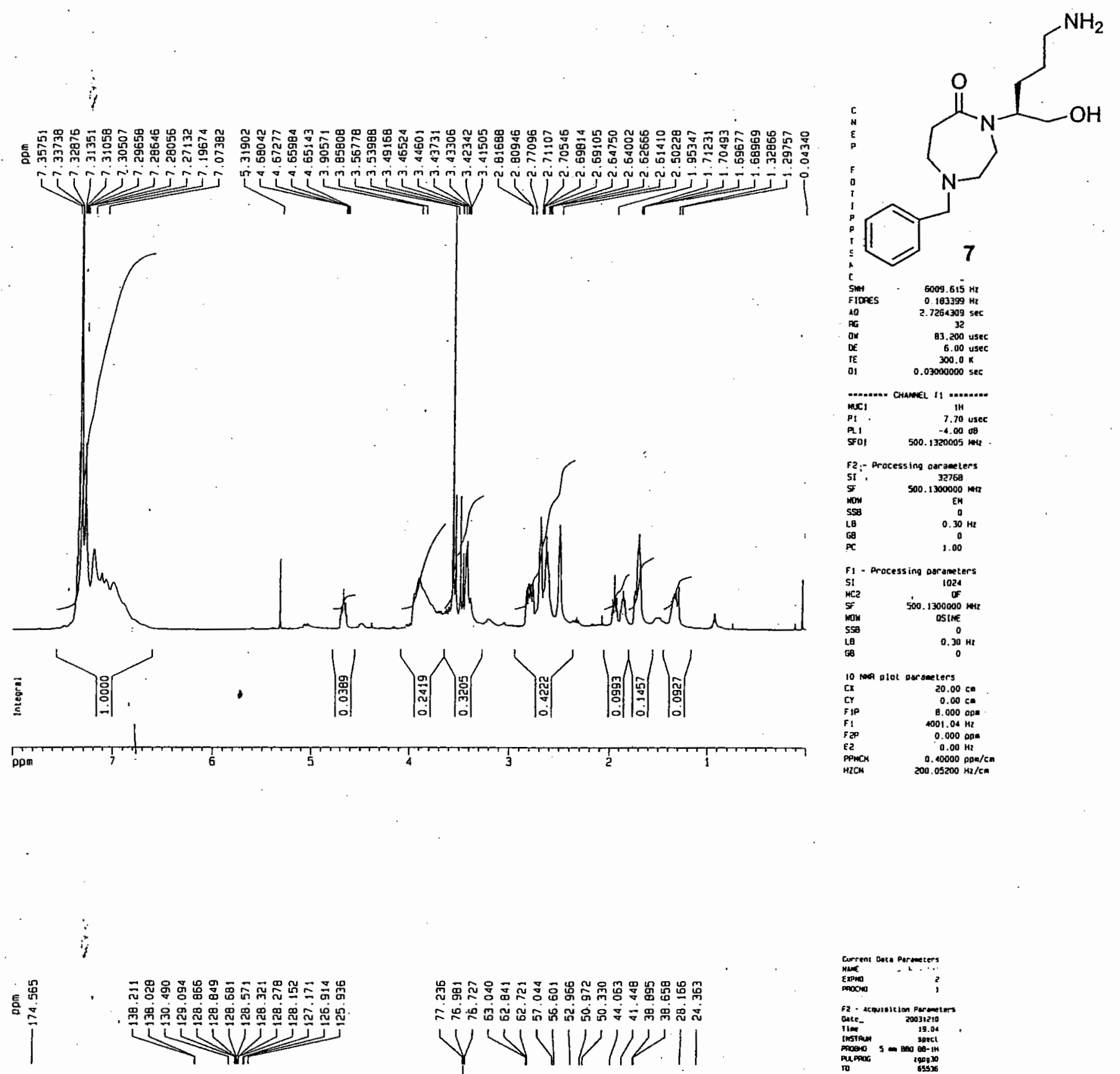

|v

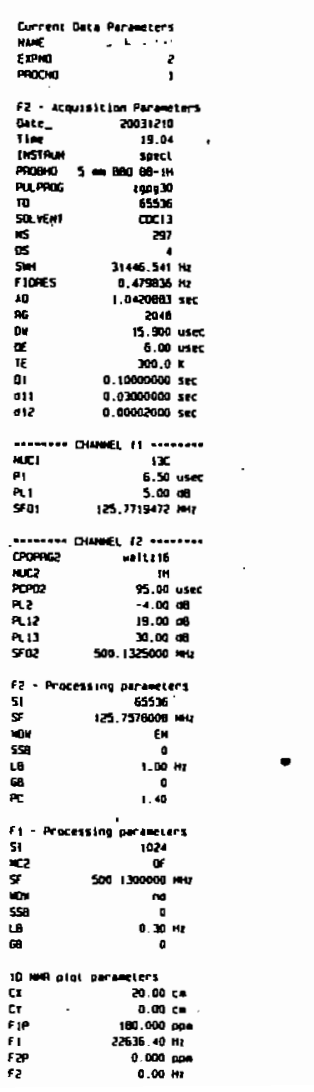



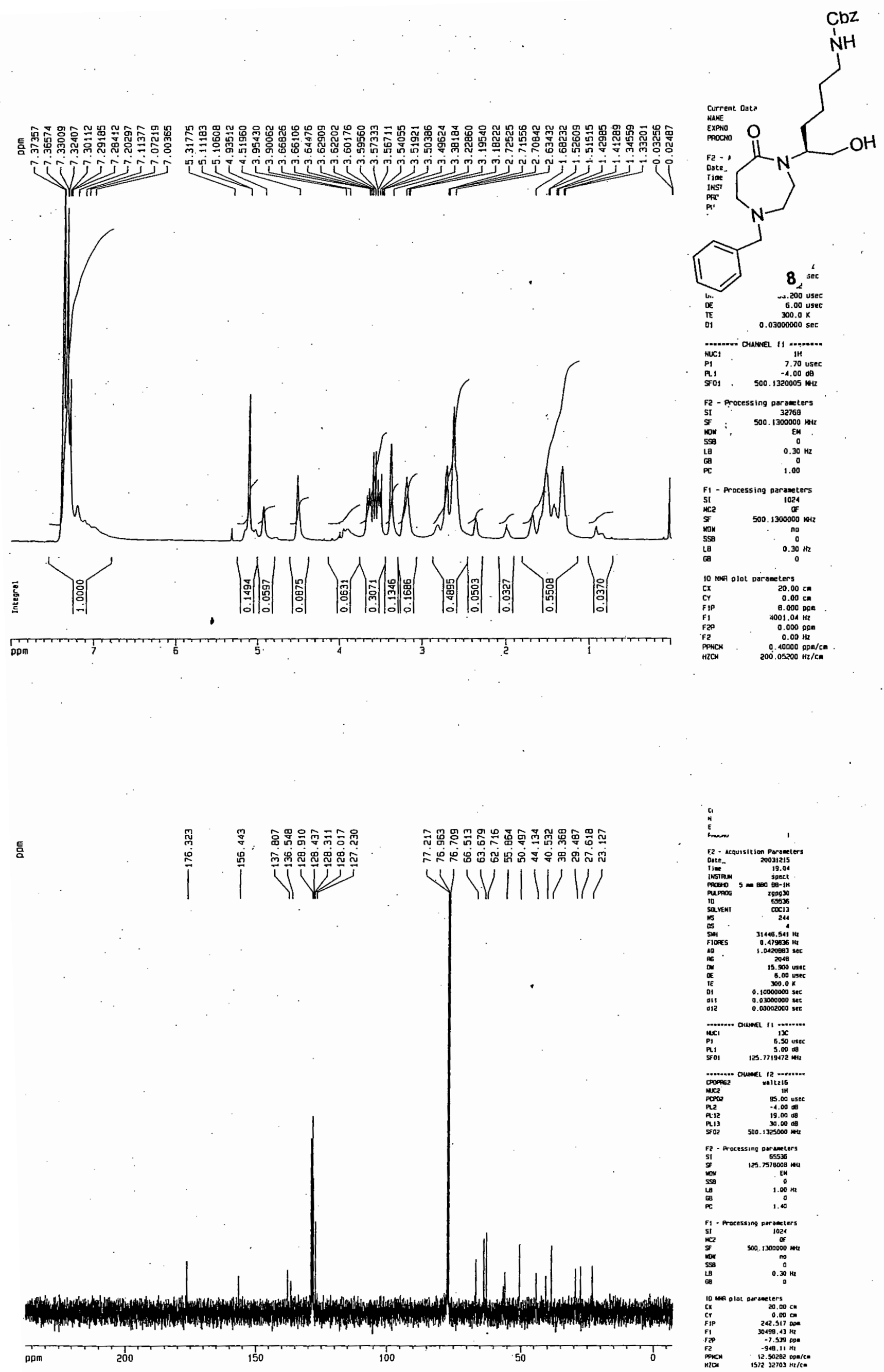

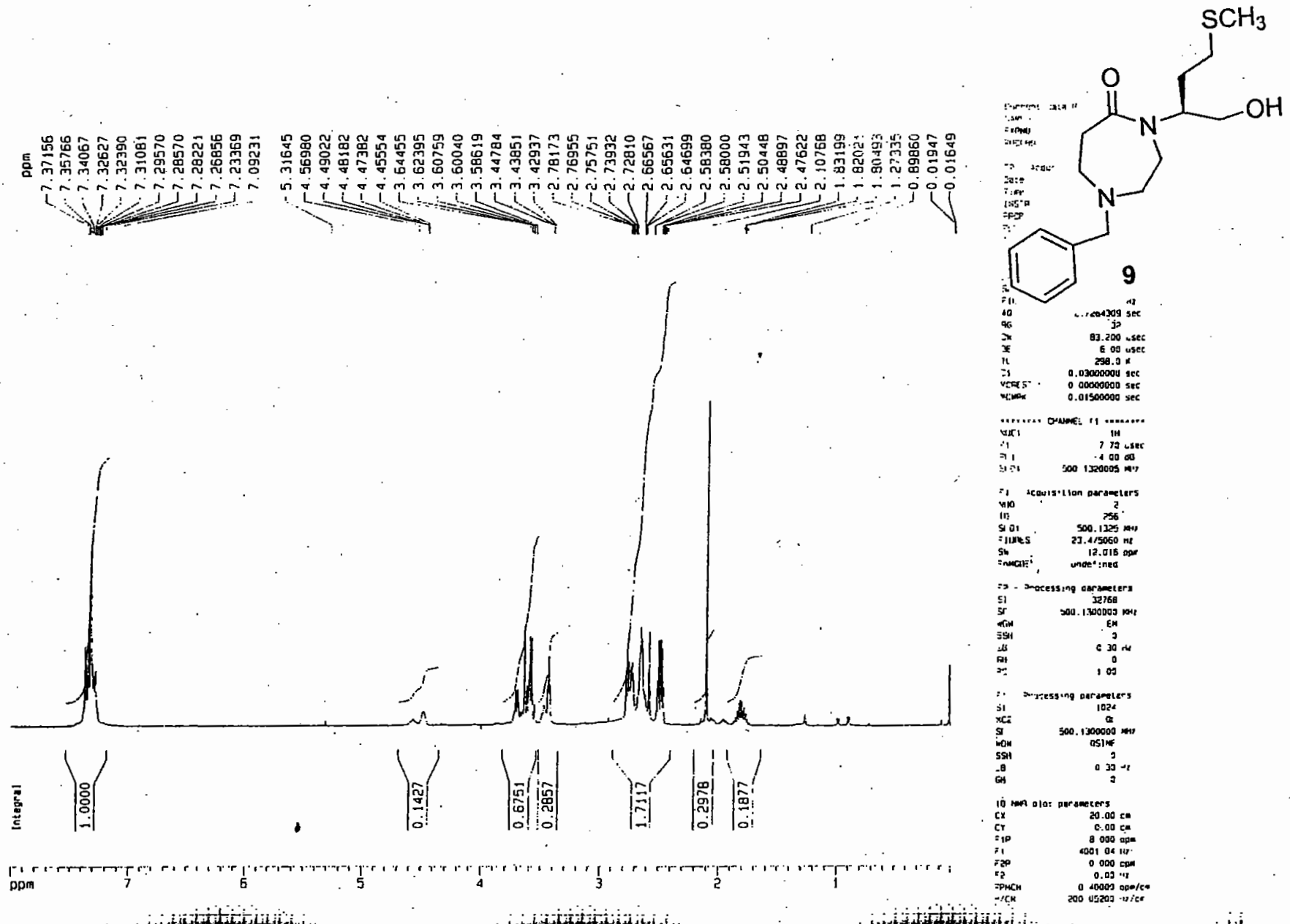

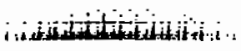

亭

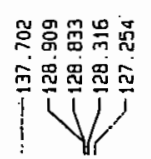

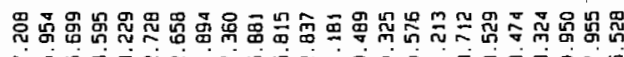

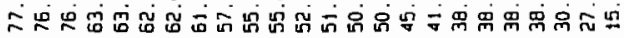
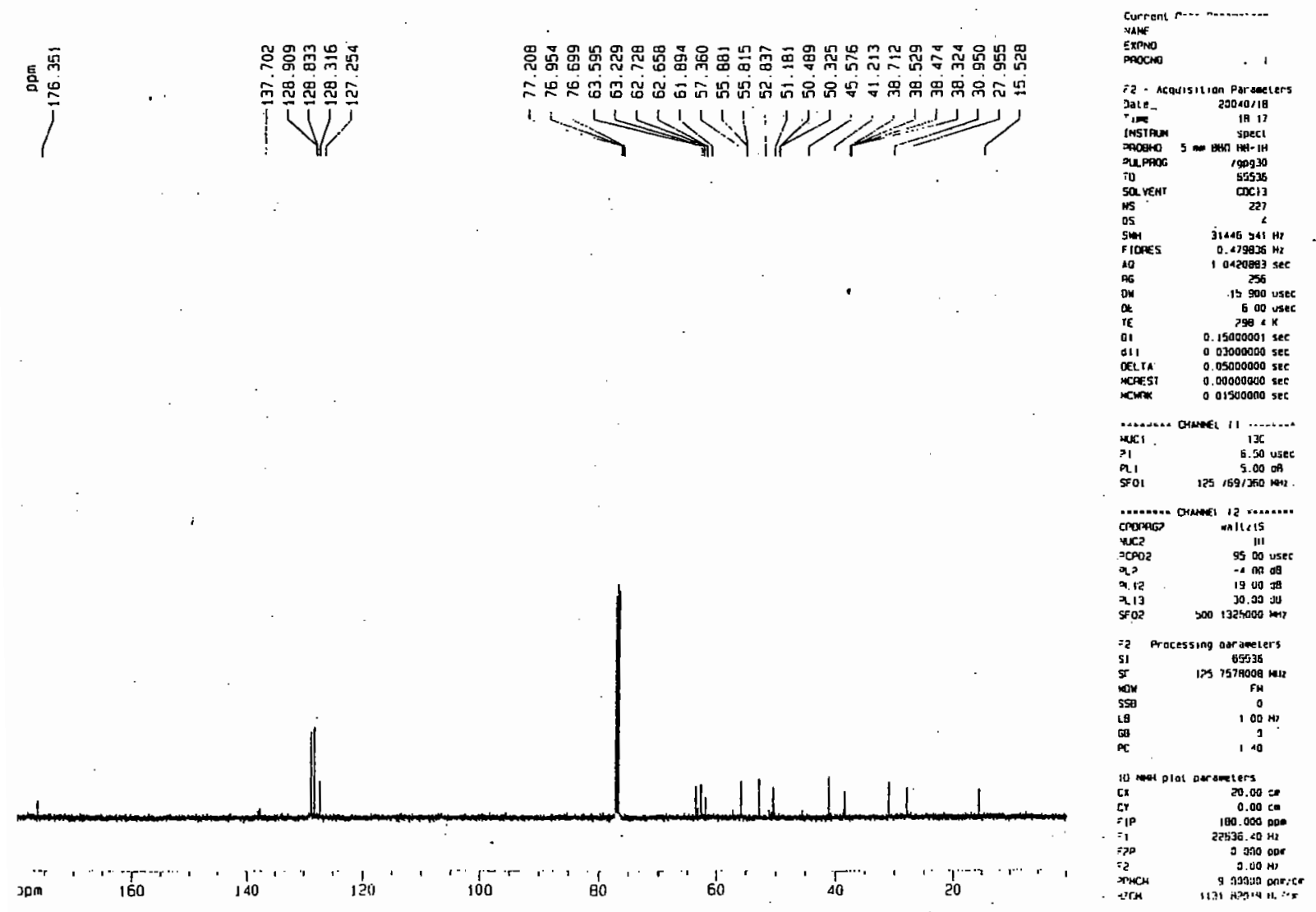

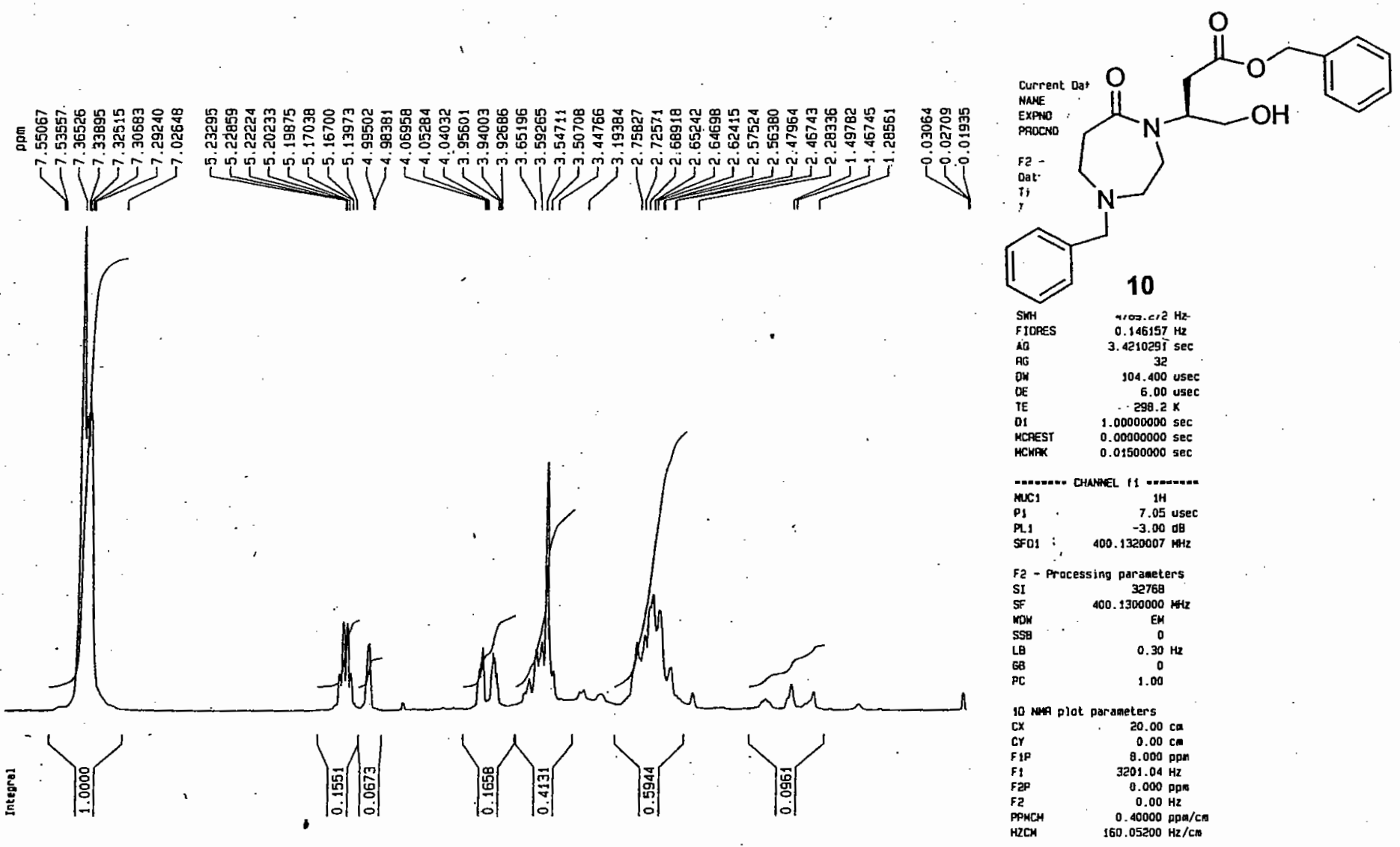
竞

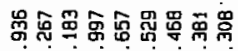
Ens

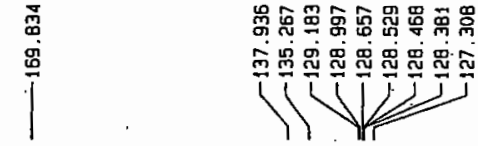

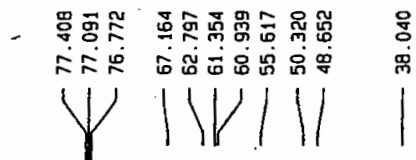

.
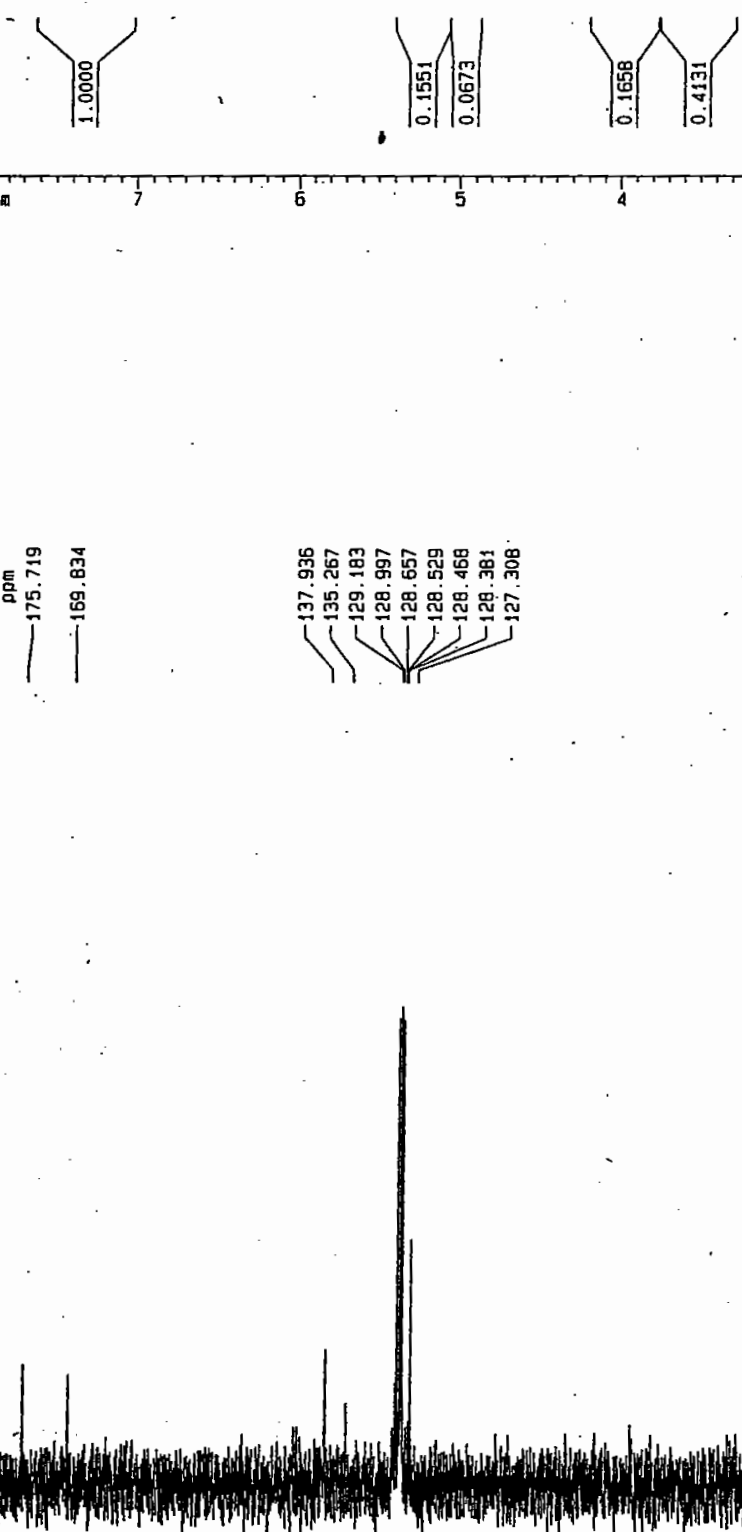

$>$

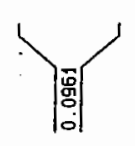

pp

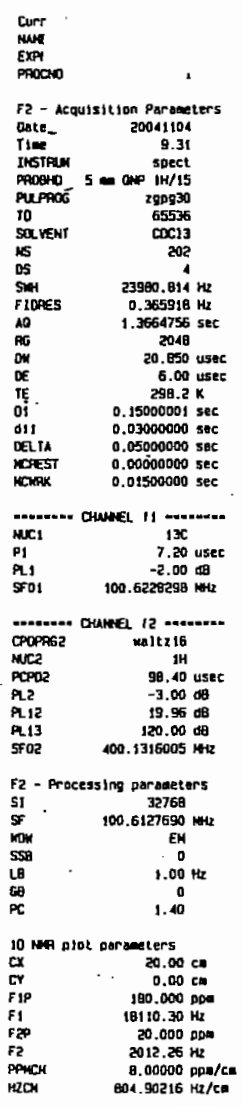


구윰ำ
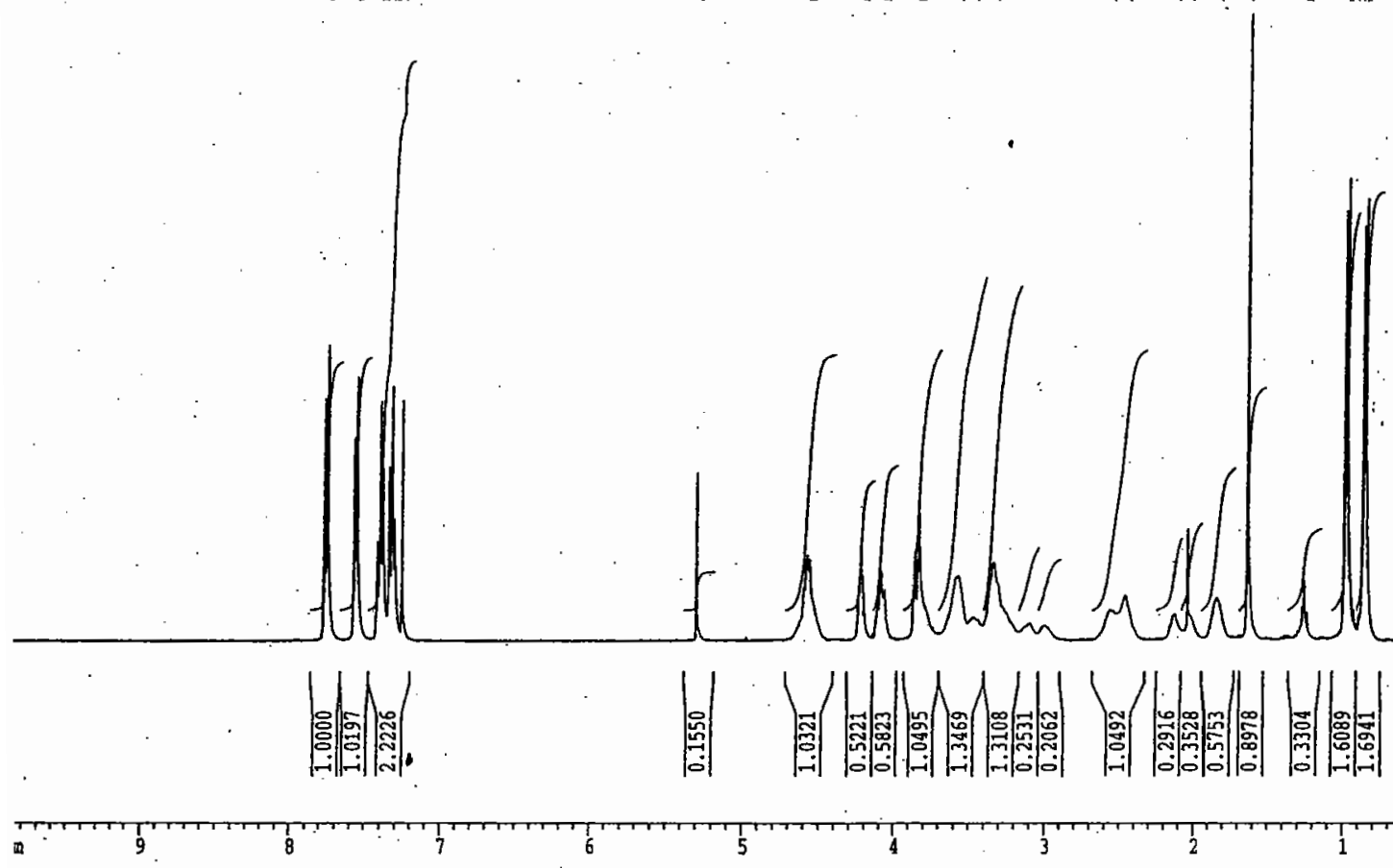

6 Tremert 5
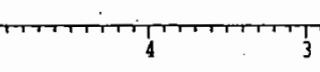

3 गrm?
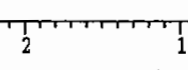

The
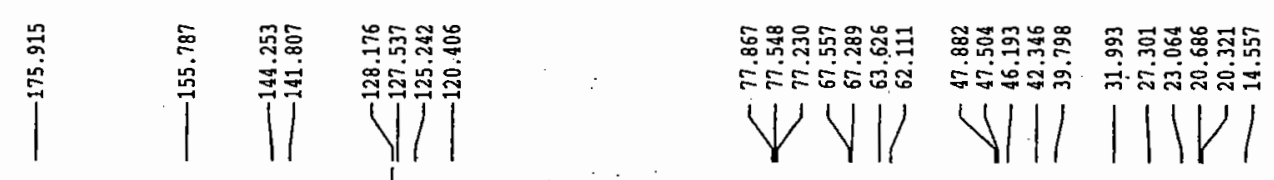

,

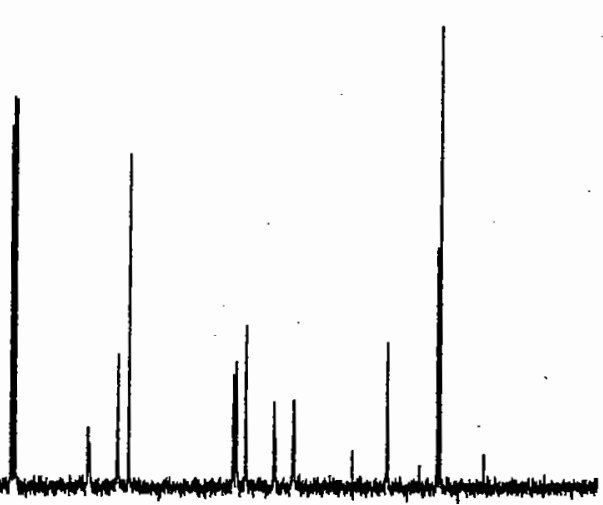



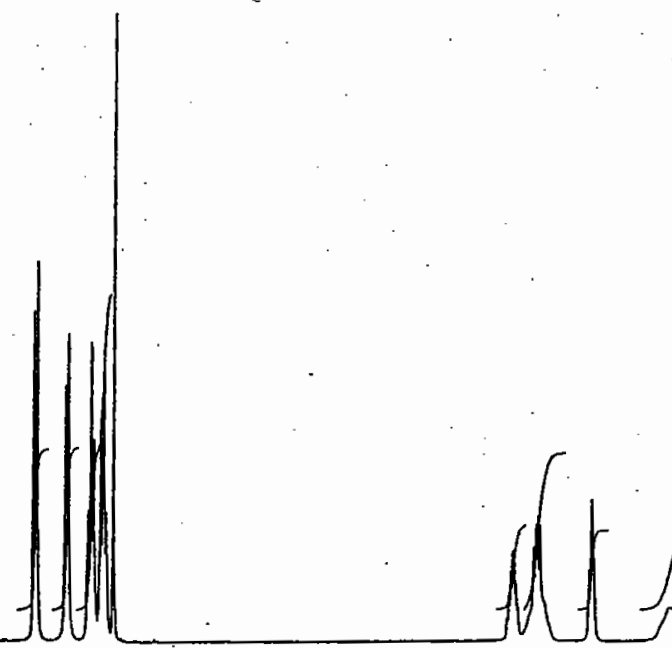<smiles>CCC1c2ccccc2-c2ccccc21</smiles>
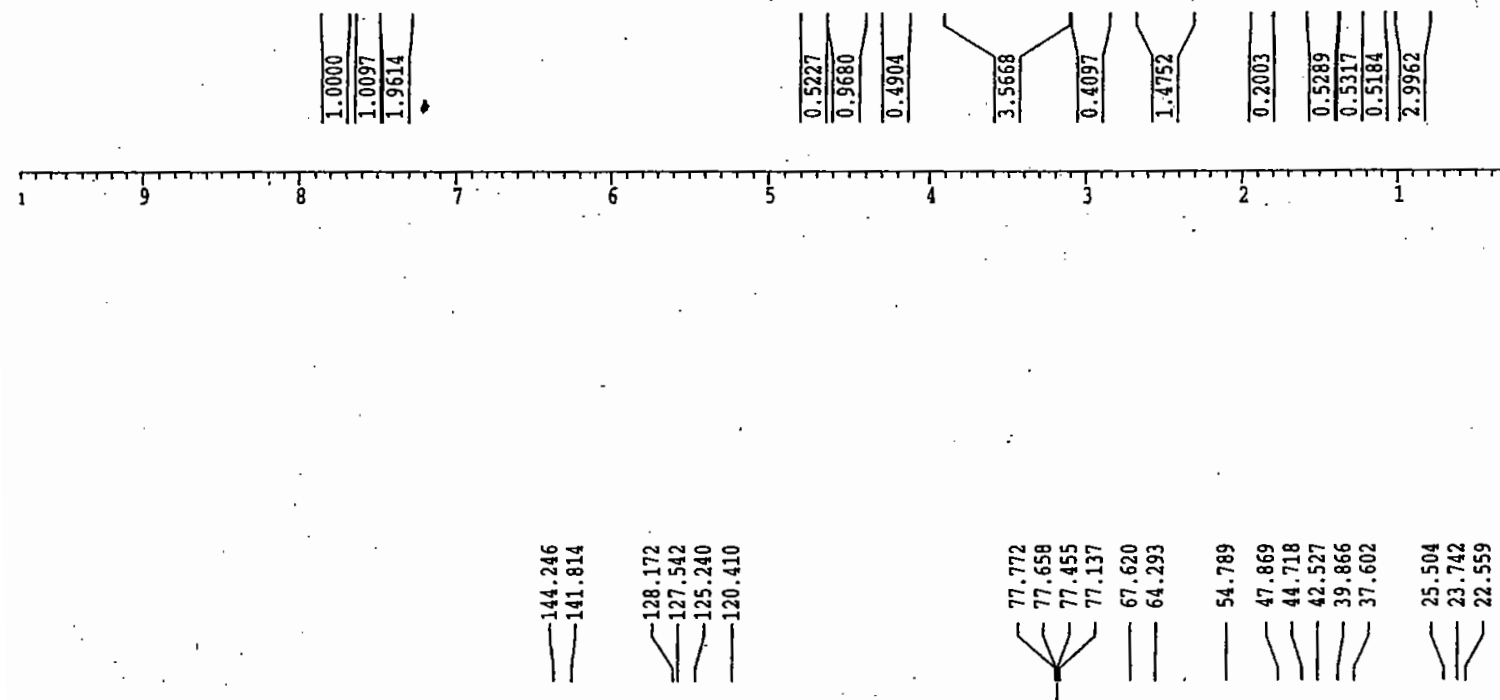


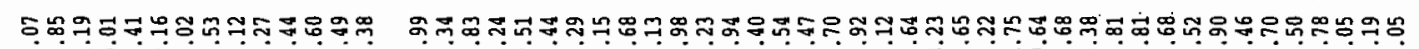

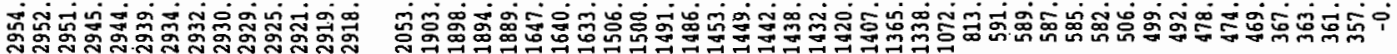
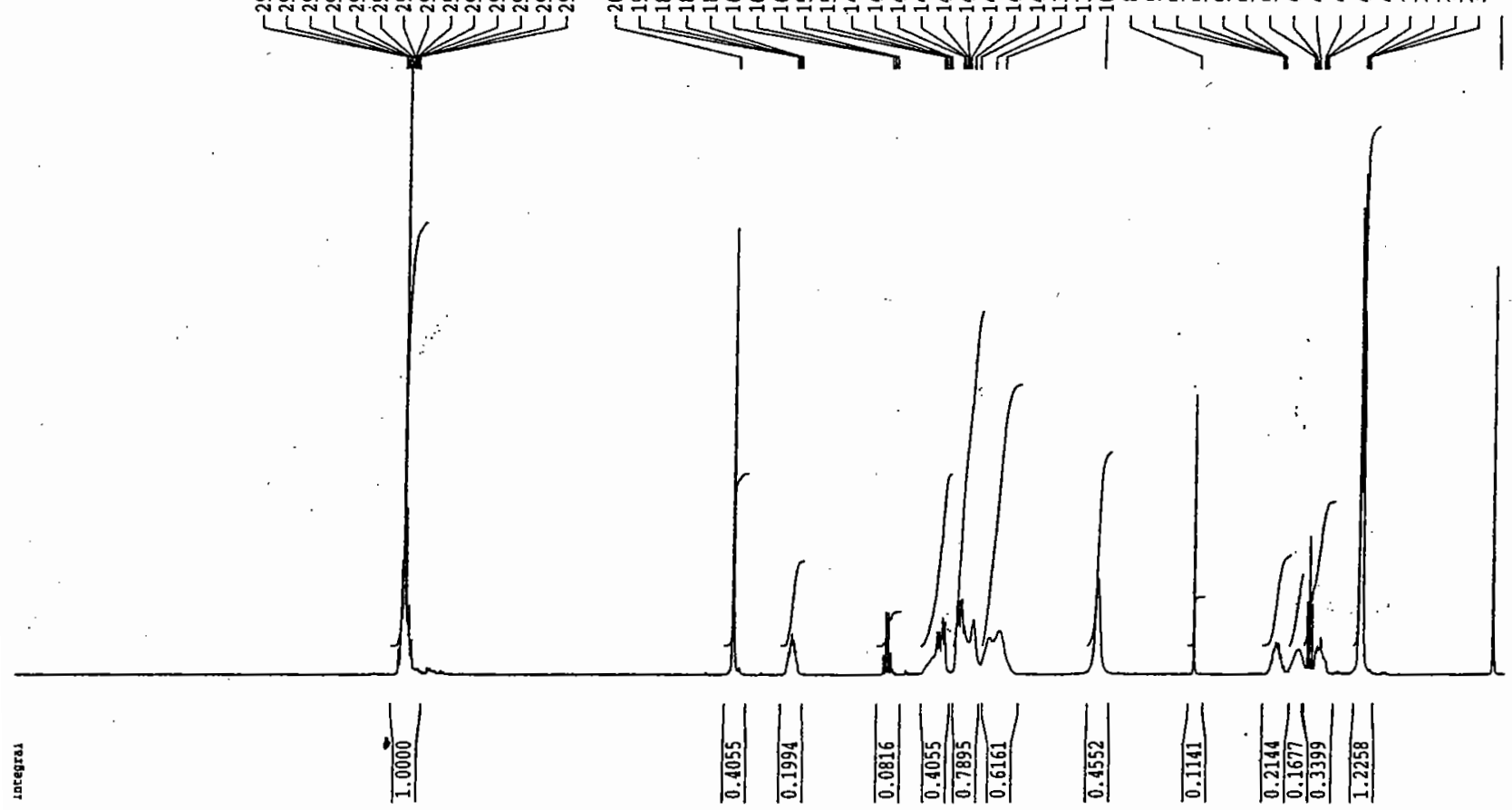

$\left|\begin{array}{c}\mid \\ 0 \\ 0 \\ 0 \\ 0\end{array}\right|$

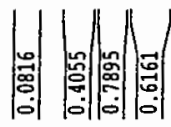

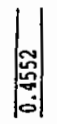

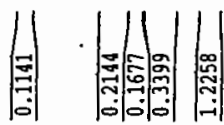

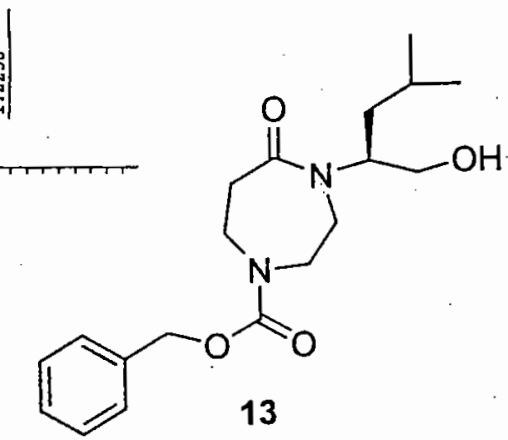

言

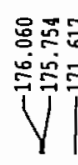

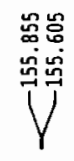

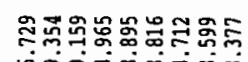

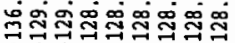

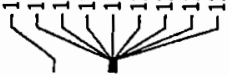

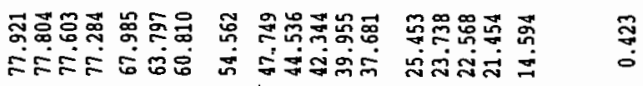

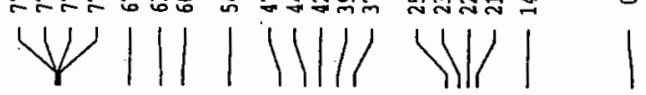



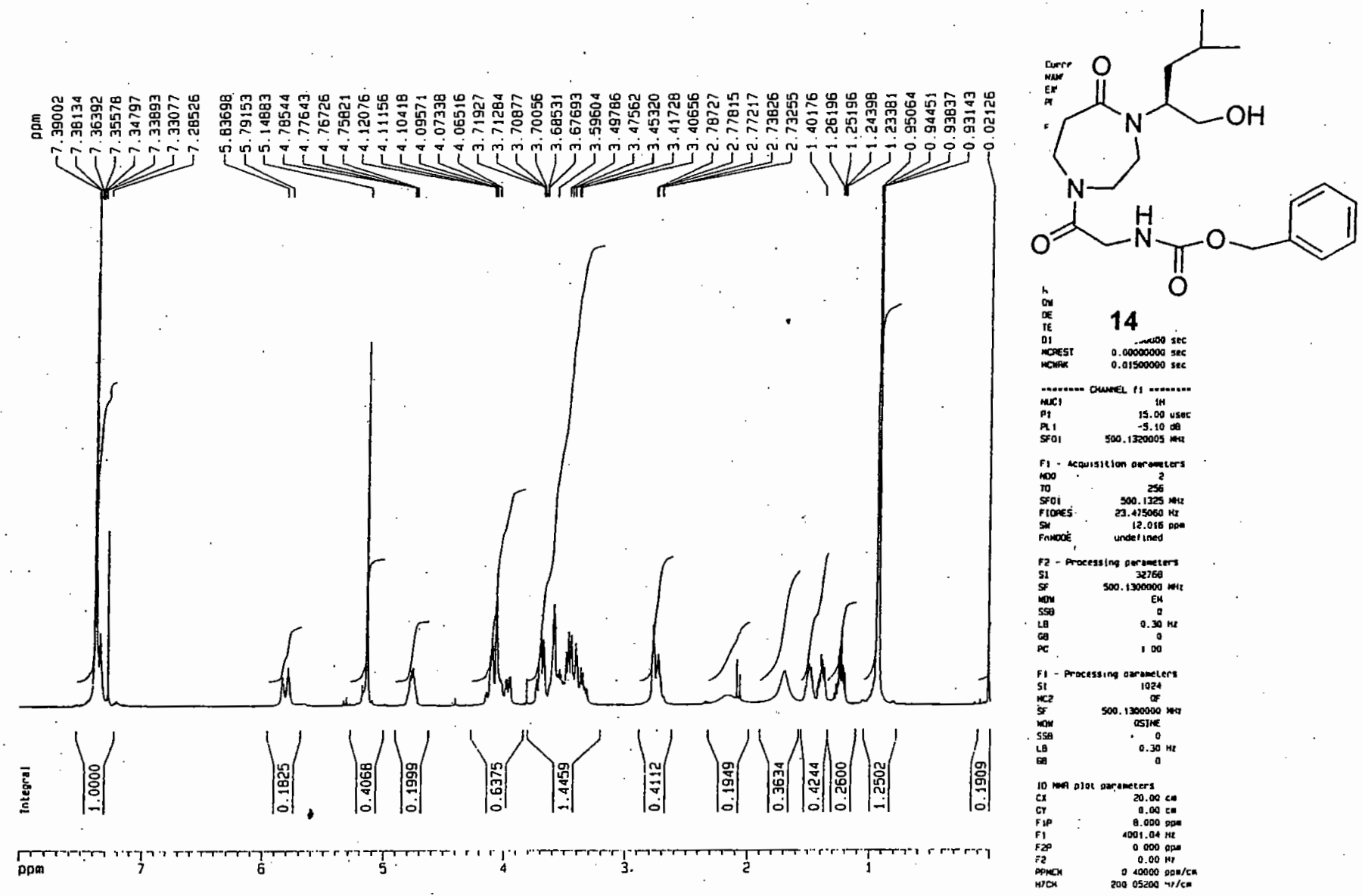

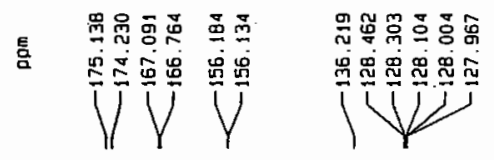

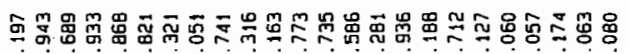

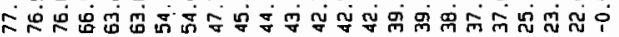

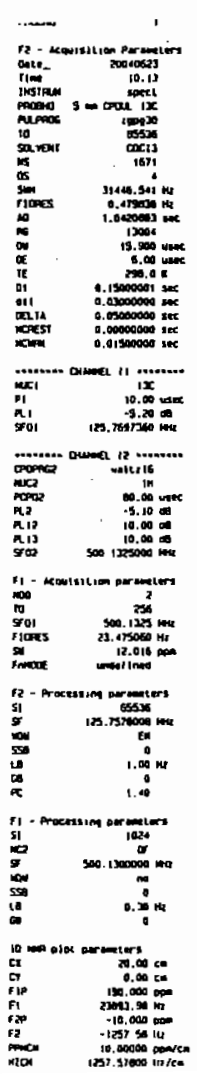




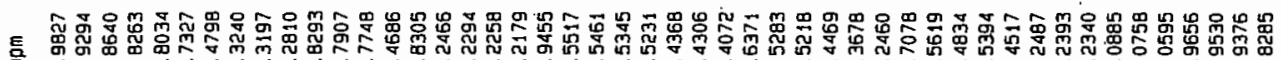
舟

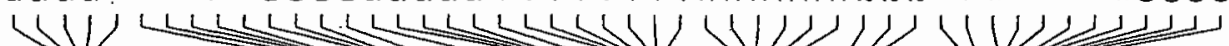
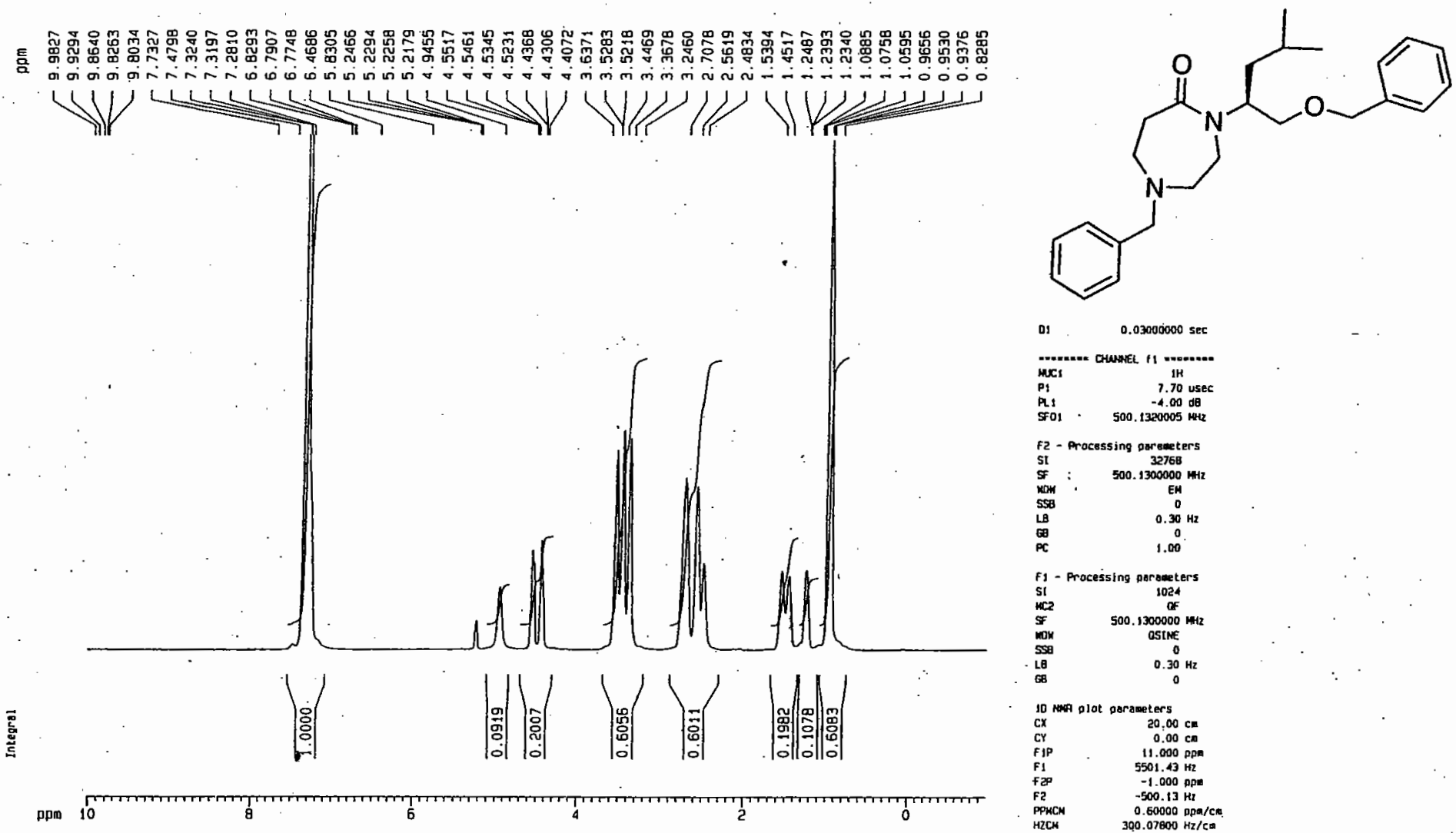

镸

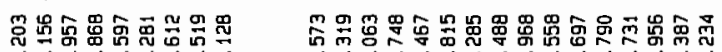

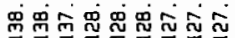

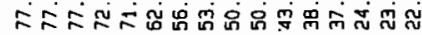

HWJW/H

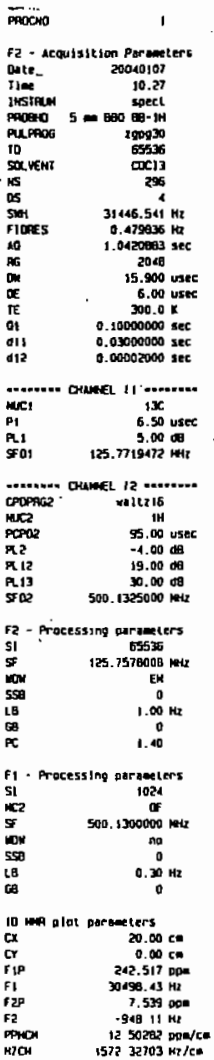



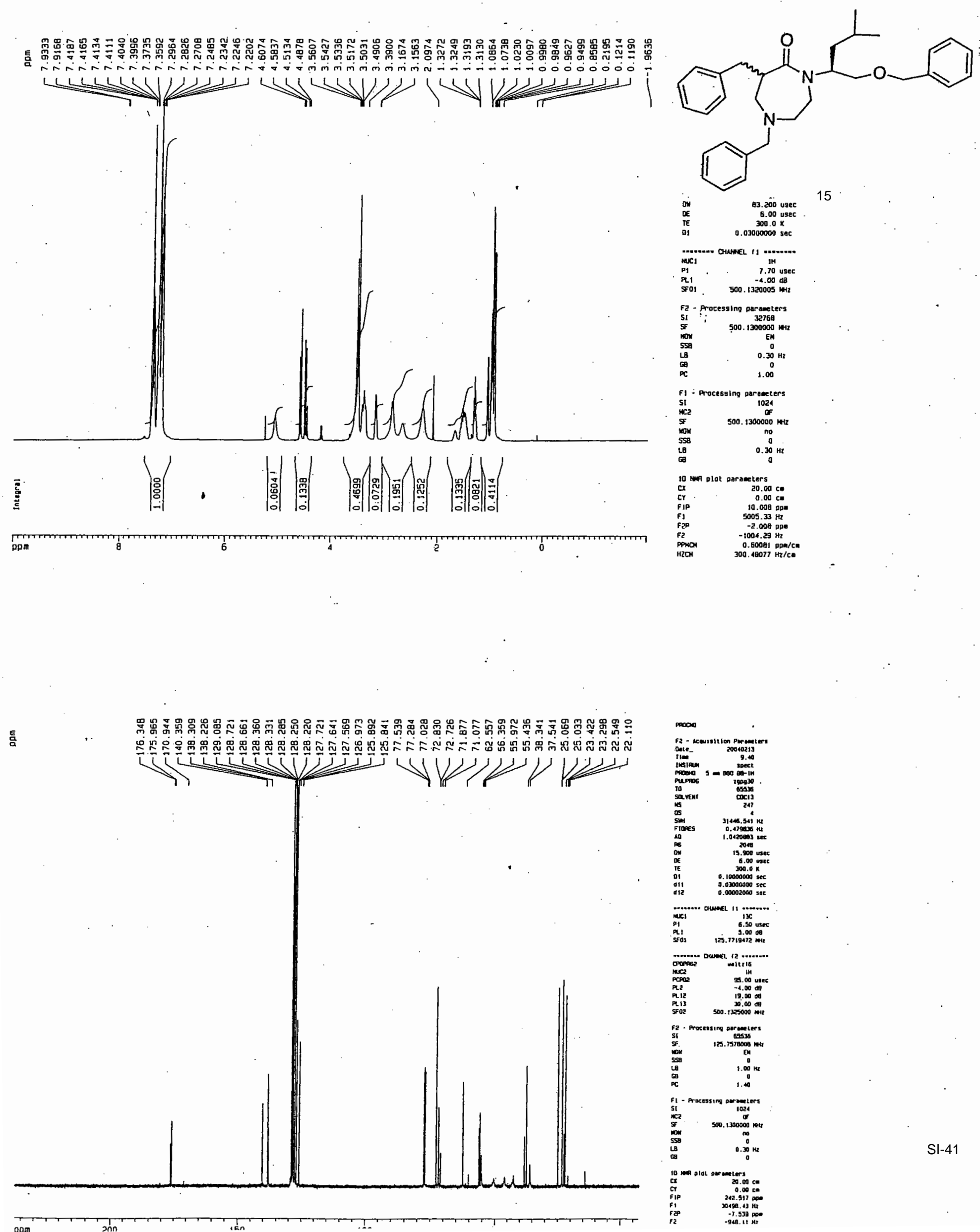

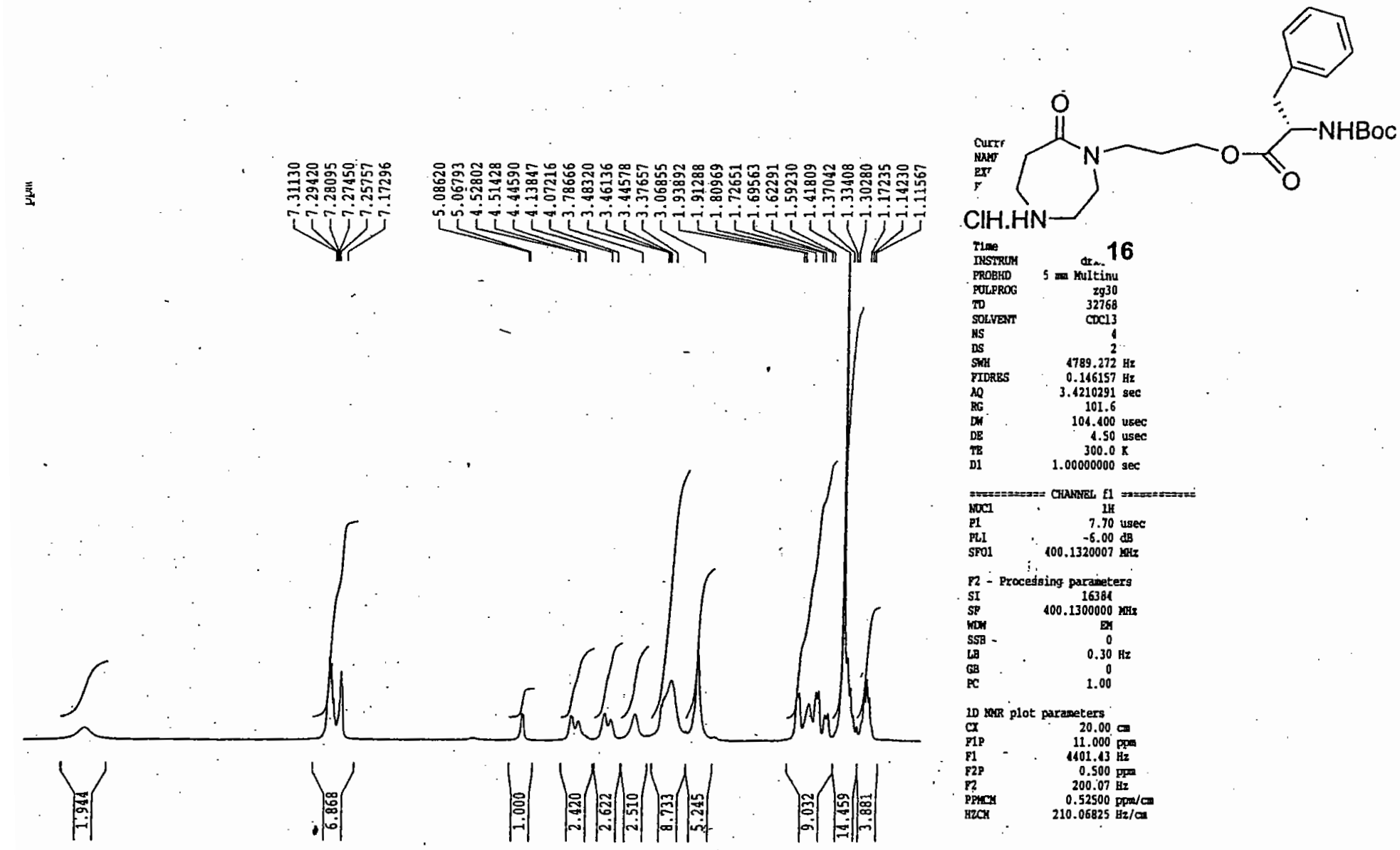

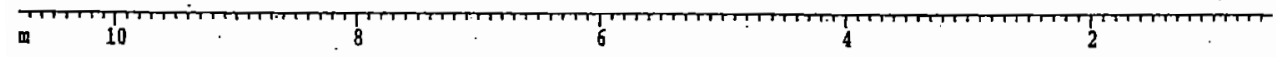

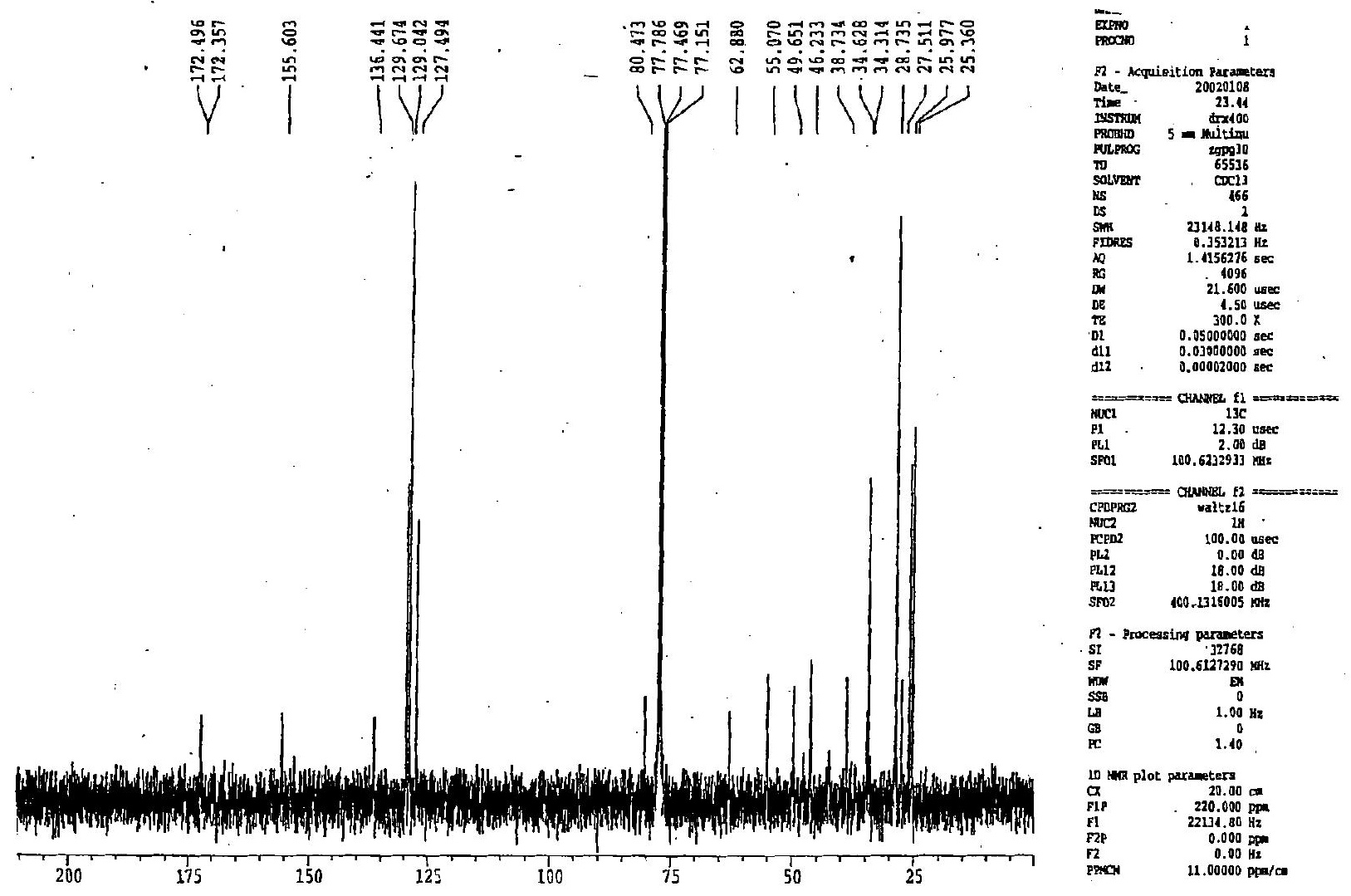

